\title{
Natural calcareous Norway spruce woodlands in Slovakia and their syntaxonomical classification
}

\author{
Peter Kučera1 ${ }^{1}$
}

Key words: Athyrio-Piceetalia, calcareous woodlands, forest plant communities, nomenclature, Picea abies, phytocoenology, supramontane woodland, syntaxonomy, Vaccinio-Piceetea, Western Carpathians.

Ključne besede: Athyrio-Piceetalia, gozdovi na karbonatih, gozdne rastlinske združbe, nomenklatura, Picea abies, fitocenologija, supramontanski gozdovi, sintaksonomija, Vaccinio-Piceetea, Zahodni Karpati.

Corresponding author:

Peter Kučera

E-mail: peter.kucera@uniba.sk

Received: 31. 3. 2021

Accepted: 13. 10. 2021

\begin{abstract}
A second version of the syntaxonomical classification of calcareous Norway spruce communities is presented for the region of Slovak Western Carpathians. Recent knowledge on delimitation of natural Norway spruce woodlands in Slovakia is summarized as well. As result, four in Slovakia traditionally recognized associations are distinguished: Seslerio caeruleae-Piceetum on the ecologically most extreme habitats, followed by Cirsio erisithalis-Piceetum (a replacement for pseudonymically used Cortuso matthioli-Piceetum), tall-forb community of Adenostylo alliariae-Piceetum and ca. species-poor low-forb community of Mnio spinosi-Piceetum (syn. Oxalido-Piceetum). Additionally, two new associations are differentiated: Fragario vescae-Piceetum ass. prov. standing between Cirsio-Piceetum and Adenostylo-Piceetum and acidified Hieracio murorum-Piceetum on deeper soils developed over rocks of the Mráznica formation. For nomenclatural reasons, new order Cortuso-Piceetalia is described for species-rich calcicolous communities of the class Vaccinio-Piceetea as well as subordinated new alliance Cortuso matthioli-Piceion for the supramontane calcicolous Norway spruce communities.
\end{abstract}

Izvleček

V članku predstavljamo drugo verzijo sintaksonomske klasifikacije smrekovih združb na karbonatu na območju Zahodnih Karpatov Slovaške, povzeli pa smo tudi trenutno poznavanje razmejitve naravnih smrekovih gozdov na Slovaškem. Rezultat so štiri tradicionalno prepoznane asociacije: Seslerio caeruleae-Piceetum na ekološko najbolj ekstremnih rastiščih, sledi Cirsio erisithalis-Piceetum (ki nadomešča psevdonim Cortuso matthioli-Piceetum), Adenostylo alliariae-Piceetum in vrstno siromašna asociacija z nizkimi zelišči Mnio spinosi-Piceetum (sin. Oxalido-Piceetum). Dodatno smo ločili dve novi asociaciji: Fragario vescae-Piceetum ass. prov., ki predstvalja vmesno asociacijo med Cirsio-Piceetum in Adenostylo-Piceetum, ter zakisano asociacijo Hieracio murorum-Piceetum na globljih tleh, razvitih na skalah formacije Mráznica. Zaradi nomenklaturnih razlogov smo opisali nov red Cortuso-Piceetalia za vrstno bogate združbe na karbonatih znotraj razreda Vaccinio-Piceetea in znotraj reda novo zvezo Cortuso matthioli-Piceion, kamor uvrščamo supramontanske smrekove združbe na karbonatih. 


\section{Introduction}

Syntaxonomical classification of the Norway spruce communities has undergone a long way since the establishment of the class Vaccinio-Piceetea by Braun-Blanquet et al. (1939). Hadač (1962) published a groundbreaking proposal for a fundamental division of this class based on floristical and ecological differentiation valid on supraregional to continental scale: separation of (1) usually species-poor communities growing on (very) strongly acidic soils, i.e. distributed over non-carbonate rocks and (2) species-rich communities growing on moderately to slightly acidic and neutral soils, i.e. inhabiting areas of various carbonate rocks. For these basic units the rank of order was assigned: the order Myrtillo-Piceetalia Hadač 1962 for the first group (the correct order name is Piceetalia abietis Pawłowski ex Pawłowski et al. 1928) and the order Athyrio-Piceetalia Hadač 1962 for the second group (for nomenclatural discussion see Kučera, in red.).

Acceptance of this syntaxonomical division is continuously growing in the European countries (for detail see Kučera, in red.), and even if authors did not recognize the order Athyrio-Piceetalia Hadac 1962 as a separate unit of the rank of order, their syntaxonomical system reproduced the respective two-component fundamental division (cf. Exner et al., 2002; Exner, 2007).

For the territory of Slovakia, two syntaxa checklists of the class Vaccinio-Piceetea Br.-Bl. in Br.-Bl. et al. 1939 were published up to the present: (1) Šomšák's list of syntaxa (in Mucina et al., 1985) based mostly on studies of Hadač et al (1969), Šoltés (1976) and Fajmonová (1978), and (2) recent derived checklist of Jarolímek et al. (2008a). Altogether five associations of natural calcicolous Norway spruce communities were traditionally differentiated: Seslerio-Piceetum, Cortuso-Piceetum, Adenostylo-Piceetum within the alliance Chrysanthemo-Piceion auct. non (Krajina 1933) Březina et Hadač in Hadač 1962 as well as Mnio spinosi-Piceetum, Oxalido-Piceetum classified within Oxalido-Piceion auct. non (Krajina 1933) Březina et Hadač in Hadač 1962 (cf. Kučera, 2012a; Kučera, in red.).

Kučera (2012a) published the first comprehensive syntaxonomical revision of the natural mountain Norway spruce syntaxa within Slovakia. An important part was a commented overview of distribution of the Norway spruce woodlands in the territory of the Western Carpathians, associated with a revision of traditional views on their natural distribution.

Aim of this paper is present an updated syntaxonomical classification of natural calcareous Norway spruce communities found in the territory of the Western Carpathians in Slovakia. The preceding syntaxonomical revisions were dedicated to syntaxonomy of Arolla pine communi- ties (Kučera, 2017; Kučera, 2019b) and coniferous wet woodland communities (Kučera, 2019a, with exception of bog woodland communities).

\section{Methods}

This contribution presents partial results of the syntaxonomical revision of the communities of the class VaccinioPiceetea Br.-Bl. in Br.-Bl. et al. 1939 present in Slovakia, originally intended to be part of the specialized volume of the Rastlinné spoločenstvá Slovenska (1995-) on the forest and scrub vegetation (Valachovič et al., msc.). Therefore the presented relevé dataset selection follows the methodical directions established for that monograph:

(1) The dataset for Slovak forest and scrub phytocoenoses was officially closed to the date 27. 5. 2016 and no newly published relevés were accepted. This regulation was here followed therefore this is the reason why for example numerous relevé data of Arolla pine woodlands published by Zięba et al. (2018) were not included in the statistical comparisons of the Vaccinio-Piceetea alliances. The final dataset was exported from Slovak vegetation database (CDF) (see Šibík, 2012; https://www.givd.info/ ID/EU-SK-001) by J. Šibík (Institute of Botany SAS, Bratislava) and made available to the respective authors of chapters on Slovakian forest and scrub vegetation.

(2) For forest communities only relevés with plot sizes $200-650 \mathrm{~m}^{2}$ were accepted. This regulation was also strictly followed even if that meant loss of some typical relevés with sizes between 100-200 $\mathrm{m}^{2}$ (for example Hadač et al., 1969, p. 272 etc. $^{1}{ }^{1}$.

(3) Selected taxa were merged to species aggregates such as Dryopteris carthusiana agg., Senecio nemorensis agg. (cf. Valachovič et al., msc.) or to the nearest mutual taxonomical rank (e.g. Solidago virgaurea ssp. minuta + Solidago virgaurea).

The resulting dataset was stored in Turboveg for Windows database software (Hennekens c1998-2020) (cf. Hennekens \& Schaminée, 2001) and selection of Vaccinio-Piceetea phytocoenoses with respect of above-mentioned limitations was performed by the author. Some of the relevés were revised according to originally published data.

In respect of the thorough revision of literature sources, records of explicit successional stages (e.g. some relevés of Krajina (1933)) as well as relevés with missing ground layer species were excluded. Relevés of phytocoenoses with natural occurrence of Pinus cembra were also excluded because they are classified within the alliances Calamagrostio

1 However, the excluded relevés were considered within the proposal of syntaxonomical classification within the respective associations. 
variae-Pinion cembrae and Homogyno alpinae-Pinion cembrae (cf. Kučera, 2017). With regard to different floristical patterns of calcareous and acid Norway spruce woodlands (cf. Kučera, 2012a), the set of calcicolous phytocoenoses was separated.

In attempt to maximize quality of data selected for statistical classification of Slovak Picea abies phytocoenoses of the Western Carpathians, the available relevé data were thoroughly re-analysed with regard to revisions of the natural distribution of Picea abies communities in Slovakia (see below). The most important part is to eliminate potential data contamination with floristical and ecological influence of anthropogenically degraded forest communities, especially exclusion of data recorded in unequivocally identified substitutionary Norway spruce stands (see below in the Results, for the detailed explanation see Kučera (2012a)).

As result, a strict restriction of altitudinal limit was adopted: relevé data from localities below the elevation $1400 \mathrm{~m}$ a.s.l. were excluded. This subjective decision is based on the field observations of Fagus and mixed Fagus woodland stands found above $1300 \mathrm{~m}$ a.s.l. on expositions of all four cardinal directions, with emphasis on the preserved Fagus stands reaching with their artificially lowered upper forest line (!) above 1340-1350 m a.s.l. on non-carbonate bedrock. In the carbonate regions, such artificial upper forest line of mixed Fagus woodlands of the class Carpino-Fagetea was confirmed in the field above 1360-1390 $\mathrm{m}$ a.s.1.

Below the chosen altitudinal limit (1400 m a.s.l.) only carefully selected relevés of Picea abies phytocoenoses were accepted into the dataset. They represent plant communities mostly found on ecologically more extreme habitats which hinder development of the Carpino-Fagetea communities, within the Western Carpathians for example on considerably steep and rocky habitats with shallow soils which support an extragradal occurrences of natural calcareous Norway spruce phytocoenoses (cf. Fajmonová, 1978).

No other phytocoenological relevés were eliminated, even if they represented untypical of problematically identifiable phytocoenoses. The complete list of the used relevés is summarily presented by Kučera (in prep.), see also below the particular associations. ${ }^{2}$

The final dataset with 80 relevés was exported to the software package JUICE (Tichý c1998-2020) (cf. Tichý, 2002) for further modifications (see above) and, subsequently, exported for statistical analysis performed by the

2 In comparison to the dataset used for calcicolous Norway spruce communities by Kučera (2019a), tab. 1: “76 relevés"), four relevés were added: Kobzáková (1987), tab. 8, rel. 7; Miadok (1995), p. 60, rel. 2; Kučera (2012a), p. 289, rels. 9-10. software package SYN-TAX 2000 (Podani, 2001a). The ordinal hierarchical clustering was executed to evaluate also quantitative information provided by ordinal BraunBlanquet's scale (cf. Kučera, 2011a) and the Podani's discordance coefficient was used as it takes into account also presence vs. absence relation (Podani, 2001b). For confrontation also the ordinal nonhierarchical clusterings were performed using the Podani's discordance coefficient. Further classification comparisons and verifications included variants of the ordinal hierarchical/nonhierarchical clustering without the most species-rich communities ([1] Seslerio-Piceetum or [2] Seslerio-Piceetum and Cirsio-Piceetum).

The final relevé classification is based on classification of data on the field layer $\left(E_{1}\right)$ and the ground layer $\left(E_{0}\right)$ only, because tree species data deformed the statistical results and for example Sorbus aucuparia-pseudocommunities were created without having other sufficient diagnostic floristical attributes. The resulting dendrogram is presented in the Figure 1.

The differential attributes of the respective syntaxa (fidelity and frequency values) and resulting tables were elaborated within JUICE; the concept of fidelity was used (Chytrý et al., 2002a). Fidelity calculations ( $\varphi$-values) are based on the presence/absence data with a standardization of relevé groups to an equal size. Performing the Fisher's exact test, zero fidelity was given to species with significance $\mathrm{P}>0.05$ in a particular cluster (Tichý \& Chytrý, 2006).

The formal sequence of species groups in the Table 2 is adjusted according to the template of differential tables of the Vegetation of the Czech Republic, Vol. 4 (Chytrý et al., 2013a), i.e. trees, shrubs, differential and other species of the field and ground layer separately; constancy and fidelity values equal and higher than " 50 " are highlighted in boldface type. Statistically determined diagnostic species are ranked according to fidelity values. The conventional levels of statistical significance $(0.05,0.01$ and 0.001 , Fisher's exact test) for the particular species are indicated with asterisks $(*, * *, * * *)$. Due to rather low total number of available natural Norway spruce relevés, species with the lowest diagnostic value (Fisher's exact test 0.05 -limit) were also retained as "diagnostic species" for individual syntaxa, bearing in mind their availability for future comparisons and potential use during field research.

However, in the text are given in number reduced groups of the recommended diagnostic species (highlighted in grey in the Table 2) to limit the respective species lists, recruited from species which (1) usually have $\varphi$-value $\geq 0.50$ or (2) are concentrated in the respective community. Non-highlighted statistically defined diagnostic species could also be used for delimitation of the respective 


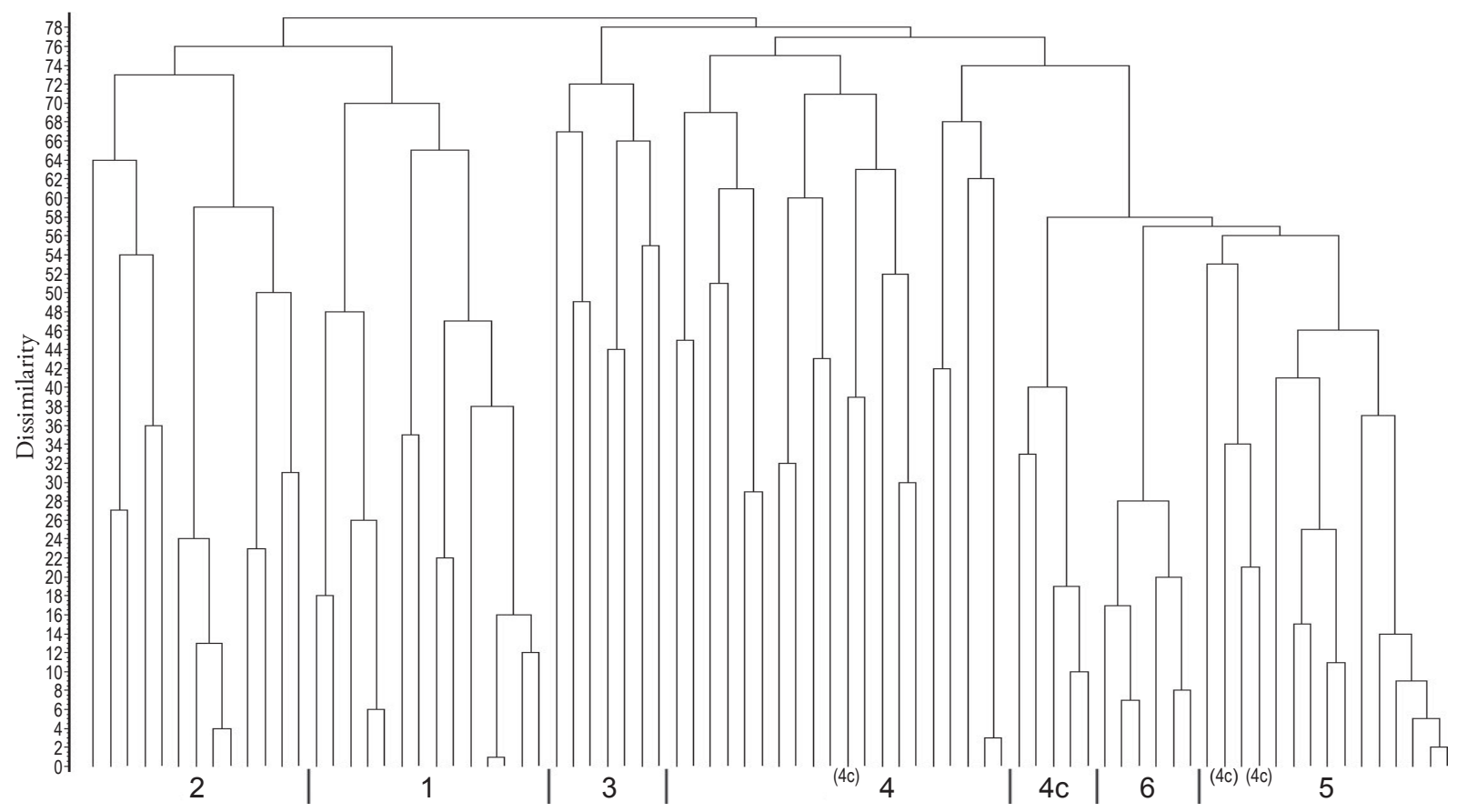

1 - Seslerio caerulae-Piceetum; 2 - Cirsio erisithalis-Piceetum, 3 - Fragario vescae-Piceetum ass. prov., 4 -Adenostylo alliariae-Piceetum, 4c - Adenostylo-Piceetum stellarietosum nemorum, 5 - Mnio spinosi-Piceetum, 6 - Hieracio murorum-Piceetum

Figure 1: Dendrogram of the ordinal hierarchical clustering of the relevés of natural calcareous Picea abies communities from Slovakia. Slika 1: Dendrogram ordinalne hierarhične klasifikacije popisov naravnih združb z vrsto Picea abies na karbonatu s Slovaške.

community, but only with careful consideration of their association with other species in other communities and also with consideration of their cover-abundance values in the evaluated and other communities: e.g. Adenostyles alliariae is frequent species in the whole group of natural calcicolous Picea abies woodlands, however, in the association Adenostylo alliariae-Piceetum it is (co-)dominant species with very high frequency.

Division of the diagnostic species into differential and Braun-Blanquetian character species is not applied, because (1) this statistical evaluation is based only on limited dataset, (2) character species (for example for Slovak vegetation) are frequently traditionally subjectively assigned to a preselected syntaxa, even in the national monographs, and thus do not rely on statistical determination (for example Adenostyles alliariae).

Species taxa names from the Western Carpathian relevés follow checklists of Marhold et al. (1998), Kubinská \& Janovicová (1998) and Pišút et al. (1998), exceptions are for example the names of Sesleria caerulea (L.) Ard. (cf. Foggi et al., 2001) and Soldanella marmarossiensis agg. (S. hungarica auct. slov., cf. Valachovič et al., 2019), or other species adapted from the particularly cited studies. Nomenclatural evaluation of the discussed syntaxa names is regulated according to the $4^{\text {th }}$ edition of the Code
(Theurillat et al., 2021), highlighted are the cited Code Articles ("Art.") and Recommendations ("Rec."). The names of the classes Vaccinio-Piceetea Br.-Bl. in Br.-Bl. et al. 1939 and Carpino-Fagetea Jakucs ex Passarge 1968 are hereafter given without author citations. Common soil names (within the associations'descriptions) are supplemented with the 2006 edition of the World Reference Base for Soil Resources names given according to the reference table in the Societas pedologica Slovaca (2014).

The sites of the analysed relevés within the individual associations in Slovakia are given in the Figures 2 and 3. The coordinates of the dataset relevés taken from the CDF were partially revised, especially those created for older surveys without pre-existing coordinate data. The maps were created using the Free and Open Source QGIS (QGIS.org, 2021), the base layer (terrain) is provided by Geodesy, Cartography and Cadastre Authority of the Slovak Republic (see https://zbgis.skgeodesy.sk).

Extended characterization of the discussed associations - their distribution within the Western Carpathians, ecology, dynamics of the stand, notes to nature conservation - is summarized by Kučera (in prep.), in this paper are mainly discussed: floristical differentiation, syntaxonomical variability as well as important nomenclatural and syntaxonomical problems. 


\section{Results and discussion}

\section{General delimitation of the considered plant communities}

Regionally developed intrazonal coniferous orobiomes (i.e. within the nemoral zone) of the main Central European mountain ranges are analogous counterparts of the taiga (zono)biom of the northern Eurasian latitudes. The most frequent and usually the exclusive component of these orobiomes is a Picea abies mountain woodland, only the most highest mountain ranges (Alps, Tatra Mountains etc.) possess (mixed) Arolla pine woodlands.

Numerous Picea abies plant communities were historically syntaxonomically included into the class VaccinioPiceetea. Due to the impacts of pre-/historical land management, general patterns of the distribution of Norway spruce stands in the Western Carpathians have considerably changed. Fully in agreement with Zlatník (1959, p. 20) I am of the opinion that the key to understand the original [primary] overall composition of forests in Slovakia is a reconstruction of the natural distribution of Fagus sylvatica and its proportional participation on composition of these forests.

This approach requires determination of potential natural distribution of Fagus sylvatica or, more precisely, determination of horizontal and vertical potential natural distribution of woodlands (co-)dominated by F. sylvati$c a$ as well as mixed deciduous-coniferous woodlands of higher montane altitudes with still considerable ecological influence of $F$. sylvatica on the plant species composition of the field layer. The Zlatník's concept of forest vegetation reconstruction (Zlatník, 1957; Zlatník, 1959; Zlatník, 1975; Zlatník, 1976; Zlatník, 1978) is similar to geobotanical reconstruction approaches (cf. Tüxen (1956) and later updates and variations, cf. Neuhäusl (1994)). Moreover in regard to the recent climate change, specialized attention should be paid to the period up to 1950-1980, i.e. before the notable recent anthropogenic impact on climate variables important for the development of woodland orobiomes.

In the territory of the Western Carpathians within Slovakia, planted Norway spruce stands are frequently easily recognizable because they abruptly border to (semi-)natural and more resilient beech or beech-silver fir stands of the class Carpino-Fagetea even within the same slope of a valley, i.e. at the originally same habitat. Such plantations have usually limited duration, however, existence of several generations of uninterrupted Norway spruce monocultures is known in some regions (Šimurdová, 2001) or Norway spruce monocultures could have re- placed already historically changed forest vegetation (see Nižnanská, 1983). In such cases, centuries long cultivation of Norway spruce stands gradually effects regional species composition and also habitat conditions.

All such plantations represent less or more anthropogenically influenced stages of original plant communities of the class Carpino-Fagetea and they should be classified within that class (cf. Hadač \& Sofron (1980) and corrections by Kučera (2012a, chap. 5.27); see also Zlatník (1975, p. 83-84, 86).

The higher up into the mountains, the harder becomes the differentiation between the native and anthropogenic Norway spruce stands replacing the original mixed European beech woodland. However, in the Slovakian Western Carpathians the beech-fir woodlands are sufficiently represented even in the altitudes $1000-1200 \mathrm{~m}$ a.s.l. therefore the Norway spruce cultures as temporary coniferous stages of Carpino-Fagetea communities are mostly reliably identifiable.

\section{General lower line of Norway spruce altitudinal vegetation zone in the Western Carpathians}

Throughout the previous decades several variants of the elevational span of the Norway spruce altitudinal vegetation zone were published for the Western Carpathians (see Kučera 2012a, chap. 3): according to various authors, altitudes of the general lower limit of this zone were estimated to different values within the range from (1000) 1100 up to 1250 (1300) m a.s.l., the natural general (i.e. climatic) upper limit was assumed to be situated also at different values ranging from 1300 to 1550 (1600) m a.s.l.

Inconsistency of the referred assessments is evident. Evaluations of the assumed natural altitudinal distribution of Norway spruce woodland within the majority of previous vegetational outlines published for the territory of Slovakia, either from the view of botany (Holub \& Jirásek, 1967; Futák, 1972; Michalko \& Berta, 1972; Michalko et al., 1980; Michalko et al., 1986; Šomšák, 1998; cf. also Medwecka-Kornaś, 1972) or forest typology (Hančinský, 1972; Hančinský, 1977; Randuška, 1986; Fleischer \& Chmiel, 2010; cf. also Mindáš, 1999) and geography (Plesník, 1961; Plesník, 1995; Plesník, 2004) are at least partly unsatisfying (for details see Kučera (2012a)). Debatable geobotanical conclusions of the cited studies and other works were also reflected in specialized manuals for nature conservation (Stanová et al., 2002; Viceníková et al., 2003; and previous versions).

The most probable explanation of inconsistencies between the previously published results and the recently 
revised field situation is that insufficient attention was paid to identification of anthropogenic, secondary Norway spruce stands in the field, especially within the highest part of the montane altitudinal zone, and to their differentiation from natural Norway spruce woodlands. Formation of the former group is frequently connected with the long-term spontaneous succession of Picea abies on formerly deforested areas once used for high mountain grazing or even mowing in some localities (subanthropogenic origin of Picea stands; Kučera, 2012a).

In the various Western Carpathian mountain ranges, occurrences of (mixed) upper montane Fagus woodlands over the traditionally most accepted general altitudinal limit $1250 \mathrm{~m}$ a.s.l. for existence of European beech woodland were confirmed along with a widespread gradual recovery of such forests by the processes of secondary succession of $F$. sylvatica within anthropogenic $P$. abies stands (Kučera, 2011b; Kučera, 2012a; Kučera, 2013a; Kučera, 2014a; Kučera, 2015a; and other until now unpublished sequels in this topic).

Therefore the current general delimitation of the natural distribution of communities of the class Vaccinio-Piceetea is based on detailed revision of the occurrences of more or less natural (mixed) Fagus woodlands in the field, especially those which existed before 1950s. As such they were recorded in the historical orthophoto map of Slovakia with the oldest aerial photos dated to the year 1949 (Historická ortofotomapa Slovenska s. d.).

Considerably revised was also distribution of so-called Fagus-free coniferous woodlands in the middle and lower montane altitudes of Slovak basins and mountain ranges which were also traditionally classified as plant communities of the class Vaccinio-Piceetea (e.g. Kučera, 2012b; Kučera, 2012c; Kučera, 2014b; Kučera, 2015b; Kučera et al., 2009).

The above-cited results of the field revision of naturalness of Norway spruce woodland communities in the Western Carpathians signify that the general lower limit of Norway spruce altitudinal vegetation zone (in Latin "gradus" as an altitudinal counterpart to a latitudinal vegetation zone [Latin "zona"], cf. Holub \& Jirásek (1967, p. 79-8) and Krippel (1986, p. 260)) should be placed to considerably higher altitudes than traditionally accepted. In Slovakia, this altitudinal limit most probably has not naturally descended below $1350 \mathrm{~m}$ a.s.l. at all. In respect to various expositions and mountain ranges, the estimated range is probably within the interval between ca. (1370) 1380-1430 (1450) m a.s.l. It should be emphasized that the respective estimation is projected for $20^{\text {th }}$ century before the era of gradually more visible impacts of the anthropogenic climate change (i.e. before ca. 1990, cf. Lapin et al., 2005; Faško et al., 2008). In addition, important though insufficiently known is the original natural elevational span of the transitional belt between unequivocally classifiable Carpino-Fagetea and Vaccinio--Piceetea communities.

The extragradal natural occurrences of Norway spruce communities in lower altitudes are, obviously, not counted to that range because the respective communities originated in terrain-induced ecologically extreme habitats inhibiting the development of so-called climax woodlands forming the predominating woodland vegetation (cf. also Zlatník, 1975, p. 103-104; Zlatník, 1978, p. 327-328). Such ecologically peculiar Norway spruce communities are altitudinally lying in the uppermost part of the montane belt occupied by communities of the class Carpino-Fagetea. Considering the natural calcareous Norway spruce woodland types, the most frequent examples of such extragradal communities are phytocoenoses of the asociation Seslerio-Piceetum Fajmonová 1978 developed at altitudes below ca. 1370-1400 m a.s.l.

A detailed review of Norway spruce woodlands documented in the individual mountain ranges of the Western Carpathians as well as comments to communities incorrectly classified within the class Vaccinio-Piceetea was already published (see Kučera, 2012a). A shortened catalogue of records of the plant communities which should not be considered as natural calcicolous Norway spruce communities is given in the Table 1 . As such they should be classified as anthropogenic degraded variants of plant communities of the class Carpino-Fagetea, mostly within the order Cephalanthero damasonii-Fagetalia sylvaticae Boeuf et Royer in Boeuf et al. 2014, ${ }^{3}$ uniting the European calcareous Fagus and Fagus-Abies woodlands, i.e. communities with ecological conditions and species composition determined by special ecological regime provided by mutual effect of base-rich soils (mostly various calcareous soils: rendzic leptosols, rendzinas, pararendzinas) and distinctive landscape relief usually developed in mountain regions, formed by various sedimentary calcareous rocks (limestones, dolomites etc.). Some of the considered secondary Norway spruce communities also might belong to the group of mesotrophic (silicicolous to semicalcicolous) woodlands united into the order Fagetalia sylvaticae Tüxen 19314,5 (cf. Boeuf et al., 2014).

3 Cf. the proposal of classification of (mixed) Fagus woodlands within the class Carpino-Fagetea into three cardinal units by Boeuf et al. (2014).

4 Willner (in Willner et al., 2015) argued for validity of the order name Fagetalia sylvaticae Pawłowski 1928. Despite the all argumentation, Pawłowski (1928, p. 15) did not provide a real unambiguous direct or indirect reference to an earlier effectively published sufficient diagnosis (cf. Theurillat et al., 2021: Art. 2b). Scientific nomenclatural evaluations and decisions should be based on clearly stated information or data presented in the considered source; on the contrary, obscure guessing and wishing of a "proper reference" do not count among convenient scientific approaches. Therefore I identify with 
The catalogue given in the Table 1 demonstrate that anthropogenic calcicolous Norway spruce phytocoenoses were recorded in rather large number within the Western Carpathians. Probably most of them were used in the spe-

\footnotetext{
Theurilat's conclusion (in Willner et al., 2015, p. 177) that the name Fagetalia sylvaticae Pawłowski 1928 is the invalidly published name. The derived later name Fagetalia sylvaticae Pawłowski ex Pawłowski et al. 1928 is invalidly published as well (cf. Kučera, 2013b); the oldest validly published Fagetalia-name is Fagetalia sylvaticae Tüxen 1931 and it has already been recognized by Boeuf et al. (2014), see also Renaux et al. (2019).

5 This order name is based on the alliance Fagion septentrionale Tüxen 1931 nom. illeg. (Art. 34a) described for calcicolous Fagus sylvatica phytocoenoses of the Hildesheimer Wald-Ith region in northern Germany. Following the original diagnosis, the order is not characterized by distinctive calciphytes, however, the phytocoenotic composition of the respective association Fagetum subhercynicum Tüxen 1931 (Tüxen, 1931, p. 104 and the attached table) is clearly influenced by the calcareous geological substrate.
}

cialized statistical surveys on either diagnostic and other significant species (cf. Chytrý et al., 2002b; Jarolímek et al., 2008b; cf. also Jahn, 1985; Exner et al., 2002) or phytocoenological affinity of a chosen taxon (cf. Valachovič et al., 2019) - see the very high total number of relevés used in that studies in comparison to the here presented syntaxonomical revision. Therefore the respective results of those studies are misleading or incorrect when considering the class Vaccinio-Piceetea because they factually represent a mixture of relevé data of two classes: CarpinoFagetea and Vaccinio-Piceetea. In contrast to the statement of Chytrý et al. (2002b, p. 406) I am of the opinion that the number of secondary Norway spruce relevés (belonging to the class Carpino-Fagetea) used in that study (and similar other studies) represented a considerable part of evaluated relevé data.

Table 1: Catalogue of records of the anthropogenic calcicolous (mixed) Norway spruce phytocoenoses incorrectly classified within the class Vaccinio-Piceetea.

Tabela 1: Seznam literaturnih virov z antropogenimi smrekovimi združbami na karbonatnih (mešanih) substratih, nepravilno uvrščenih v razred Vaccinio-Piceeted.

"Only published studies are listed, for details on manuscripts' details see Kučera (2007, 2012a). Geomorphological units are given according to Kočický \& Ivanič (2011)."

Part 1. Slovakia

\begin{tabular}{|c|c|c|c|c|}
\hline Authors & $\begin{array}{l}\text { Geomorphological } \\
\text { unit }\end{array}$ & Original name of unit & $\begin{array}{l}\text { Proportion of Carpino- } \\
\text { Fagetea relevés }\end{array}$ & Remarks \\
\hline Klika (1926) & Vel'ká Fatra & Piceetum excelsae & All relevés & \\
\hline Klika (1929) & $\begin{array}{c}\text { Vel'ká Fatra, } \\
\text { Krivánska Fatra }\end{array}$ & $\begin{array}{l}\text { Typ borůvkový, Vaccinium } \\
\text { myrtillus-Calamagrostis varia }\end{array}$ & All relevés & \\
\hline Sillinger (1933) & $\begin{array}{l}\text { Dumbierske Tatry, } \\
\text { Královohol'ské Tatry }\end{array}$ & $\begin{array}{l}\text { Piceetum excelsae normale } \\
\text { calcicolum }\end{array}$ & All relevés & $\begin{array}{l}\text { Only synoptic table. Author's } \\
\text { original alternative name: } \\
\text { Piceeto-Abietetum normale } \\
\text { calcicolum Sillinger } 1933 .\end{array}$ \\
\hline Sillinger (1933) & $\begin{array}{l}\text { Ďumbierske Tatry, } \\
\text { Královohol'ské Tatry, } \\
\text { Slovenský raj }\end{array}$ & $\begin{array}{l}\text { Piceetum excelsae altherbosum } \\
\text { calcicolum }\end{array}$ & Pro parte maj. & Only synoptic table. \\
\hline Sillinger (1933) & $\begin{array}{l}\text { Dumbierske Tatry, } \\
\text { Královohol'ské Tatry, } \\
\text { Slovenský raj }\end{array}$ & $\begin{array}{l}\text { Piceetum excelsae myrtilletosum } \\
\text { calcicolum }\end{array}$ & Almost all relevés & Only synoptic table. \\
\hline Svoboda (1939) & Západné Tatry & Piceetum altherbosum & Pro parte & Only synoptic table. \\
\hline $\begin{array}{l}\text { Grebenščikov } \\
\text { et al. (1956) }\end{array}$ & Vel'ká Fatra & $\begin{array}{c}\text { Piceetum excelsae myrtilletosum } \\
\text { Klika }\end{array}$ & Page $117-118$ & \\
\hline $\begin{array}{l}\text { Grebenščikov } \\
\text { et al. (1956) }\end{array}$ & Vel'ká Fatra & $\begin{array}{c}\text { fragments of Norway spruce } \\
\text { woodland with Cortusa matthioli } \\
\text { and Luzula sylvatica }\end{array}$ & Page 119 & \\
\hline $\begin{array}{l}\text { Samek et al. } \\
(1957)\end{array}$ & $\begin{array}{l}\text { Vysoké Tatry, } \\
\text { Belianske Tatry }\end{array}$ & $\begin{array}{c}\text { Piceetum excelsae altherbosum/ } \\
\text { Adenostyleto-Piceetum }\end{array}$ & Pro parte & \\
\hline $\begin{array}{l}\text { Samek et al. } \\
\text { (1957) }\end{array}$ & Vysoké Tatry & Piceetum normale silicicolum & All relevés & $\begin{array}{c}\text { Phytocoenoses in fact } \\
\text { considerably influenced by } \\
\text { carbonate rocks. }\end{array}$ \\
\hline $\begin{array}{l}\text { Samek et al. } \\
(1957)\end{array}$ & $\begin{array}{c}\text { Vysoké Tatry } \\
\text { (? Belianske Tatry) }\end{array}$ & Piceetum normale calcicolum & ? Pro parte & \\
\hline
\end{tabular}




\begin{tabular}{|c|c|c|c|c|}
\hline Authors & $\begin{array}{l}\text { Geomorphological } \\
\text { unit }\end{array}$ & Original name of unit & $\begin{array}{l}\text { Proportion of Carpino- } \\
\text { Fagetea relevés }\end{array}$ & Remarks \\
\hline $\begin{array}{l}\text { Samek et al. } \\
(1957)\end{array}$ & $\begin{array}{c}\text { Vysoké Tatry } \\
\text { (? Belianske Tatry) }\end{array}$ & Abieto-Picetu $\quad \mathrm{m}$ & All relevés & \\
\hline $\begin{array}{l}\text { Zahradníková- } \\
\text { Rošetzká (1957) }\end{array}$ & Ďumbierske Tatry & $\begin{array}{l}\text { Piceetum excelsae normale } \\
\text { calcicolum Sillinger } 1933\end{array}$ & - & (Without relevés.) \\
\hline $\begin{array}{l}\text { Zahradníková- } \\
\text { Rošetzká (1957) }\end{array}$ & Ďumbierske Tatry & $\begin{array}{c}\text { Piceetum excelsae altherbosum } \\
\text { calcicolum Sillinger } 1933\end{array}$ & Pro parte min. & (Without relevés.) \\
\hline $\begin{array}{l}\text { Hadač et al. } \\
\text { (1969) }\end{array}$ & Belianske Tatry & $\begin{array}{c}\text { Dryoptero-Piceetum excelsae } \\
\text { Březina et Hadač } \\
\text { in Hadač et al. } 1969\end{array}$ & All relevés & $\begin{array}{l}\text { The original relevés represent } \\
\text { two different communities } \\
\text { (calcicolous: } 119,185,254 \text { vs. } \\
\text { silicicolous: } 225,253 \text { ). }\end{array}$ \\
\hline $\begin{array}{l}\text { Hadač et al. } \\
\text { (1969) }\end{array}$ & Belianske Tatry & $\begin{array}{c}\text { Chrysanthemo-Piceetum Krajina } \\
1933\end{array}$ & Pro parte & $\begin{array}{l}\text { The association name was used } \\
\text { as a pseudonym: Chrysanthemo- } \\
\text {-Piceetum sensu Hadač et al. } \\
1969 \text { non Krajina } 1933 \\
\text { (cf. Kučera in red.). }\end{array}$ \\
\hline
\end{tabular}

\begin{tabular}{|c|c|c|c|c|}
\hline $\begin{array}{l}\text { Hadač et al. } \\
\text { (1969) }\end{array}$ & Belianske Tatry & $\begin{array}{c}\text { Adenostylo-Piceetum excelsae } \\
\text { Březina et Hadač } \\
\text { in Hadač et al. } 1969\end{array}$ & Pro parte min. & \\
\hline Zlatník (1970) & Vysoké Tatry & $\begin{array}{l}\text { groups of forest types: Aceri- } \\
\text { Abieteta piceae, Abieti-piceeta }\end{array}$ & All relevés & \\
\hline $\begin{array}{l}\text { Šmarda et al. } \\
(1971)\end{array}$ & Belianske Tatry & $\begin{array}{c}\text { Sorbeto-Piceetum calcicolum } \\
\text { Pawłowski } 1956\end{array}$ & Pro parte & $\begin{array}{l}\text { Nomen fictum, thus the } \\
\text { adequate form is Sorbo-Piceetum } \\
\text { calcicolum Šmarda et al. } 1971 \text {. }\end{array}$ \\
\hline $\begin{array}{l}\text { Šmarda et al. } \\
(1971)\end{array}$ & Belianske Tatry & $\begin{array}{l}\text { Abieto-Piceetum Szafer et } \\
\text { Sokołowski } 1927\end{array}$ & All relevés & $\begin{array}{l}\text { Originally as a facies, therefore } \\
\text { the association should be cited } \\
\text { as "Abieto-Piceetum } \\
\text { Šmarda et al. 1971". }\end{array}$ \\
\hline $\begin{array}{l}\text { Šmarda et al. } \\
(1971)\end{array}$ & Belianske Tatry & Acereto-Piceetum Šmarda 1958 & All relevés & $\begin{array}{l}\text { I.e. Acero pseudoplatani-Piceetum } \\
\text { Šmarda ex Šmarda et al. } 1971\end{array}$ \\
\hline $\begin{array}{l}\text { Kubíček \& } \\
\text { Jurko (1975) }\end{array}$ & Západné Tatry & $\begin{array}{c}\text { Adenostylo-Piceetum typicum } \\
\text { Šoltés } 1971 \text { [originally nom. ined.] }\end{array}$ & Pro parte & \\
\hline Šoltés (1976) & $\begin{array}{l}\text { (higher number } \\
\text { of units) }\end{array}$ & $\begin{array}{l}\text { Adenostylo-Piceetum } \\
\text { cortusietosum }\end{array}$ & Pro parte maj. & $\begin{array}{c}\text { Only newly published relevés } \\
\text { are considered (Šoltés 1976, } \\
\text { tab. 3). }\end{array}$ \\
\hline Šoltés (1976) & Belianske Tatry & $\begin{array}{l}\text { Vaccinio myrtilli-Piceetum } \\
\text { calamagrostietosum variae }\end{array}$ & Pro parte & $\begin{array}{c}\text { Only newly published relevés } \\
\text { are considered (Šoltés } 1976, \\
\text { tab. 4). }\end{array}$ \\
\hline $\begin{array}{l}\text { Fajmonová } \\
(1978)\end{array}$ & (not specified) & $\begin{array}{c}\text { Oxalido-Piceetum } \\
\text { (Krajina 1933) Březina et Hadač } \\
\text { in Hadač et al. } 1969\end{array}$ & Pro parte & $\begin{array}{c}\text { Correct name: Oxalido-Piceetum } \\
\text { Březina et Hadač in Hadač et al. } \\
1969 \text { (cf. Kučera in red.). }\end{array}$ \\
\hline $\begin{array}{l}\text { Fajmonová } \\
(1978)\end{array}$ & (not specified) & $\begin{array}{c}\text { Cortuso-Piceetum } \\
\text { (Sillinger 1933) Šoltés } 1976\end{array}$ & Pro parte & $\begin{array}{l}\text { Nomen fictum, the most } \\
\text { probable correct form is } \\
\text { "Cortuso-Piceetum (Šoltés 1976) } \\
\text { Fajmonová 1978", } \\
\text { see explanation in the text. }\end{array}$ \\
\hline $\begin{array}{l}\text { Fajmonová } \\
(1983)\end{array}$ & $\begin{array}{c}\text { (higher number of } \\
\text { units) }\end{array}$ & $\begin{array}{c}\text { Cortuso-Piceetum } \\
\text { (Sillinger 1933) Šoltés } 1976\end{array}$ & Pro parte maj. & \\
\hline $\begin{array}{l}\text { Unar et al. } \\
(1984)\end{array}$ & Západné Tatry & $\begin{array}{c}\text { Adenostylo-Piceetum excelsae } \\
\text { Březina et Hadač in Hadač et al. } \\
1969\end{array}$ & All relevés & \\
\hline $\begin{array}{l}\text { Fajmonová } \\
(1986)\end{array}$ & $\begin{array}{c}\text { (higher number of } \\
\text { units) }\end{array}$ & $\begin{array}{c}\text { Cortuso-Piceetum (Šoltés 1976) } \\
\text { Fajmonová } 1978\end{array}$ & Pro parte & \\
\hline
\end{tabular}




\begin{tabular}{|c|c|c|c|c|}
\hline Authors & $\begin{array}{l}\text { Geomorphological } \\
\text { unit }\end{array}$ & Original name of unit & $\begin{array}{l}\text { Proportion of Carpino- } \\
\text { Fagetea relevés }\end{array}$ & Remarks \\
\hline $\begin{array}{l}\text { Uhlí̌́ová \& } \\
\text { Bernátová } \\
\text { (1986) }\end{array}$ & Vel'ká Fatra & $\begin{array}{c}\text { Phytocoenosis with Listera cordata, } \\
\text { rel. } 1 .\end{array}$ & - & $\begin{array}{l}\text { Probably more suitable } \\
\text { to classify as a marginal } \\
\text { community of the class } \\
\text { Erico-Pinetea Horvat } 1959 .\end{array}$ \\
\hline $\begin{array}{l}\text { Voško et al. } \\
(1990)\end{array}$ & ? Belianske Tatry & $\begin{array}{l}\text { Group of forest types } \\
\text { Sorbi ariae-Piceeta }\end{array}$ & All relevés & \\
\hline $\begin{array}{l}\text { Kubíček et al. } \\
(1992)\end{array}$ & Belianske Tatry & $\begin{array}{c}\text { Adenostylo-Piceetum } \\
\text { (Sillinger 1933) Šoltés } 1976\end{array}$ & Rel. 5. & \\
\hline $\begin{array}{l}\text { Kubíček et al. } \\
(1992)\end{array}$ & Belianske Tatry & $\begin{array}{c}\text { Cortuso-Piceetum (Šoltés 1976) } \\
\text { Fajmonová } 1978\end{array}$ & Rel. 7. & \\
\hline $\begin{array}{l}\text { Černušáková } \\
\text { (1994) }\end{array}$ & Západné Tatry & $\begin{array}{l}\text { Calamagrostio villosae-Piceetum } \\
\text { (Tx. 1937) Hartmann } 1953\end{array}$ & Pro parte & \\
\hline $\begin{array}{l}\text { Černušáková } \\
\text { (1994) }\end{array}$ & Západné Tatry & $\begin{array}{c}\text { Athyrio alpestre-Piceetum } \\
\text { (Hartmann 1953) Hartmann } \\
\text { et Jahn 1967) }\end{array}$ & Pro parte & \\
\hline Školek (1995a) & Ďumbierske Tatry & Higher number of units & ? All relevés & Only synoptic table. \\
\hline Školek (1995b) & Ďumbierske Tatry & Carici albae-Piceetum Školek 1995 & All relevés & \\
\hline Školek (2003) & Ďumbierske Tatry & Higher number of units & Pro parte & (Without published relevés.) \\
\hline Kanka (2008) & Belianske Tatry & Higher number of units & Pro parte & $\begin{array}{l}\text { Including Athyrio alpestris- } \\
\text {-Piceetum (rels. 3, 4) and } \\
\text { Dryopterido-Piceetum } \\
\text { (rels. } 1-4,6) \\
\end{array}$ \\
\hline Krajčí (2008) & Král'ovohol'ské Tatry & $\begin{array}{c}\text { Cortuso-Piceetum } \\
\text { (Soltés 1976) Fajmonová } 1978\end{array}$ & All relevés & $\begin{array}{l}\text { Cf. also Krajčí \& Barančok } \\
\text { (2009). }\end{array}$ \\
\hline $\begin{array}{l}\text { Valachovič et al. } \\
\text { (2019) }\end{array}$ & & Piceion excelsae & Pro parte & $\begin{array}{l}\text { Authors evaluated only } \\
\text { relevés with presence of genus } \\
\text { Soldanella. }\end{array}$ \\
\hline
\end{tabular}

Part 2. Selected examples from the mountain ranges of the Western Carpathians in Poland. The respective habitats do not occurr in Moravia and Silesia.

\begin{tabular}{|c|c|c|c|c|}
\hline Authors & $\begin{array}{l}\text { Geomorphological } \\
\text { unit }\end{array}$ & Original name of unit & $\begin{array}{l}\text { Proportion of Carpino- } \\
\text { Fagetea relevés }\end{array}$ & Remarks \\
\hline Szafer et al. (1923) & Západné Tatry & Piceetum normale Beger 1922 & Pro parte maj. & \\
\hline Szafer et al. (1923) & Západné Tatry & Piceo-Abietetum albae & All relevés & \\
\hline Szafer et al. (1927) & Západné Tatry & Piceetum excelsae normale, typical facies & Pro parte maj. & \\
\hline Szafer et al. (1927) & Západné Tatry & Piceetum excelsae normale, Polytrichum facies & Pro parte maj. & \\
\hline Szafer et al. (1927) & Západné Tatry & Abieteto-Piceetum & All relevés & \\
\hline Kulczyński (1928) & Pieniny & Piceetum excelsae & A species list & Cf. Kučera (2015b) \\
\hline Adamczyk (1962) & Západné Tatry & Piceetum excelsae tatricum & Pro parte maj. & \\
\hline $\begin{array}{l}\text { J. Matuszkiewicz } \\
\text { (1977) }\end{array}$ & - & $\begin{array}{c}\text { Galio rotundifolii-Piceetum carpaticum } \\
\text { J. Matuszkiewicz } 1977\end{array}$ & All relevés & \\
\hline $\begin{array}{l}\text { J. Matuszkiewicz } \\
(1977)\end{array}$ & (Západné Tatry) & $\begin{array}{c}\text { Polysticho-Piceetum W. Matuszkiewicz ex } \\
\text { J. Matuszkiewicz } 1977\end{array}$ & Pro parte & \\
\hline
\end{tabular}




\section{General upper line of Norway spruce altitudinal vegetation zone in the Western Carpathians}

As written above, the general upper limit of the Norway spruce altitudinal vegetation zone for the Western Carpathians was estimated in various altitudes from the elevational range 1300 to $1550 \mathrm{~m}$ a.s.l. As the uppermost yet anthropogenically lowered upper limits of mixed montane Fagus woodlands was recorded above 1360-1390 m a.s.l. (Kučera, 2012a) and natural Norway spruce zone is developed in every individual mountain range with sufficient altitudinal elevations, these traditionally accepted estimates should be reassessed as well.

According to the total altitudinal height of a particular mountain range, three categories could be differentiated when considering the Norway spruce altitudinal vegetation zone of the Western Carpathians in Slovakia:

(1) mountain ranges which do not exceed the alpine forest line (see the notes below), for example the Veporské vrchy Mts (Fabova hol'a-group), the Stolické vrchy Mts (Stolica-Kohút group);

(2) mountain ranges with the most highest isolated summits or more continuous areas exceeding this line, for example the Oravské Beskydy Mts or the Low Tatras (Nízke Tatry Mts);

(3) high mountain ranges which provided a postglacial (especially post-Boreal and post-Atlantic) refugium (cf. Krippel, 1986) for survival of Pinus cembra populations and therefore the alpine forest line (and consequently the alpine treeline) is determined by Pinus cembra (together with Larix decidua and partially Picea abies), i.e. the Tatra Mountains (equivalently to the Alps), whereby this species change has also considerable effect on the altitudinal rise of the upper forest line in comparison to the other Western Carpathian mountain ranges (Plesník, 1971); however, historical deforestation considerably reduced or destroyed native mixed Arolla pine forests, especially in their western (Západné Tatry Mts) or eastern part (Belianske Tatry Mts) (cf. Kučera (2019b, chapter 3.3), and Zięba et al. (2019), detailed distribution map in their el. appendix). ${ }^{6}$

6 As the last glacial mixed Pinus cembra woodland was present in the Podtatranská kotlina Basin lying between the Tatra Mountains and the Low Tatras as well as in other rather unexpected regions (Jankovská, 1984; Jankovská, 1991; Jankovská et al., 2002; Jankovská et al., 2018; Pokorný et al., 2015), it may be expected that Pinus cembra was a native tree of the lowest parts of Low Tatras' slopes during the more favourable periods of the Würm glaciation and after its ending the species probably survived the Atlantic period - similarly like within the Tatra Mountains - also in the uppermost elevations of the highest parts of the Low Tatras and on other ecologically extreme habitats, as for example rugged rocky terrain of some carbonate summits (Krakova hol'a Mt. etc.). Due to considerably small area they could be easily completely destroyed by later pre-/historical land
When considering the third category, it should be noted that the natural development of Picea abies (or PiceaLarix) woodlands was altitudinally considerably narrowed due to competition with mixed Arolla pine woodlands, syntaxonomically classified within either the alliance Calamagrostio variae-Pinion cembrae or the alliance Homogyno alpinae-Pinion cembrae (see Kučera, 2017). While in the lower altitudes (for example above $1400 \mathrm{~m}$ a.s.l.) spontaneously rejuvenated Pinus cembra individuals are - in ecologically favourable habitats - overshadowed by much taller Picea abies trees and they wither away, the interspecific competition has a different outcome in the higher elevations (for example above ca. $1500-1550 \mathrm{~m}$ a.s.l.) where the total height of Picea abies trees is lower: Norway spruce woodland is thus replaced with mixed Norway spruce-Arolla pine and subsequently with mixed Arolla pine woodlands. However, historical land management considerably changed the original distribution patterns of the respective woodland communities.

Historical deforestation also played an essential role for the development of the current upper forest line in the Western Carpathian mountain ranges of the second above-differentiated category. By no means could it be identified with the alpine forest line of the potential natural vegetation formed by Norway spruce woodland (cf. Plesník, 1954; Plesník, 1966; Plesník, 1975; Plesník, 1978). According to my current field knowledge, the natural upper Norway spruce forest line was generally developed above $1650 \mathrm{~m}$ a.s.l. (for the period up to 1950 1980). Only severe ecological conditions controlling the vegetation development on top regions of some elevated isolated mountains could induce occurrence of lower lying upper forest line: patches of Picea krummholz stands or, alternatively, mixed Pinus mugo scrub were originally developed before the historical deforestation.

Substantial ecological changes on largely and deeply deforested mountain slopes and ridges (as for example in the southern part of the Vel'ká Fatra Mts) significantly retard the regeneration, i.e. the reverse spontaneous secondary succession of Norway spruce forests to their former prehistorical habitats; the similar effect have Pinus mugo afforestantions in lower altitudes of the former high mountain pastures (cf. example given by Kučera (2019b)).

\section{A note to terminology of the Picea abies altitudinal vegetation zone}

Woodlands of the European Picea abies altitudinal vegetation zone are labelled with different adjectives in Central

management (for example the upper parts of the Krakova hol'a Mt. were deforested). Up to the $20^{\text {th }}$ century, segments of the natural alpine forest line did not preserve within the entire region of the Low Tatras. 
European literature, which are, likewise, applied in alternative short names of the respective altitudinal zones. The variety of terms was indicated for example by Jahn (1977, p. 478-480), see the following list:

- "high montane" in the sense of German "hochmontan", i.e. lying above the uppermost part of (further divided) montane zone (Hartmann \& Jahn, 1967; W. Matuszkiewicz, 1984);

- "upper montane" in the terms of other Polish authors who divide the montane zone to (1) the mostly $F a$ gus sylvatica-dominated "regiel dolny" and (2) the Picea abies-dominated "regiel górny" (Pawłowski, 1928; Pawłowski, 1956; Medwecka-Kornaś, 1972; W. Matuszkiewicz 1981; W. Matuszkiewicz, 2014; Mirek \& Piękoś-Mirkowa, 1992)

- "supramontane" (Domin, 1923;* Holub \& Jirásek, 1967; Neuhäuslová-Novotná, 1994; Neuhäuslová et al., 2001; Jirásek, 2002; Chytrý et al., 2013b); ${ }^{*}$ Domin applied the term also for high altitude Fagus sylvatica woodlands reaching the upper forest line (cf. also Klika, 1936), however, in the Western Carpathians such upper forest line woodlands are the result of past deep deforestation of slopes (see above);

- "oreal" (Rothmaler 1950, ca. also Haeupler, 1970; cf. Holub \& Jirásek, 1967);

- "subalpine" for the highest Norway spruce zone in old Czechoslovak botanical literature: Sillinger (1933); Klika (1936); Svoboda (1939);

- ocassionally also "altomontane" - as a replacement for German "hochmontan" (Meusel et al., 1965, p. 21).

Special case is the simultaneous application of two terms for natural Norway spruce woodlands:

- "montane" and "supramontane" (Neuhäuslová et al., 2001), "supramontane" to "subalpine" (Jirásek, 2002, p. 78);

- "high montane" of so-called mittelgebirge mountain ranges and "subalpine" for the hochgebirge mountain ranges, e.g. the Alps (Oberdorfer, 1957; Hartmann \& Jahn, 1967; Jahn, 1977; Pott, 1992; Seibert, 1992; cf. Kuoch, 1954; Exner, 2007);

- "montane" and "subalpine"/"low subalpine" or in German also "tiefsubalpin" to two groups of altitudinally differentiated coniferous, especially Norway spruce communities of the Alps (Ellenberg, 1963 and later editions; Mayer \& Hofmann, 1969; Elenberg \& Klötzli, 1972; Zukrigl, 1973; Mayer, 1974; Mayer, 1984; Horvat et al., 1974; Pignatti, 1998; cf. Braun-Blanquet et al., 1939; Braun-Blanquet et al., 1954; Kuoch, 1954; Pott, 1992; Exner, 2007; Chifu, 2014; Pignatti \& Pignatti, 2014); see also similar approach of Sillinger (1933) and Svoboda (1939) for Tatra Mountains' and Low Tatras' Norway spruce forests;
- "upper montane" and "subalpine" (Coldea, 2015);

- "altimontane" and "subalpine" (Šilc \& Čarni, 2012; Juvan et al., 2013);

- "oreal" and "subalpine" (Haeupler, 1970; cf. Jahn, 1977).

Terminological unification seems to be difficult because the particular terms are associated with different traditional use within the individual European regions. Instead of lengthy descriptions and characterizations of individual approaches and subsequent additional reasoning, the following short proposal should be presented in this place.

The term alpine forest line (closely connected with the alpine tree line) (see Körner, 2012) should be chosen as the crucial terminological base: the alpine forest line represents one of the several types of general climate-driven forest lines (arctic forest line etc.); other special forest lines - e.g. orographic, edaphic - are natural ecological limits of different kind as they are "disrupting" the general patterns of vegetation development of a particular region/area (cf. Wraber, 1970; Plesník, 1971; Mayer, 1984; Ellenberg, 1996). In contrast to the evaluation of Jeník \& Lokvenc (1962), the mechanically changed course of upper forest line (avalanches, snow patches [nivation], debris flows etc.) could not be labelled as part of an "alpine forest line" because the absence of tree stands is there not caused by the alpine climate. Use of the term "artificial alpine forest line" (Jeník \& Lokvenc, 1962, p. 24) is controversial as well, especially if the respective anthropogenic modification resulted in decrease of the upper forest line in tens or hundreds of metres (cf. Plesník, 1971; Plesník, 1978; Wraber, 1970).

The alpine climate inhibits formation of woodland communities and only non-forest plant communities are present, with the special case of krummholz belt in lower elevations of the alpine region of some mountain ranges (Domin, 1923; Rothmaler, 1955; Meusel et al., 1965, p. 21; Holub \& Jirásek, 1967). A krummholz belt formed by Pinus mugo [s. str.] scrub is a distinctive feature of the Carpathian mountain ranges and other European mountain ranges as Sudetes (the Krkonoše Mts.) (Holub \& Jirásek, 1967; Medwecka-Kornaś, 1972), Dinarides, Apennines and also of the great part of the Alps (Jalas \& Suominen, 1973, map 169; Horvat et al., 1974; Ellenberg, 1996, fig. 226; Pignatti et al., 2017); however, it could be formed by Picea abies-krummholz (primarily in the Hrubý Jeseník Mts.) or other (also broadleaf) species in the mountain ranges where Pinus mugo (or Picea abies) does not naturally forms an altitudinal vegetation zone.

Therefore the adjective term "alpine" (and its derivatives, e.g. subalpine) should be only associated with vegetation types above the alpine forest line (see already Meusel et al., 1965), while the various mountain forest 
types below this line - and reaching this line - should be labelled with the adjective term "montane" (and its various derivatives) (see Domin, 1923; Holub \& Jirásek, 1967; Mirek \& Piękoś-Mirkowa, 1992) as indicated in the following scheme: ${ }^{7}$

\begin{tabular}{|c|c|c|}
\hline \multirow{2}{*}{ nival (sensu lato) } & $\begin{array}{c}\text { nival } \\
\text { subnival }\end{array}$ & nival sensu stricto \\
\hline $\begin{array}{c}\text { climatic perennial } \\
\text { snow line }\end{array}$ & $\begin{array}{c}\text { climatic perennial } \\
\text { snow line }\end{array}$ & $\begin{array}{c}\text { climatic perennial } \\
\text { snow line }\end{array}$ \\
\hline \multirow{2}{*}{ alpine (sensu lato) } & alpine (upper, lower)* & alpine sensu stricto \\
\cline { 2 - 3 } & subalpine & \\
\hline alpine forest line & alpine forest line & alpine forest line \\
\hline \multirow{2}{*}{$\begin{array}{c}\text { montane } \\
\text { (sensu lato) }\end{array}$} & supramontane** & \\
\cline { 2 - 3 } & upper montane & montane sensu \\
lower montane & \\
\cline { 2 - 3 } $\begin{array}{c}\text { complex of planar } \\
\text { and colline zones }\end{array}$ & submontane & \\
\hline
\end{tabular}

* An analogous term "supraalpin" (to supramontane) is applicable for special non-European climate-driven high-altitude regions extensively without continuous snow and simultaneously vegetation cover (for example region of Ojos del Salado in the Andes, Gspurning et al. (2006)). The "vegetation-free" rocky steep slopes/walls of peaks of the High Tatras (or in the montane-alpine elevations the Alps) does not belong here, because they are caused by orographic, not climatic conditions.

** In some European mountain ranges, Picea abies does not form an altitudinal zone due to phytochorological or other reasons (the Massif Central, the Vosges, as well as some Southeastern European mountain ranges; Issler, 1942; Horvat et al., 1974; Boeuf et al., 2014), therefore the physiognomically distict (coniferous) uppermost subzone of the montane zone (sensu lato) is not developed. Their uppermost Fagus sylvatica horizon corresponds to other beech woodlands of higher montane elevations (e.g. upper montane Western Carpathian mixed Fagus woodland above ca. 1250-1300 m a.s.l.) and, consequently, as such it should not be labelled as a supramontane zone. However, physiognomically distinct supramontane zone could be formed by other species when Picea abies is naturally absent - see the example of woodlands of Pinus uncinata Ramond ex DC s. str. in the Pyrenees (Vigo, 1979).

The Tatra Mountains (and few other European regions, Jalas \& Suominen, 1973) with their hochgebirge character are distinct by the native occurrence of Pinus cembra preserved in the form of postglacial refugia (Jankovská, 1991). As the presence of this tree species considerably transforms the altitudinal pattern

7 Position of "sub-zones" are following the classification of Rothmaler (1955) and Löve (1970, section Etymology); however, the label "subalpine zone" is here applied for the krummholz zone only (see above). of the alpine forest line in comparison to other (mittelgebirge) mountain ranges with native Norway spruce belt and simultaneously with Arolla pine absence (Plesník, 1971; Kučera, 2012a; Zięba et al., 2019), the respective high mountain mixed P. cembra woodlands could be labelled with the adjective "altimontane" (= occurring in the very high montane altitudes). ${ }^{8}$ In the Pyrenees the similar role is taken by high-altitude Pinus uncinata woodland (cf. Rivas-Martínez, 1968).

*** See Haeupler (1970).

According to this proposal, natural Picea abies altitudinal vegetation zone (including the mixed Picea-Larix decidua communities of some regions) in the mountain ranges of Central Europe (with Alps) to Southeastern Europe (Southern Carpathians) would be labelled as "supramontane zone", irrespective of the total altitudinal limits reached in the particular region. Only those Alpic Norway spruce forests of the so-called Zentralalpen zone, which altitudinally correspond to the Randalpen upper montane mixed Fagus forests, should be labelled as woodlands of the montane zone s. l.; however, their spatial distribution should be revised (see below p. 140).

The term "oreal" (proposed by Rothmaler (1950)) is based on the Greek equivalent to Latin expressions "montana, montanus"; it was also preceded by the older Domin's term "supramontane" (Domin, 1923) which is therefore here prioritised.

However, the term "oreal" could be advantageously applied for plant species which have their altitudinal centre of distribution in higher montane altitudes as well as in subalpine altitudinal zone, for example Adenostyles alliariae, Athyrium alpestre, Calamagrostis villosa, Cicerbita alpina, Doronicum austriacum, Homogyne alpina, Gentiana asclepiadea, Luzula sylvatica, (Streptopus amplexifolius, Trientalis europaea) etc. ${ }^{9}$ These are species which grow (for example in the Western Carpathians) mainly in the uppermost horizon of montane mixed Fagus sylvatica woodlands, in the supramontane Picea abies forests, in the subalpine Pinus mugo krummholz, and at the same time in montane to subalpine agradal ${ }^{10}$ tall-forb communities

8 Juvan et al. (2013) used the labels "altimontane" and "subalpine" for two groups of coniferous forests which could correspond to the above proposed term "supramontane". However, the very high frequencies of Fagus sylvatica (73\% and 58\%) indicate the considerable proportion of data recorded in changed secondary forests, thus belonging to the class Carpino-Fagetea (see above p. 111-113).

9 The respective species are incorrectly labelled as "subalpine species" in Slovak forest science handbooks (e.g. Randuška (1986) and later editions) despite the fact that their altitudinal centre of distribution is below the (potential natural) alpine forest line which lies above $1650 \mathrm{~m}$ a.s.l., respectively above 1800 a.s.l. for Pinus cembra forests of the Tatra Mountains.

10 See above for the differentiation between Latin "zona" and "gradus"; thus the terms "azonal/extrazonal" and "agradal/extragradal" have separate distinct meanings. 
of the classes Mulgedio-Aconitetea Hadač et Klika in Klika 1944 and Betulo carpaticae-Alnetea Rejmánek ex Boeuf et al. in Boeuf et al. 2014.

\section{Associations of calcareous Norway spruce woodlands in Slovakia}

Six basic natural calcicolous Norway spruce plant communities are differentiated in this syntaxonomical revision. They are here arranged according to ecological gradient provided by carbonate rocks.

Stands of the association Seslerio caeruleae-Piceetum are developed on edaphically extreme carbonate habitats with shallow soils. The three subcommunities of the association
Cirsio erisithalis-Piceetum correspond to the continual improvement of habitat conditions (higher soil depth and humidity). On even deeper soils are developed stands of the association Fragario vescae-Piceetum or, alternatively, more widespread tall-forb woodlands of the association Adenostylo alliariae-Piceetum which are bound to habitats with favourable water regime. The last two associations represent marginal units of calcicolous natural Norway spruce woodlands because the presence of calciphytes is reduced. The association Mnio spinosi-Piceetum is mostly negatively differentiated; however, it is related with phytocoenoses of the previous association. The stands of $\mathrm{Hi}$ eracio murorum-Piceetum are developed over mostly deep soils acidified in their uppermost horizon.

Table 2. Statistical comparison of associations of the alliance Cortuso matthioli-Piceion abietis P. Kučera 2022 in Slovakia with values of constancy $(\%)$ and fidelity $(\varphi(\times 100) \geq 25)$ in the exponent.

Table 2. Statistična primerjava asociacij zveze Cortuso matthioli-Piceion abietis P. Kučera 2022 na Slovaškem s prikazano stalnostjo $(\%)$ in nadpisano navezanostjo $(\varphi(\times 100) \geq 25)$.

Field and ground layer species represented in only one column are omitted.

Group 1 - Seslerio caeruleae-Piceetum abietis Fajmonová 1978 nom. corr. et nom. cons. propos.

Group 2 - Cirsio erisithalis-Piceetum abietis Fajmonová et P. Kučera in P. Kučera 2022 ass. nov.

Group 3 - Fragario vescae-Piceetum abietis P. Kučera ass. nov. prov.

Group 4 - Adenostylo alliariae-Piceetum abietis Samek et al. 1957 nom. corr. et nom. cons. propos.

Group 5 - Mnio spinosi-Piceetum abietis Hadač et al. 1969 nom. corr.

Group 6 - Hieracio murorum-Piceetum abietis P. Kučera 2022 ass. nov.

The quantity of asterisks in the second column express the highest state of conventional levels of the statistical significance $(0.05$, 0.01 and 0.001 , Fisher's exact test) for the particular species. In the case of differential species for multiple units such indication could have alternative states.

\begin{tabular}{|c|c|c|c|c|c|c|c|}
\hline Group No. & & 1 & 2 & 3 & 4 & 5 & 6 \\
\hline No. of relevés & & 14 & 13 & 6 & 28 & 13 & 6 \\
\hline Average number of species & & 54 & 53 & 49 & 47 & 23 & 31 \\
\hline \multicolumn{8}{|l|}{ Tree and shrub species } \\
\hline \multicolumn{8}{|l|}{ Canopy $\left(\mathrm{E}_{\mathbf{3}}\right)$} \\
\hline Picea abies & & $100^{-}$ & $100^{-}$ & $100^{-}$ & $96^{-}$ & $100^{-}$ & $100^{-}$ \\
\hline Sorbus aucuparia & *** & $21^{-}$ & $85^{55.0}$ & $17^{-}$ & $43^{-}$ & $8^{-}$ &.$^{-}$ \\
\hline Acer pseudoplatanus & * & $7^{-}$ & $38^{25.3}$ & $\mathbf{5 0}^{39.0}$ & $7^{-}$ &.$^{-}$ &.$^{-}$ \\
\hline Larix decidua & &.$^{-}$ &.$^{-}$ & $17^{-}$ & $7^{-}$ & $15^{-}$ &.$^{-}$ \\
\hline Sorbus aria agg. & *** &.$^{-}$ & 3152.0 &.$^{-}$ &.$^{-}$ & $\cdot^{-}$ &.$^{-}$ \\
\hline Abies alba & &.$^{-}$ &.$^{-}$ & $17^{-}$ &.$^{-}$ & $8^{-}$ &.$^{-}$ \\
\hline Fagus sylvatica & &.$^{-}$ & $8^{-}$ &.$^{-}$ &.$^{-}$ &.$^{-}$ &.$^{-}$ \\
\hline Salix silesiaca & &.$^{-}$ &.$^{-}$ &.$^{-}$ & $4^{-}$ &.$^{-}$ &.$^{-}$ \\
\hline \multicolumn{8}{|l|}{ Understorey $\left(\mathrm{E}_{2}\right)$} \\
\hline Sorbus aucuparia & * & $7^{-}$ & $38^{-}$ & $17^{-}$ & $46^{26.4}$ & $23^{-}$ &.$^{-}$ \\
\hline Picea abies & & $21^{-}$ & $23^{-}$ & $33^{-}$ & $18^{-}$ & $23^{-}$ &.$^{-}$ \\
\hline Ribes petraeum & ** &.$^{-}$ & $8^{-}$ & $17^{-}$ & $32^{27.8}$ & $15^{-}$ &.$^{-}$ \\
\hline Pinus mugo & ** & $43^{40.1}$ & $31^{-}$ & $\cdot^{-}$ & $4^{-}$ &.$^{-}$ &.$^{-}$ \\
\hline Salix silesiaca & * & $7^{-}$ & $31^{35.0}$ &.$^{-}$ & $14^{-}$ &.$^{-}$ &.$^{-}$ \\
\hline Acer pseudoplatanus & * &.$^{-}$ & $31^{27.6}$ & $33^{-}$ & $4^{-}$ &.$^{-}$ & $\cdot^{-}$ \\
\hline Fagus sylvatica & &.$^{-}$ & $15^{-}$ & $\cdot^{-}$ & $4^{-}$ &.$^{-}$ &.$^{-}$ \\
\hline
\end{tabular}




\begin{tabular}{|c|c|c|c|c|c|c|c|}
\hline Group No. & & 1 & 2 & 3 & 4 & 5 & 6 \\
\hline Sorbus aria agg. & ** &.$^{-}$ & $23^{44.7}$ &.$^{-}$ &.$^{-}$ &.$^{-}$ &.$^{-}$ \\
\hline Lonicera nigra & &.$^{-}$ &.$^{-}$ & $17^{-}$ & $7^{-}$ &.$^{-}$ &.$^{-}$ \\
\hline Rosa pendulina & &.$^{-}$ &.$^{-}$ & $17^{-}$ & $4^{-}$ &.$^{-}$ &.$^{-}$ \\
\hline Daphne mezereum & & $\cdot^{-}$ & $8^{-}$ & $\cdot^{-}$ &.$^{-}$ &.$^{-}$ & $\cdot^{-}$ \\
\hline Salix caprea & & $\cdot^{-}$ &.$^{-}$ & $\cdot^{-}$ & $4^{-}$ &.$^{-}$ & $\cdot^{-}$ \\
\hline Betula carpatica & & $\cdot^{-}$ & $\cdot^{-}$ & $\cdot^{-}$ & $4^{-}$ &.$^{-}$ & $\cdot^{-}$ \\
\hline Ribes alpinum & &.$^{-}$ &.$^{-}$ &.$^{-}$ & $4^{-}$ &.$^{-}$ &.$^{-}$ \\
\hline Sambucus racemosa & &.$^{-}$ &.$^{-}$ &.$^{-}$ & $4^{-}$ &.$^{-}$ &.$^{-}$ \\
\hline Ribes uva-crispa & &.$^{-}$ &.$^{-}$ &.$^{-}$ &.$^{-}$ & $8^{-}$ &.$^{-}$ \\
\hline \multicolumn{8}{|l|}{$\mathbf{E}_{\mathbf{1}}$} \\
\hline Sorbus aucuparia & * & $93^{27.5}$ &.$^{-}$ & $67^{-}$ & $75^{-}$ & $62^{-}$ & $83^{-}$ \\
\hline Daphne mezereum & *** & $93^{38.3}$ & $85^{30.9}$ & $83^{-}$ & $39^{-}$ &.$^{-}$ &.$^{-}$ \\
\hline Lonicera nigra & ** & $64^{33.9}$ & $23^{-}$ & $33^{-}$ & $57^{26.9}$ &.$^{-}$ &.$^{-}$ \\
\hline Picea abies & * &.$^{-}$ & $31^{-}$ & $50^{-}$ & $36^{-}$ & $31^{-}$ & $\mathbf{8 3}^{41.3}$ \\
\hline Ribes petraeum & *** &.$^{-}$ &.$^{-}$ & $50^{-}$ & $61^{41.3}$ & $23^{-}$ &.$^{-}$ \\
\hline Acer pseudoplatanus & * & $14^{-}$ & $23^{-}$ & $17^{-}$ & $25^{-}$ & $\cdot^{-}$ & $\mathbf{6} 7^{44.2}$ \\
\hline Salix silesiaca & *** & $7^{-}$ & $46^{46.2}$ & $\cdot^{-}$ & $4^{-}$ &.$^{-}$ & $17^{-}$ \\
\hline Rosa pendulina & & $21^{-}$ & $15^{-}$ & $17^{-}$ & $7^{-}$ &.$^{-}$ &.$^{-}$ \\
\hline Fagus sylvatica & *** &.$^{-}$ &.$^{-}$ &.$^{-}$ & $4^{-}$ &.$^{-}$ & $50^{64.4}$ \\
\hline Ribes uva-crispa & * &.$^{-}$ & $\cdot^{-}$ &.$^{-}$ & $14^{34.9}$ &.$^{-}$ & $\cdot^{-}$ \\
\hline Ribes alpinum & * &.$^{-}$ &.$^{-}$ &.$^{-}$ & $11^{30.2}$ &.$^{-}$ &.$^{-}$ \\
\hline Sorbus aria agg. & * &.$^{-}$ & $15^{36.3}$ &.$^{-}$ &.$^{-}$ &.$^{-}$ &.$^{-}$ \\
\hline Abies alba & &.$^{-}$ & $\cdot^{-}$ & $17^{-}$ &.$^{-}$ &.$^{-}$ &.$^{-}$ \\
\hline Pinus cembra & &.$^{-}$ &.$^{-}$ &.$^{-}$ & $4^{-}$ &.$^{-}$ &.$^{-}$ \\
\hline Pinus mugo & &.$^{-}$ &.$^{-}$ &.$^{-}$ &.$^{-}$ & $8^{-}$ &.$^{-}$ \\
\hline
\end{tabular}

Diagnostic field layer species $\left(\mathrm{E}_{1}\right)$

Ranunculus breyninus

*** 8691.3

Phyteuma orbiculare

*** $\quad 79^{86.8}$

Crepis jacquinii

Vaccinium vitis-idaea

Carex ornithopoda

Carex digitata

Carex sempervirens ssp. tatrorum

Carduus glaucinus

*** $\quad 79^{84.4}$

*** $\quad 71^{82.2}$

*** $\quad 866^{75.4}$

*** $\quad 57^{72.5}$

*** $\quad 79^{69.3}$

*** $\quad 57^{61.8}$

Swertia perennis

Tofieldia calyculata

Maianthemum bifolium

Clematis alpina

Calamagrostis varia

Poa alpina

Festuca tatrae

Orthilia secunda

Campanula rotundifolia agg.

Fragaria vesca

** $\quad 6459.3$

*** $\quad 36^{56.3}$

*** $\quad 50^{56.1}$

*** $\quad 86^{54.4}$

*** $\quad 7952.3$

*** $\quad 8651.4$

*** $\quad 4350.4$

** $\quad 36^{46.4}$

** $\quad 29^{46.1}$

** $\quad 4343.7$

*** $\quad 7^{41.5}$

Galium anisophyllon

Lathyrus vernus

* $\quad 2138.6$

* $21^{34.5}$ 


\begin{tabular}{|c|c|c|c|c|c|c|c|}
\hline Group No. & & 1 & 2 & 3 & 4 & 5 & 6 \\
\hline Aquilegia vulgaris & ** & $36^{33.9}$ &.$^{-}$ & $33^{-}$ &.$^{-}$ &.$^{-}$ &.$^{-}$ \\
\hline Corallorrhiza trifida & * & $29^{31.8}$ &.$^{-}$ &.$^{-}$ &.$^{-}$ & $23^{-}$ &.$^{-}$ \\
\hline Melica nutans & ** & $29^{31.1}$ & $8^{-}$ & $17^{-}$ &.$^{-}$ & $\cdot^{-}$ &.$^{-}$ \\
\hline Polygonatum verticillatum & * & $93^{31.1}$ & $77^{-}$ & $67^{-}$ & $68^{-}$ & $31^{-}$ & $17^{-}$ \\
\hline Gymnocarpium robertianum & * & $29^{30.0}$ & $23^{-}$ &.$^{-}$ & $4^{-}$ &.$^{-}$ &.$^{-}$ \\
\hline Homogyne alpina & * & $100^{27.7}$ & $77^{-}$ & $17^{-}$ & $79^{-}$ & $62^{-}$ & $100^{-}$ \\
\hline Astrantia major & * & $71^{25.0}$ & $69^{-}$ & $67^{-}$ & $39^{-}$ & $15^{-}$ &.$^{-}$ \\
\hline Cystopteris montana & *** &.$^{-}$ & $54^{70.2}$ &.$^{-}$ &.$^{-}$ & $\cdot^{-}$ &.$^{-}$ \\
\hline Dentaria enneaphyllos & *** & $21^{-}$ & $85^{69.7}$ & $17^{-}$ & $4^{-}$ &.$^{-}$ &.$^{-}$ \\
\hline Rubus saxatilis & *** & $43^{23.6}$ & $77^{60.9}$ &.$^{-}$ &.$^{-}$ & $8^{-}$ &.$^{-}$ \\
\hline Festuca carpathica & *** &.$^{-}$ & $38^{58.5}$ &.$^{-}$ &.$^{-}$ &.$^{-}$ &.$^{-}$ \\
\hline Asplenium viride & *** & $57^{-}$ & $92^{53.4}$ & $33^{-}$ & $29^{-}$ &.$^{-}$ &.$^{-}$ \\
\hline Ranunculus nemorosus & *** &.$^{-}$ & $31^{52.0}$ &.$^{-}$ &.$^{-}$ &.$^{-}$ &.$^{-}$ \\
\hline Laserpitium latifolium & ** &.$^{-}$ & $31^{48.2}$ & $\cdot^{-}$ & $4^{-}$ &.$^{-}$ &.$^{-}$ \\
\hline Cortusa matthioli & *** & $64^{-}$ & $100^{47.1}$ & $67^{-}$ & $54^{-}$ &.$^{-}$ &.$^{-}$ \\
\hline Allium victorialis & $* * *$ &.$^{-}$ & $38^{45.3}$ &.$^{-}$ &.$^{-}$ &.$^{-}$ & $17^{-}$ \\
\hline Crepis paludosa & *** & $43^{-}$ & $85^{45.1}$ & $50^{-}$ & $39^{-}$ &.$^{-}$ &.$^{-}$ \\
\hline Trollius altissimus & ** &.$^{-}$ & $23^{44.7}$ & $\cdot^{-}$ &.$^{-}$ &.$^{-}$ &.$^{-}$ \\
\hline Mercurialis perennis & *** & $57^{19.1}$ & $85^{44.6}$ & $67^{-}$ & $4^{-}$ & $8^{-}$ &.$^{-}$ \\
\hline Viola biflora & *** & $64^{-}$ & $92^{43.5}$ & $17^{-}$ & $50^{-}$ & $8^{-}$ & $33^{-}$ \\
\hline Geranium sylvaticum & *** & $43^{-}$ & $85^{41.3}$ & $50^{-}$ & $43^{-}$ &.$^{-}$ & $17^{-}$ \\
\hline Poa stiriaca & ** & $29^{24.7}$ & $38^{38.7}$ &.$^{-}$ &.$^{-}$ &.$^{-}$ &.$^{-}$ \\
\hline Veratrum album ssp. lobelianum & ** & $36^{-}$ & $77^{38.6}$ &.$^{-}$ & $54^{-}$ & $31^{-}$ & $17^{-}$ \\
\hline Campanula serrata & *** &.$^{-}$ & $46^{36.7}$ & $17^{-}$ &.$^{-}$ &.$^{-}$ & $33^{-}$ \\
\hline Cyanus mollis & * &.$^{-}$ & $15^{36.3}$ &.$^{-}$ &.$^{-}$ &.$^{-}$ &.$^{-}$ \\
\hline Symphytum tuberosum & * & $\cdot^{-}$ & $15^{36.3}$ & $\cdot^{-}$ & $\cdot^{-}$ &.$^{-}$ & $\cdot^{-}$ \\
\hline Saxifraga rotundifolia & * & $\cdot^{-}$ & $15^{36.3}$ & $\cdot^{-}$ & $\cdot^{-}$ &.$^{-}$ &.$^{-}$ \\
\hline Primula elatior & ** & $64^{-}$ & $\mathbf{1 0 0}^{35.6}$ & $83^{-}$ & $64^{-}$ & $38^{-}$ & $17^{-}$ \\
\hline Gentiana asclepiadea & ** & $64^{-}$ & $\mathbf{9 2}^{32.8}$ & $83^{-}$ & $57^{-}$ & $38^{-}$ &.$^{-}$ \\
\hline Melampyrum sylvaticum & ** & $43^{19.4}$ & $54^{30.9}$ & $33^{-}$ &.$^{-}$ & $15^{-}$ & $\cdot-$ \\
\hline Geum rivale & * & $7^{-}$ & $38^{30.3}$ & $17^{-}$ & $25^{-}$ &.$^{-}$ &.$^{-}$ \\
\hline Phyteuma spicatum & * & $71^{-}$ & $85^{28.8}$ & $67^{-}$ & $54^{-}$ & $38^{-}$ &.$^{-}$ \\
\hline Actaea spicata & ** &.$^{-}$ &.$^{-}$ & $50^{56.8}$ & $14^{-}$ &.$^{-}$ &.$^{-}$ \\
\hline Aconitum variegatum & ** & $14^{-}$ & $8^{-}$ & $67^{54.5}$ & $25^{-}$ &.$^{-}$ &.$^{-}$ \\
\hline Digitalis grandiflora & ** &.$^{-}$ &.$^{-}$ & $33^{54.2}$ &.$^{-}$ &.$^{-}$ &.$^{-}$ \\
\hline Polypodium vulgare & ** &.$^{-}$ &.$^{-}$ & $33^{54.2}$ &.$^{-}$ &.$^{-}$ &.$^{-}$ \\
\hline Polystichum aculeatum & * &.$^{-}$ &.$^{-}$ & $33^{50.6}$ & $4^{-}$ &.$^{-}$ &.$^{-}$ \\
\hline Geranium robertianum & * &.$^{-}$ &.$^{-}$ & $33^{50.6}$ & $4^{-}$ &.$^{-}$ &.$^{-}$ \\
\hline Dryopteris filix-mas & ** & $14^{-}$ & $46^{-}$ & 10045.9 & $57^{-}$ & $8^{-}$ & $67^{-}$ \\
\hline Campanula trachelium & * &.$^{-}$ & $8^{-}$ & $33^{44.2}$ & $4^{-}$ &.$^{-}$ &.$^{-}$ \\
\hline Campanula persicifolia & * & $7^{-}$ & $8^{-}$ & $33^{41.6}$ &.$^{-}$ &.$^{-}$ &.$^{-}$ \\
\hline Delphinium elatum & * &.$^{-}$ & $15^{-}$ & $33^{39.0}$ & $4^{-}$ &.$^{-}$ &.$^{-}$ \\
\hline Doronicum austriacum & *** & $\cdot^{-}$ & $8^{-}$ & $\cdot^{-}$ & $64^{67.2}$ & $8^{-}$ &.$^{-}$ \\
\hline Leucanthemum rotundifolium & *** & $29^{-}$ & $23^{-}$ & $17^{-}$ & $82^{58.9}$ &.$^{-}$ &.$^{-}$ \\
\hline Milium effusum & *** & $7^{-}$ & $15^{-}$ &.$^{-}$ & $50^{52.0}$ &.$^{-}$ &.$^{-}$ \\
\hline Cicerbita alpina & *** & $7^{-}$ & $38^{-}$ & $33^{-}$ & $82^{49.9}$ & $23^{-}$ &.$^{-}$ \\
\hline Dentaria glandulosa & ** &.$^{-}$ &.$^{-}$ &.$^{-}$ & $21^{43.0}$ &.$^{-}$ &.$^{-}$ \\
\hline
\end{tabular}




\begin{tabular}{|c|c|c|c|c|c|c|c|}
\hline Group No. & & 1 & 2 & 3 & 4 & 5 & 6 \\
\hline Sesleria tatrae & ** &.$^{-}$ &.$^{-}$ &.$^{-}$ & $18^{39.2}$ &.$^{-}$ &.$^{-}$ \\
\hline Arabis alpina & * &.$^{-}$ &.$^{-}$ &.$^{-}$ & $14^{34.9}$ &.$^{-}$ &.$^{-}$ \\
\hline Oreogeum montanum & * &.$^{-}$ &.$^{-}$ &.$^{-}$ & $14^{34.9}$ &.$^{-}$ &.$^{-}$ \\
\hline Aconitum firmum & ** & $7^{-}$ & $15^{-}$ &.$^{-}$ & $29^{32.1}$ &.$^{-}$ &.$^{-}$ \\
\hline Epilobium montanum & *** & $14^{-}$ & $8^{-}$ & $67^{-}$ & $\mathbf{6 4}^{30.8}$ & $23^{-}$ & $17^{-}$ \\
\hline Geranium palustre & * &.$^{-}$ & $\cdot^{-}$ &.$^{-}$ & $11^{30.2}$ &.$^{-}$ &.$^{-}$ \\
\hline Adenostyles alliariae & *** & $43^{-}$ & $46^{-}$ & $67^{-}$ & $96^{28.6}$ & $62^{-}$ & $83^{-}$ \\
\hline Chrysosplenium alternifolium & ** &.$^{-}$ & $23^{-}$ & $17^{-}$ & $39^{28.5}$ &.$^{-}$ & $17^{-}$ \\
\hline Galeobdolon luteum agg. & ** & $21^{-}$ & $54^{-}$ & $50^{-}$ & $\mathbf{6 8}^{26.9}$ & $38^{-}$ &.$^{-}$ \\
\hline Veronica officinalis & * &.$^{-}$ &.$^{-}$ &.$^{-}$ &.$^{-}$ & $15^{36.3}$ &.$^{-}$ \\
\hline Soldanella carpatica & ** & $36^{-}$ & $31^{-}$ &.$^{-}$ & $18^{-}$ & $69^{33.3}$ & $50^{-}$ \\
\hline Agrostis capillaris & **** &.$^{-}$ &.$^{-}$ &. &.$^{-}$ &.$^{-}$ & $83^{89.8}$ \\
\hline Calamagrostis villosa & *** &.$^{-}$ & $31^{-}$ &.$^{-}$ & $25^{-}$ & $31^{-}$ & $100^{66.6}$ \\
\hline Luzula luzuloides & *** & $29^{-}$ &.$^{-}$ & $33^{-}$ & $25^{-}$ &.$^{-}$ & $100^{66.5}$ \\
\hline Deschampsia cespitosa & ** &.$^{-}$ &. &.$^{-}$ & $7^{-}$ & $8^{-}$ & $50^{56.5}$ \\
\hline Veronica chamaedrys & ** &.$^{-}$ &.$^{-}$ &.$^{-}$ &.$^{-}$ &.$^{-}$ & $33^{54.2}$ \\
\hline Ranunculus repens & ** &.$^{-}$ &.$^{-}$ &.$^{-}$ &.$^{-}$ &.$^{-}$ & $33^{54.2}$ \\
\hline Poa annua & ** &.$^{-}$ &.$^{-}$ &.$^{-}$ &.$^{-}$ &.$^{-}$ & $33^{54.2}$ \\
\hline Anthoxanthum odoratum & ** &.$^{-}$ &.$^{-}$ &.$^{-}$ &.$^{-}$ &.$^{-}$ & $33^{54.2}$ \\
\hline Rubus idaeus & ** & $7^{-}$ & $8^{-}$ & $67^{-}$ & $57^{11.0}$ & $31^{-}$ & $100^{49.5}$ \\
\hline Athyrium filix-femina & ** & $14^{-}$ & $15^{-}$ & $17^{-}$ & $39^{-}$ & $23^{-}$ & $\mathbf{8 3}^{49.2}$ \\
\hline Dryopteris carthusiana agg. & * & $14^{-}$ & $31^{-}$ & $33^{-}$ & $71^{20.9}$ & $38^{-}$ & $100^{46.5}$ \\
\hline
\end{tabular}

Diagnostic field layer species for two or more associations

Sesleria caerulea

Bellidiastrum michelii

Pimpinella major

Soldanella marmarossiensis agg.

Heracleum sphondylium

Cardaminopsis arenosa agg.

Cirsium erisithales

Valeriana tripteris

Mycelis muralis

Polystichum lonchitis

Pyrethrum clusii

Tithymalus amygdaloides

Chaerophyllum hirsutum

Galium schultesii

Stellaria nemorum

Calamagrostis arundinacea

Other field layer species $\left(\mathrm{E}_{1}\right)$

Oxalis acetosella

Luzula sylvatica ssp. sylvatica

Senecio nemorensis agg.

Vaccinium myrtillus

Hieracium murorum

Prenanthes purpurea

Myosotis sylvatica
**** $\quad 100 \stackrel{69.2}{ } \quad 77 \underline{46.5}$

추 $\quad 93 \frac{66.5}{62} \quad 2^{34.9}$

*** $\quad \mathbf{6 4} \underline{48.9} \quad 46^{28.8}$

**/* $\quad 79 \underline{38.4} \quad 31^{-}$

*** $\quad \mathbf{7 9}^{35.8} \quad \mathbf{1 0 0} 55.4$

*** $\quad 644^{34.1} \quad 92$ 61.6

*** $\quad \mathbf{8 6} \stackrel{33.9}{\underline{100}} \stackrel{46.7}{ }$

** $\quad 100+32.3$

** $\quad 71^{26.7}$

$100 \frac{32.3}{2}$

$15^{-}$

$* * * * * \quad 64^{26.3}$

$69 \stackrel{30.9}{3}$

$69 \underline{37.8}$

$* * * * *$

***/* .

**** 21

${ }^{* * / *} \quad 36^{-}$

$46 \frac{35.5}{5}$

$\mathbf{6 2}^{32.2}$

69

$* * * / * *$

$\stackrel{* * * / *}{*}$
$15^{-}$

$86 \stackrel{30.2}{\underline{3}}$
$23^{-}$

$83^{28.1}$

$\cdot^{-}$

. 4

$8^{-}$

33

$64^{25.2}$

$15^{-}$

$33^{-}$

25

$17^{-}$

$4^{-}$

$83^{-}$

$18^{-}$

$100^{-}$

$71^{-}$

$23^{-}$

$67^{-}$

$21^{-}$

$77^{31.7}$

$50^{-}$

$25^{-}$

$8^{-}$

$67^{35.3}$

$11^{-}$

$\mathbf{5 0}^{40.1}$

$4^{-}$

$57^{27.9}$

$17^{-}$

21

$15^{-}$

68

$8^{-}$

29 -

$8^{-}$

$83^{28.1}$

\begin{tabular}{|c|c|c|c|c|}
\hline $69^{-}$ & $83^{-}$ & $96^{14.0}$ & $85^{-}$ & $100^{-}$ \\
\hline $100^{22.7}$ & $67^{-}$ & $79^{-}$ & $46^{-}$ & $100^{-}$ \\
\hline $54^{-}$ & $100^{-}$ & $96^{19.9}$ & $85^{-}$ & $83^{-}$ \\
\hline $92^{-}$ &.$^{-}$ & $64^{-}$ & $77^{-}$ & $100^{-}$ \\
\hline $77^{-}$ & $67^{-}$ & $50^{-}$ & $38^{-}$ & $83^{-}$ \\
\hline $69^{-}$ & $67^{-}$ & $57^{-}$ & $54^{-}$ & $33^{-}$ \\
\hline $46^{-}$ & $67^{-}$ & $75^{19.3}$ & $62^{-}$ & $50^{-}$ \\
\hline
\end{tabular}




\begin{tabular}{|c|c|c|c|c|c|c|}
\hline Group No. & 1 & 2 & 3 & 4 & 5 & 6 \\
\hline Avenella flexuosa & $57^{-}$ & $8^{-}$ & $33^{-}$ & $57^{-}$ & $38^{-}$ & $50^{-}$ \\
\hline Ranunculus platanifolius & $7^{-}$ & $54^{-}$ & $50^{-}$ & $54^{21.5}$ & $23^{-}$ &.$^{-}$ \\
\hline Solidago virgaurea & $36^{-}$ & $15^{-}$ & $17^{-}$ & $43^{22.1}$ & $23^{-}$ &.$^{-}$ \\
\hline Athyrium distentifolium &.$^{-}$ &.$^{-}$ & $17^{-}$ & $39^{14.3}$ & $46^{-}$ & $50^{-}$ \\
\hline Senecio subalpinus & $14^{-}$ & $31^{-}$ & $17^{-}$ & $39^{20.2}$ & $8^{-}$ & $17^{-}$ \\
\hline Lilium martagon & $43^{-}$ & $38^{-}$ & $33^{-}$ & $14^{-}$ & $8^{-}$ &.$^{-}$ \\
\hline Thalictrum aquilegiifolium & $7^{-}$ & $23^{-}$ & $50^{-}$ & $39^{21.7}$ &.$^{-}$ &.$^{-}$ \\
\hline Paris quadrifolia & $14^{-}$ & $15^{-}$ & $17^{-}$ & $36^{23.6}$ & $15^{-}$ & $\cdot-$ \\
\hline Ranunculus lanuginosus & $\cdot^{-}$ & $23^{-}$ & $50^{-}$ & $32^{15.2}$ & $8^{-}$ &.$^{-}$ \\
\hline Moneses uniflora & $29^{-}$ & $8^{-}$ & $17^{-}$ & $18^{-}$ & $31^{-}$ &.$^{-}$ \\
\hline Acetosa arifolia &.$^{-}$ & $15^{-}$ & $\cdot^{-}$ & $29^{-}$ & $8^{-}$ & $50^{-}$ \\
\hline Alchemilla sp. div. &.$^{-}$ &.$^{-}$ &.$^{-}$ & $36^{24.9}$ & $8^{-}$ & $50^{-}$ \\
\hline Gymnocarpium dryopteris & $21^{-}$ & $31^{-}$ &.$^{-}$ & $21^{-}$ &.$^{-}$ &.$^{-}$ \\
\hline Cystopteris fragilis &.$^{-}$ & $8^{-}$ & $33^{-}$ & $25^{20.0}$ &.$^{-}$ &.$^{-}$ \\
\hline Hypericum maculatum &.$^{-}$ &.$^{-}$ & $17^{-}$ & $18^{-}$ & $8^{-}$ & $33^{-}$ \\
\hline Pulmonaria obscura &.$^{-}$ & $23^{-}$ & $17^{-}$ & $14^{-}$ &.$^{-}$ &.$^{-}$ \\
\hline Silene dioica &.$^{-}$ & $23^{-}$ & $17^{-}$ & $14^{-}$ &.$^{-}$ &.$^{-}$ \\
\hline Valeriana sambucifolia &.$^{-}$ & $8^{-}$ & $33^{-}$ & $18^{-}$ &.$^{-}$ &.$^{-}$ \\
\hline Huperzia selago & $\cdot^{-}$ & $8^{-}$ & $17^{-}$ & $21^{23.2}$ &.$^{-}$ &.$^{-}$ \\
\hline Cardamine impatiens &.$^{-}$ &.$^{-}$ & $17^{-}$ & $14^{-}$ & $23^{-}$ & $\cdot-$ \\
\hline Petasites albus & $14^{-}$ & $8^{-}$ & $17^{-}$ & $11^{-}$ &.$^{-}$ &.$^{-}$ \\
\hline Urtica dioica & $\cdot-$ &.$^{-}$ & $17^{-}$ & $18^{14.9}$ &.$^{-}$ & $17^{-}$ \\
\hline Cardaminopsis halleri &.$^{-}$ &.$^{-}$ &.$^{-}$ & $14^{-}$ & $23^{-}$ &.$^{-}$ \\
\hline Phegopteris connectilis & $\cdot^{-}$ &.$^{-}$ & $17^{-}$ & $11^{-}$ &.$^{-}$ & $33^{-}$ \\
\hline Anthriscus nitidus &.$^{-}$ &.$^{-}$ & $33^{-}$ & $14^{-}$ &.$^{-}$ &.$^{-}$ \\
\hline Asarum europaeum &.$^{-}$ & $8^{-}$ & $17^{-}$ & $4^{-}$ & $15^{-}$ &.$^{-}$ \\
\hline Poa nemoralis &.$^{-}$ &.$^{-}$ & $17^{-}$ & $7^{-}$ & $8^{-}$ &.$^{-}$ \\
\hline Angelica sylvestris &.$^{-}$ &.$^{-}$ &. & $7^{-}$ & $15^{-}$ &.$^{-}$ \\
\hline Hieracium prenanthoides &.$^{-}$ & $8^{-}$ & $17^{-}$ & $4^{-}$ &.$^{-}$ &.- \\
\hline Aegopodium podagraria &.$^{-}$ & $8^{-}$ &.$^{-}$ & $7^{-}$ &.$^{-}$ &.$^{-}$ \\
\hline Coeloglossum viride &.$^{-}$ & $8^{-}$ &.$^{-}$ & $7^{-}$ &.$^{-}$ &.$^{-}$ \\
\hline Geranium phaeum & $\cdot^{-}$ & $\cdot^{-}$ & $17^{-}$ &.$^{-}$ & $15^{-}$ &.$^{-}$ \\
\hline Lunaria rediviva & $\cdot^{-}$ &.$^{-}$ & $17^{-}$ & $7^{-}$ &. &.$^{-}$ \\
\hline Festuca picturata &.$^{-}$ &.$^{-}$ & $17^{-}$ & $7^{-}$ &.$^{-}$ &.$^{-}$ \\
\hline Potentilla aurea &.$^{-}$ &.$^{-}$ & $\cdot-$ & $7^{-}$ & $\cdot-$ & $17^{-}$ \\
\hline Aruncus dioicus &.$^{-}$ &.$^{-}$ &.$^{-}$ & $7^{-}$ & $8^{-}$ &.$^{-}$ \\
\hline Tussilago farfara & $\cdot^{-}$ &.$^{-}$ &.$^{-}$ & $7^{-}$ &.$^{-}$ & $17^{-}$ \\
\hline Circaea alpina &.$^{-}$ &.$^{-}$ &.$^{-}$ & $4^{-}$ & $15^{-}$ &.$^{-}$ \\
\hline Adoxa moschatellina &.$^{-}$ &.$^{-}$ &.$^{-}$ & $4^{-}$ & $15^{-}$ &.$^{-}$ \\
\hline Convallaria majalis & $7^{-}$ &.$^{-}$ & $17^{-}$ &.$^{-}$ &.$^{-}$ &.$^{-}$ \\
\hline Galium odoratum &.$^{-}$ & $8^{-}$ &.$^{-}$ &.$^{-}$ &.$^{-}$ & $17^{-}$ \\
\hline Campanula rapunculoides & $\cdot-$ & $8^{-}$ & $17^{-}$ & $\cdot-$ &.$^{-}$ &.$^{-}$ \\
\hline Stachys alpina & $\cdot^{-}$ &.$^{-}$ & $17^{-}$ & $4^{-}$ &.$^{-}$ &.$^{-}$ \\
\hline Hylotelephium argutum & $\cdot^{-}$ & $\cdot^{-}$ & $17^{-}$ & $4^{-}$ & $\cdot-$ &.$^{-}$ \\
\hline Ajuga reptans &.$^{-}$ &.$^{-}$ & $17^{-}$ & $4^{-}$ &.$^{-}$ &.$^{-}$ \\
\hline Galeopsis speciosa &.$^{-}$ &.$^{-}$ & $17^{-}$ &.$^{-}$ &.$^{-}$ & $17^{-}$ \\
\hline Polygonatum odoratum & $\cdot-$ &.$^{-}$ & $17^{-}$ & $4^{-}$ &.$^{-}$ &.$^{-}$ \\
\hline
\end{tabular}




\begin{tabular}{|c|c|c|c|c|c|c|c|}
\hline Group No. & & 1 & 2 & 3 & 4 & 5 & 6 \\
\hline Dentaria bulbifera & &.$^{-}$ &.$^{-}$ & $17^{-}$ & $4^{-}$ &.$^{-}$ &.$^{-}$ \\
\hline Phleum rhaeticum & &.$^{-}$ &.$^{-}$ &.$^{-}$ &.$^{-}$ & $8^{-}$ & $17^{-}$ \\
\hline \multicolumn{8}{|c|}{ Diagnostic ground layer species $\left(\mathrm{E}_{0}\right)$} \\
\hline Ctenidium molluscum & *** & $79^{56.1}$ & $31^{-}$ & $17^{-}$ & $21^{-}$ &.$^{-}$ &.$^{-}$ \\
\hline Mnium spinosum & *** & $93^{55.7}$ & $8^{-}$ & $33^{-}$ & $46^{-}$ & $23^{-}$ &.$^{-}$ \\
\hline Pleurozium schreberi & ** & $\mathbf{5 0}^{48.4}$ & $15^{-}$ & $\cdot^{-}$ & $14^{-}$ &.$^{-}$ &.$^{-}$ \\
\hline Dicranum scoparium & *** & $100^{43.2}$ & $31^{-}$ & $33^{-}$ & $64^{-}$ & $15^{-}$ & $67^{-}$ \\
\hline Rhytidiadelphus loreus & * & $14^{34.9}$ &.$^{-}$ & $\cdot^{-}$ &.$^{-}$ &.$^{-}$ &.$^{-}$ \\
\hline Tortella tortuosa & ** & $64^{34.9}$ & $54^{24.6}$ & $33^{-}$ & $14^{-}$ & $8^{-}$ &.$^{-}$ \\
\hline Plagiothecium curvifolium & ** & $64^{31.9}$ & $15^{-}$ &.$^{-}$ & $18^{-}$ & $23^{-}$ & $67^{-}$ \\
\hline Hylocomium splendens & * & $57^{29.5}$ & $31^{-}$ & $33^{-}$ & $21^{-}$ & $23^{-}$ &.$^{-}$ \\
\hline Plagiochila asplenioides & * & $36^{25.7}$ & $23^{-}$ & $17^{-}$ &.$^{-}$ & $15^{-}$ &.$^{-}$ \\
\hline Cirriphyllum tommasinii & * &.$^{-}$ & $15^{36.3}$ &.$^{-}$ &.$^{-}$ &.$^{-}$ &.$^{-}$ \\
\hline Eurhynchium angustirete & ** &.$^{-}$ & $8^{-}$ & $50^{56.5}$ & $7^{-}$ &.$^{-}$ &.$^{-}$ \\
\hline Hypnum cupressiforme & ** &.$^{-}$ &.$^{-}$ & $33^{54.2}$ &.$^{-}$ &.$^{-}$ &.$^{-}$ \\
\hline Plagiothecium laetum & * &.$^{-}$ &.$^{-}$ & $33^{50.6}$ & $4^{-}$ &.$^{-}$ &.$^{-}$ \\
\hline Conocephalum conicum & ** &.$^{-}$ &.$^{-}$ &.$^{-}$ & $21^{43.0}$ &.$^{-}$ &.$^{-}$ \\
\hline Cirriphyllum piliferum & ** &.$^{-}$ &.$^{-}$ &.$^{-}$ & $21^{43.0}$ &.$^{-}$ &.$^{-}$ \\
\hline Rhytidiadelphus squarrosus & * &.$^{-}$ &.$^{-}$ &.$^{-}$ & $14^{34.9}$ &.$^{-}$ &.$^{-}$ \\
\hline Barbilophozia lycopodioides & * &.$^{-}$ &.$^{-}$ &.$^{-}$ & $11^{30.2}$ &.$^{-}$ &.$^{-}$ \\
\hline Drepanocladus uncinatus & * &.$^{-}$ &.$^{-}$ &.$^{-}$ & $11^{30.2}$ &.$^{-}$ &.$^{-}$ \\
\hline Fissidens taxifolius & * &.$^{-}$ &.$^{-}$ &.$^{-}$ & $11^{30.2}$ &.$^{-}$ &.$^{-}$ \\
\hline Plagiochila porelloides & * &.$^{-}$ &.$^{-}$ &.$^{-}$ & $11^{30.2}$ &.$^{-}$ &.$^{-}$ \\
\hline Brachythecium salebrosum & * &.$^{-}$ &.$^{-}$ &.$^{-}$ & $11^{30.2}$ &.$^{-}$ &.$^{-}$ \\
\hline Sphagnum girgensohnii & * &.$^{-}$ &.$^{-}$ &.$^{-}$ & $4^{-}$ & $23^{40.5}$ &.$^{-}$ \\
\hline Plagiothecium cavifolium & * &.$^{-}$ &.$^{-}$ &.$^{-}$ &.$^{-}$ & $15^{36.3}$ &.$^{-}$ \\
\hline Mnium spinulosum & * &.$^{-}$ &.$^{-}$ &.$^{-}$ &.$^{-}$ & $15^{36.3}$ &.$^{-}$ \\
\hline Polytrichum formosum & *** & $7^{-}$ &.$^{-}$ &.$^{-}$ & $21^{-}$ & $15^{-}$ & $83^{68.0}$ \\
\hline Pellia sp. & *** &.$^{-}$ & $\cdot^{-}$ &.$^{-}$ &.$^{-}$ & $\cdot^{-}$ & $50^{67.4}$ \\
\hline Polytrichum commune & ** &.$^{-}$ &.$^{-}$ &.$^{-}$ &.$^{-}$ &.$^{-}$ & $33^{54.2}$ \\
\hline \multicolumn{8}{|c|}{ Other ground layer species $\left(\mathrm{E}_{0}\right)$} \\
\hline Rhytidiadelphus triquetrus & & $36^{-}$ & $38^{-}$ & $17^{-}$ & $21^{-}$ &.$^{-}$ &.$^{-}$ \\
\hline Rhizomnium punctatum & & $\cdot^{-}$ & $23^{-}$ & $33^{-}$ & $11^{-}$ & $8^{-}$ &.$^{-}$ \\
\hline Brachythecium velutinum & &.$^{-}$ &.$^{-}$ &.$^{-}$ & $11^{-}$ & $23^{-}$ &.$^{-}$ \\
\hline Blepharostoma trichophyllum & &.$^{-}$ &.$^{-}$ & $17^{-}$ & $14^{18.5}$ &.$^{-}$ &.$^{-}$ \\
\hline Fissidens dubius & &.$^{-}$ & $15^{-}$ & $17^{-}$ & $4^{-}$ &.$^{-}$ &.$^{-}$ \\
\hline Atrichum undulatum & &.$^{-}$ & $8^{-}$ &.$^{-}$ &.$^{-}$ & $15^{-}$ & $17^{-}$ \\
\hline Plagiothecium denticulatum & &.$^{-}$ &.$^{-}$ &.$^{-}$ & $7^{-}$ & $15^{-}$ &.$^{-}$ \\
\hline Lepidozia reptans & & $7^{-}$ & $15^{-}$ &.$^{-}$ &.$^{-}$ &.$^{-}$ &.$^{-}$ \\
\hline Cladonia coniocraea & &.$^{-}$ &.$^{-}$ & $17^{-}$ & $7^{-}$ &.$^{-}$ &.$^{-}$ \\
\hline Dicranella heteromalla & &.$^{-}$ &.$^{-}$ & $17^{-}$ & $4^{-}$ &.$^{-}$ & $17^{-}$ \\
\hline Plagiomnium affine & &.$^{-}$ &.$^{-}$ & $17^{-}$ &.$^{-}$ & $15^{-}$ &.$^{-}$ \\
\hline Plagiomnium cuspidatum & &.$^{-}$ &.$^{-}$ &.$^{-}$ & $7^{-}$ & $8^{-}$ &.$^{-}$ \\
\hline Tetraphis pellucida & &.$^{-}$ &.$^{-}$ &.$^{-}$ & $7^{-}$ & $8^{-}$ &.$^{-}$ \\
\hline Calypogeia azurea & &.$^{-}$ & $8^{-}$ &.$^{-}$ &.$^{-}$ &.$^{-}$ & $17^{-}$ \\
\hline Plagiomnium undulatum & &.$^{-}$ &.$^{-}$ & $17^{-}$ & $4^{-}$ &.$^{-}$ &.$^{-}$ \\
\hline Dicranum montanum & &.$^{-}$ &.$^{-}$ &.$^{-}$ & $4^{-}$ & $8^{-}$ &.$^{-}$ \\
\hline Plagiomnium rostratum & &.$^{-}$ &.$^{-}$ &.$^{-}$ &.$^{-}$ & $8^{-}$ & $17^{-}$ \\
\hline
\end{tabular}




\section{Seslerio caeruleae-Piceetum abietis Fajmonová 1978 nom. corr. et nom. cons. propos.}

Nomenclatural type: Fajmonová (1978), tab. 1, rel. 6, holotype.

Original name: Seslerio-Piceetum (Fajmonová, 1978, p. 553), i.e. Seslerio variae-Piceetum abietis Fajmonová 1978 nom. inept. (Rec. 10C, Art. 44)

Non: Seslerio variae-Piceetum Eggler 1952, Seslerio-Piceetum Zukrigl 1973 nom. inval. (Art. 3b)

Set of the most important diagnostic species:

$\mathrm{E}_{1}$ : Ranunculus breyninus, Campanula cochleariifolia, Phyteuma orbiculare, Crepis jacquinii, Vaccinium vitisidaea, Carex ornithopoda, Carex digitata, ${ }^{*}$ Sesleria caerulea, *Bellidiastrum michelii, Carex sempervirens ssp. tatrorum, Carduus glaucinus, Swertia perennis, Tofieldia calyculata, Maianthemum bifolium, Clematis alpina, Calamagrostis varia, Poa alpina, *Pimpinella major, Festuca tatrae, Orthilia secunda, Campanula rotundifolia agg., ${ }^{*}$ Cardaminopsis arenosa agg., *Calamagrostis arundinacea.

Relevé data: Fajmonová (1978), tab. 1, rels. 1-13; Fajmonová (1986), tab. 1, rel. 5; see fig. 3 .

The association was described for Western Carpathian natural supramontane (marginally also upper montane) Norway spruce phytocoenoses on the most extreme habitats developed over dolomites and limestones within the class Vaccinio-Piceetea (Fajmonová, 1978). Their canopy is considerably open (cover 50-70 [80]\%), formed by dominant Picea abies and admixed Sorbus aucuparia. Other tree species could also participate in canopy species composition: Acer pseudoplatanus, Larix decidua, Sorbus aria and sparsely shrubby Fagus sylvatica. In the understorey are usually growing Daphne mezereum, Lonicera nigra, Pinus mugo, Rosa pendulina, exceptionally also Salix silesiaca.

High species diversity of the field layer along with considerable number of the highly constant species is a characteristic feature of the community stands. The field layer dominant is Sesleria caerulea, here and there mutually with Calamagrostis varia. The group of calcicoles has significant abundance (Bellidiastrum michelii, Carduus glaucinus, Cirsium erisithales, Cortusa matthioli, Phyteuma orbiculare etc.), including the species which indicate shallow soils or stony habitats (Asplenium viride, Cardaminopsis arenosa agg., Carex sempervirens ssp. tatrorum, Crepis jacquinii, Festuca tatrae, Poa alpina, Ranunculus breyninus, Tofieldia calyculata). Substantial importance has the group of species which in the higher mountain altitudes prefer habitats on calcareous rocks (Astrantia major, Clematis alpina, Carex digitata, Crepis paludosa, Fragaria vesca, Heracleum sphondylium, Geranium sylvaticum, Mercurialis perennis, Phyteuma spicatum, Polygonatum verticillatum, Primula elatior, Valeriana tripteris and many others).

With high constancy in the stands are also growing Calamagrostis arundinacea, Maianthemum bifolium, Luzula sylvatica ssp. sylvatica, Soldanella marmarossiensis agg., Prenanthes purpurea, Oxalis acetosella or Homogyne alpina, Vaccinium myrtillus, V. vitis-idaea, Avenella flexuosa. Species as Viola biflora, Adenostyles alliariae, Melampyrum sylvaticum, Senecio nemorensis agg. (Fajmonová 1978 ut S. *jacquinianus and S. fuchsii), Leucanthemum rotundifolium also frequently participate in the species composition of the phytocoenoses.

The most frequent ground layer species are Ctenidium molluscum, Dicranum scoparium, Mnium spinosum, Plagiothecium curvifolium and Tortella tortuosa. With a lower constancy are present e.g. Hylocomium splendens and Pleurozium schreberi.

Variability. - Due to low total number of recorded relevés, variability of the association Seslerio caeruleae-Piceetum Fajmonová 1978 is still insufficiently known. Therefore only variants are syntaxonomically recognized here:

(1) Aquilegia vulgaris-variant (diferential species: Aquilegia vulgaris, Gentiana asclepiadea, Moneses uniflora, Pimpinella major, Tofieldia calyculata, data: Fajmonová (1978), tab. 1. rel. 1, 2, 6-10);

(2) Pyrethrum clusii-variant (diferential species: $G a$ lium schultesii, Lilium martagon, Melampyrum sylvaticum, Poa stiriaca, Pyrethrum clusii, Rubus saxatilis; data: Fajmonová 1978, tab. 1. rel. 4, 5, 12, 13, Fajmonová (1986), tab. 1. rel. 5);

(3) Paris quadrifolia-variant (differential species Dentaria enneaphyllos, Dryopteris dilatata, Galeobdolon luteum agg., Lathyrus vernus, Paris quadrifolia, data: Fajmonová (1978), tab. 1. rel. 3, 11) with differential species indicating less extreme habitat conditions, however, dominance of Sesleria caerulea is maintained.

Nomenclatural note. - According to the taxonomical reassessments in the genus Sesleria (Foggi et al., 2001), the name Sesleria varia (Jacq.) Wettst. used by Fajmonová (1978) is a later synonym of the correct name Sesleria caerulea (L.) Ard. Since the name S. caerulea (L.) Ard. is accepted in the newer botanical literature (Kubát et al., 2002; Fischer et al., 2008; Tisson et al., 2014; Jäger et al., 2017; Kaplan et al., 2019; Mered'a et al., 2019; etc.), formal change of the original name Seslerio variae-Piceetum Fajmonová 1978 is here proposed.

The name Seslerio variae-Piceetum Fajmonová 1978 is a later homonym of the validly published name Seslerio 
variae-Piceetum Eggler 1952 (see also the syntaxonomical note). However, the latter name was never accepted by later Austrian authors (Wallnöfer, 1993, p. 320; Exner, 2007, p. 191; Willner, 2007, p. 238) and Willner (2007, p. 238) proposed to give the nomenclatural priority to its later syntaxonomical synonym Adenostylo glabrae-Piceetum Zukrigl 1973. Recent Slovenian authors seem to accept the name "Seslerio variae-Piceetum Eggler 1952", though only one relevé is known from Slovenia (Zupančič, 1999, tab. 17) and it represent floristically and ecologically different community from Seslerio variae-Piceetum Eggler 1952; according to Zupančič the phytocoenosis represent a secondary Norway spruce community (cf. Šilc \& Čarni, 2012; Juvan et al., 2013).

On the contrary, the name Seslerio variae-Piceetum Fajmonová 1978 was immediately accepted in the Slovak literature for the respective natural supramontane(montane) Norway spruce community (Šomšák in $\mathrm{Mu}-$ cina et al., 1985) and was continuously used to the present (Jarolímek et al., 2008a; Kučera, 2010a; Kučera,

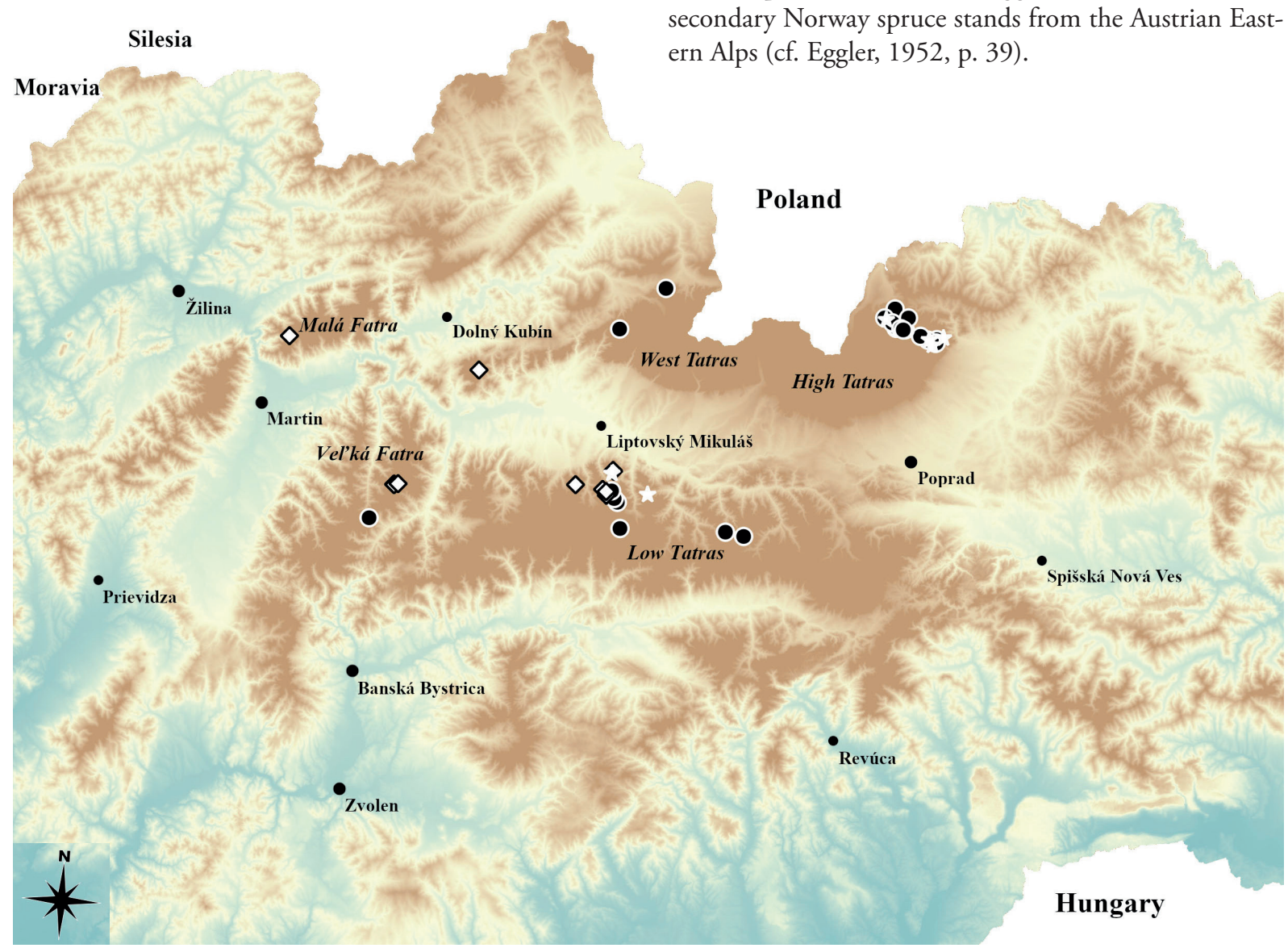

2012a). To avoid an inconvenient rejection of this commonly used name, it is formally proposed for protection as a nomen conservandum (cf. Kučera, 2012a, p. 210), with consideration of the required nomenclatural change to Seslerio caerulae-Piceetum Fajmonová 1978 nom. corr.

Syntaxonomical note. - Phytocoenoses documented by Eggler (1952) and Zupančič (1999) does not belong to Seslerio caerulae-Piceetum Fajmonová 1978 due to a difspecies Adenostyles glabra, Galium verum, Pulmonaria stiriaca, Soldanella alpina, different abundances of Fragaria vesca, Poa stiriaca, Oxalis aceotsella, valeriana tripteris etc. as recorded by Eggler; or Erica carnea, Cyclamen purpurascens, Helleborus niger ssp. niger, Polygala chamaebuxus etc. recorded by Zupančič). Moreover, the phytocoenoses of Fajmonová (1978) represent ecologically most extreme natural calcicolous Norway spruce community of the Western Carpathians, while the relevés of SeslerioPiceetum poetosum stiriacae of Eggler (1952) reflect mostly secondary Norway spruce stands from the Austrian Eastern Alps (cf. Eggler, 1952, p. 39). ferent phytochorological and/or ecological content (e.g.

Figure 2: Distribution of analysed associations' relevés in Slovakia: diamonds - Cirsio erisithalis-Piceetum, stars - Fragario vescae-Piceetum, circles Adenostylo alliariae-Piceetum. Made with QGIS.

Slika 2: Razširjenost popisov obravnavanih asociacij na Slovaškem: diamanti - Cirsio erisithalis-Piceetum, zvezde - Fragario vescae-Piceetum, krogi Adenostylo alliariae-Piceetum. Narejeno z QGIS. 


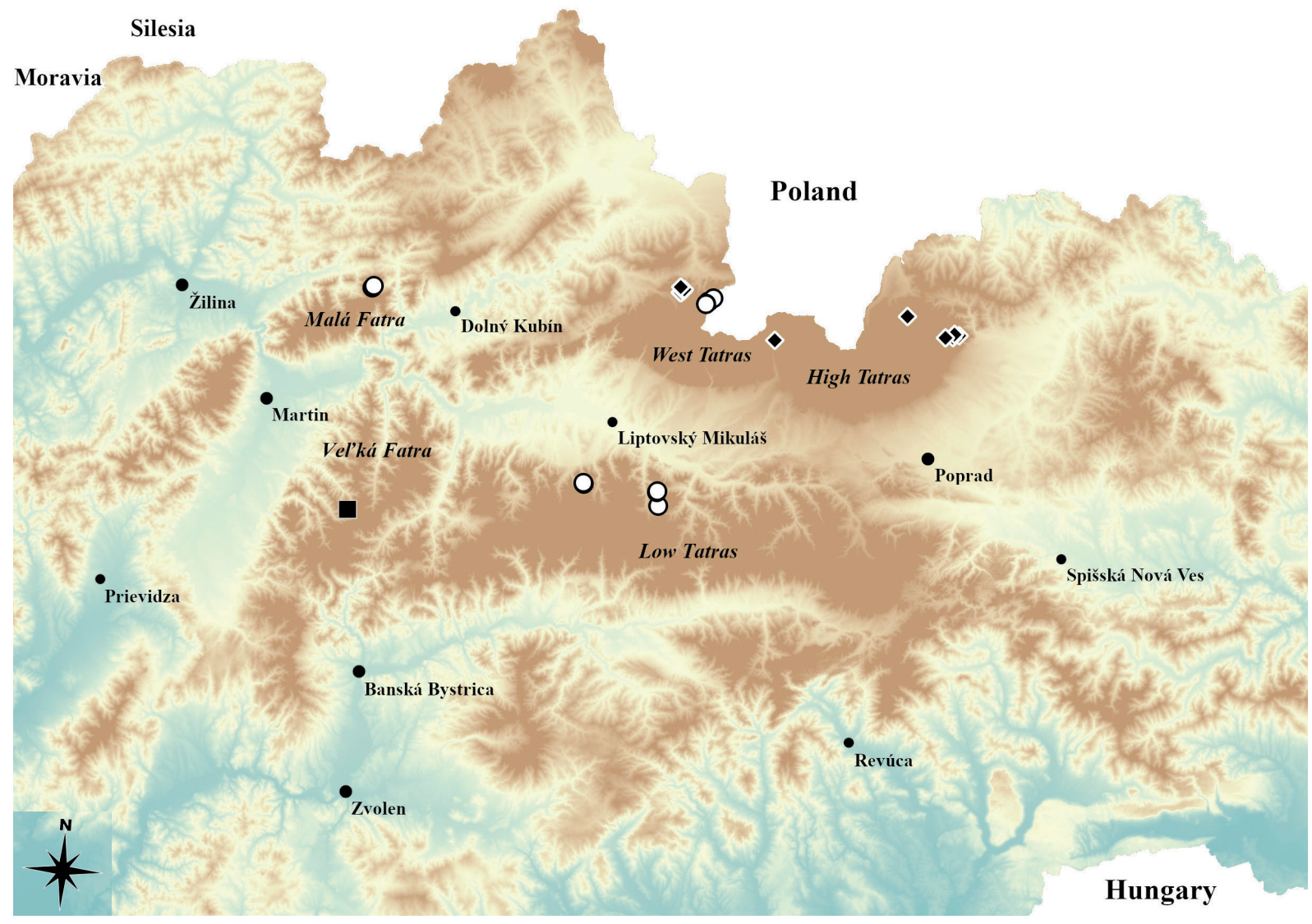

Figure 3: Distribution of analysed associations' relevés in Slovakia: circles - Seslerio caeruleae-Piceetum, diamonds - Mnio spinosi-Piceetum, squares (overlapping) - Hieracio murorum-Piceetum. Made with QGIS.

Slika 3: Razširjenost popisov obravnavanih asociacij na Slovaškem: krogi - Seslerio caeruleae-Piceetum, diamanti - Mnio spinosi-Piceetum, kvadrati (se prekrivajo) - Hieracio murorum-Piceetum. Narejeno z QGIS.

\section{Cirsio erisithalis-Piceetum abietis}

\section{Fajmonová et P. Kučera ass. nov. hoc loco}

Nomenclatural type: Fajmonová (1986), tab. 1, rel. 11, holotypus hoc loco.

Incl.: Piceetum excelsae altherbosum calcicolum Sillinger 1933 subtyp Oxalis p. p., Sorbo-Piceetum calcicolum Šmarda et al. 1971 facies calamagrostietosum p. p. min., Cortuso-Piceetum calamagrostietosum variae sensu Fajmonová 1986 non (Šoltés 1976) Fajmonová 1986, Cortuso-Piceetum typicum (sensu Fajmonová 1986), Cortuso-Piceetum saxifragetosum rotundifolii Fajmonová 1986, Cortuso-Piceetum adenostyletosum alliariae Fajmonová 1986

Pseud.: Cortuso-Piceetum sensu Fajmonová 1978 non (Šoltés 1976) Fajmonová 1978 p. p. min., Cortuso-Piceetum sensu Fajmonová 1986 non (Šoltés 1976) Fajmonová 1978

Non: Piceetum excelsae normale calcicolum Sillinger 1933 nom. illeg. (Art. 34a), Adenostylo alliariae-Piceetum cortusetosum Šoltés 1976
Set of the most important diagnostic species:

$\mathrm{E}_{1}$ : Cystopteris montana, Dentaria enneaphyllos, ${ }^{*}$ Cardaminopsis arenosa agg., Rubus saxatilis, Festuca carpathica, Asplenium viride, Ranunculus nemorosus, Laserpitium latifolium, ${ }^{*}$ Sesleria caerulea, Allium victorialis, ${ }^{*}$ Tithymalus amygdaloides, ${ }^{*}$ Bellidiastrum michelii, *Chaerophyllum hirsutum, ${ }^{*}$ Galium schultesii, ${ }^{*}$ Pimpinella major.

Relevé data and original diagnosis: Fajmonová (1986), tab. 1, rels. 1-4, 11, 13, 15-17, 21, 23-25; see fig. 2.

Supramontane Picea abies woodland of less extreme habitats of limestones and dolomites (in comparison to Seslerio-Piceetum Fajmonová 1978), however, the slopes are still steep ([15] 25-30 $)$ and, consequently, canopy cover is considerably open (55-75 [80]\%). Sorbus aucuparia is a constant companion of Norway spruce, admixed tree species are Acer pseudoplatanus (often), S. aria (occasionally) and Fagus sylvatica (only exceptionally and then with a low growth). Daphne mezereum is a constant species of the understorey, less frequently is growing Salix 
silesiaca, here and there are present also Pinus mugo, Lonicera nigra and Ribes petraeum.

The field layer is usually dominated by Cortusa matthioli, frequently accompanied by Luzula sylvatica ssp. sylvatica. Habitats with moderately shallow but very skeleton-rich soils, for example rocky slopes and ridges, induce opening of canopy cover and Calamagrostis varia dominates in the field cover. An ecological opposite constitutes the sub-community with Adenostyles alliariae or Saxifraga rotundifolia as codominant species (cover up to ca. 25\%) to Cortusa.

Equally as in the case of Seslerio-Piceetum Fajmonová 1978 , very species rich composition of this community consists of numerous species with high constancy and many less frequent species. Characteristic features are the group of calcicoles (in addition to Cortusa and Calamagrostis varia for example Cirsium erisithales, Asplenium viride, Cardaminopsis arenosa agg., Sesleria albicans, Bellidiastrum michelii) and very abundant group of species which prefer calcareous soils in higher mountain altitudes (Astrantia major, Crepis paludosa, Galium schultesii, Geranium sylvaticum, Heracleum sphondylium, Hieracium murorum, Phyteuma spicatum, Polygonatum verticillatum, Primula elatior, Valeriana tripteris and others).

The constant components of stands of the association are species commonly growing also in other types of natural Norway spruce communities: Luzula sylvatica ssp. sylvatica, Vaccinium myrtillus, Gentiana asclepiadea, Homogyne alpina, Oxalis acetosella, Prenanthes purpurea, Senecio hercynicus etc. (Kučera, 2012a). In the stands are usually with low cover but frequently growing also Rubus saxatilis, Polystichum lonchitis, Chaerophyllum hirsutum, Tithymalus amygdaloides; Viola biflora, Dentaria enneaphyllos and Mercurialis perennis could here and there reach cover over $5 \%$.

Moss species are absent in some stands of the community. The most frequent ground layer species is Tortella tortuosa, with lower constancy are occurring especially Ctenidium molluscum, Mnium spinosum, Rhizomnium punctatum, along with common woodland moss species Dicranum scoparium, Hylocomium splendens and Rhytidiadelphus triquetrus.

Variability. - In dependence on the variability in habitat ecology and, consequently, field layer species composition as well as physiognomy, a series of sub-communities is differentiated following the soil shallowness and amount of soil skeleton:

(1) subassociation Cirsio erisithalis-Piceetum calamagrostietosum variae Fajmonová et P. Kučera subass. nov. hoc loco (nomenclatural type: Fajmonová (1986), tab. 1, rel. 4, holotypus hoc loco; differential spe- cies: Calamagrostis varia (dominant), Carduus glaucinus, Laserpitium latifolium, Melampyrum sylvaticum, Pimpinella major, Pinus mugo; original diagnosis: Fajmonová 1986, tab. 1, rels. 1-4) on the most extreme habitats within the association (see Fajmonová 1986, p. 50); this subcomunity corresponds to the unit differentiated by Fajmonová (1986) under the pseudonym Cortuso-Piceetum calamagrostietosum variae sensu Fajmonová 1986 non (Šoltés 1976) Fajmonová 1986;

(2) subassociation Cirsio erisithalis-Piceetum typicum subass. nov. hoc loco (nomenclatural type: Fajmonová (1986), tab. 1, rel. 11, holotypus hoc loco, automatical holotype sensu Art. 5b; differential species: Campanula serrata, Geum rivale, Soldanella carpatica; original diagnosis: Fajmonová (1986), tab. 1, rels. 11, 13, $16,17)$ includes ecologically intermediate phytocoenoses within this association; this sub-community corresponds to the unit commonly used under the pseudonym Cortuso-Piceetum typicum sensu Fajmonová 1986 non (Šoltés 1976) Fajmonová 1986 and included are also phytocoenoses of natural Norway spruce woodland classified as Cortuso-Piceetum saxifragetosum rotundifolii Fajmonová 1986;

(3) subassociation Cirsio erisithalis-Piceetum adenostyletosum alliariae (Fajmonová 1986) P. Kučera comb. nov. hoc loco (basionym: Cortuso-Piceetum adenostyletosum alliariae Fajmonová 1986 [Fajmonová, 1986, p. 51]; nomenclatural type: Fajmonová (1986), tab. 1, rel. 24, holotypus [Fajmonová, 1986, p. 51]; differential species: Calamagrostis arundinacea, Chrysosplenium alternifolium, Cicerbita alpina, Clematis alpina, Gymnocarpium dryopteris, Leucanthemum rotundifolium, Poa stiriaca, Ranunculus platanifolius, Soldanella marmarossiensis agg.; Rhizomnium punctatum, Rhytidiadelphus triquetrus; original diagnosis: Fajmonová (1986), tab. 1, rels. 15, 21, 23-25) comprises phytocoenoses developed over slightly deeper calcareous soils, Adenostyles alliariae and Cortusa matthioli are codominant species.

Nomenclatural and syntaxonomical note. - Up to the present, phytocoenoses of this association were included under the association name Cortuso-Piceetum (Šoltés 1976) Fajmonová 1978 (see Fajmonová, 1986). Fajmonová (1978) initially labelled the new association as "Cortuso-Piceetum (Sillinger 1933) Šoltés 1976" because it was based on a subassociation cited by Fajmonová (1976) in the form "Adenostylo-Piceetum cortusetosum (Sillinger 1933) Šoltés 1976” (cf. Šoltés, 1976).

However, Šoltés (1976) did not describe a "CortusoPiceetum" as well as Sillinger (1933) did not describe a subassociation "cortusietosum". Therefore the both names "Cortuso-Piceetum (Sillinger 1933) Šoltés 1976" and 
"Adenostylo-Piceetum cortusetosum (Sillinger 1933) Šoltés 1976" are here considered as nomina ficta (phantom names) and the following author citations are accepted: Cortuso-Piceetum (Šoltés 1976) Fajmonová 1978 and Adenostylo-Piceetum cortusetosum Šoltés 1976.

However, more important are the syntaxonomical differences between the respective original diagnoses of considered units:

(A) Sillinger's (1933) synoptic table of Piceetum excelsae altherbosum calcicolum Sillinger 1933 represent for the most part calcareous secondary Norway spruce forests (with Abies, Acer, Larix or Pinus sylvestris) of the higher montane altitudes (1250-1380 m a.s.l.) of the Low Tatras (cf. Kučera et al., 2009), only two (? three) of in total ten relevés represent a true Vaccinio-Piceetea community; therefore the synoptic table of this Sillinger's unit represent a secondary Norway spruce community of the class Carpino-Fagetea (cf. also the new classification of Slovakian Fagus communities by Ujházyová et al. (2021));

(B) Šoltés (1976, tab. 1 and tab. 3) published a comparative synoptic table of Adenostylo-Piceetum cortusetosum Šoltés 1976 along with 13 original relevés (originally recorded mostly by Lakatosová (1971, tab. 5) [cf. p. 230, 231a]): these original relevés represent for the most part secondary Norway spruce stands developed on habitats of upper montane mixed Fagus sylvatica woodland (cf. Kučera, 2012a) and only negligible part of relevés might be identified with natural supramontane Norway spruce stands - and the corresponding original relevé(s) syntaxonomically belong to the association Adenostylo alliariaePiceetum Samek et al. 1957.

(C) Contrary to the previous two units, Fajmonovás (1986) own relevés mostly represent natural supramontane Norway spruce community (and its sub-communities), which are floristically, ecologically and physiognomically different and, consequently, they represent an independent association floristically related to Seslerio caerulae-Piceetum Fajmonová 1978 nom. corr.

(D) The nomenclatural evaluation based on the Code's Art. $27 \mathrm{~d}$ is that association Cortuso-Piceetum (Šoltés 1976) Fajmonová 1978 have to be interpreted following the original differentiation and original diagnosis of the subassociation Adenostylo alliariae-Piceetum cortusetosum Šoltés 1976, and not according to Fajmonovás own relevés published by Fajmonová (1986) which represent a syntaxonomically different unit from the one published by Šoltés (1976). For this reason, a new association Cirsio erisithalis-Piceetum abietis is here proposed for the three main sub-communities differentiated by Fajmonová (1986) (see Kučera, 2012a).

(E) It migh be argued that the correct author citations should be applied alternatively: "Adenostylo alliariae-Pi- ceetum cortusetosum (Sillinger 1933) Šoltés 1976" and, subsequently, "Cortuso-Piceetum (Sillinger 1933) Fajmonová 1978". It must be reminded that the resulting plant community had to follow Sillinger's (1933) syntaxonomical content and thus it would belong to the class CarpinoFagetea (see above).

\section{Fragario vescae-Piceetum abietis P. Kučera ass. nov. prov.}

Nomenclatural type: Kanka (2008), tab. 19, rel. 3, pro holotypus.

Incl.: Cortuso-Piceetum calamagrostietosum arundinaceae Fajmonová 1986 p. p., Bupleuro longifolii-Laricetum Kanka 2008 ass. prov. p. p. min. (Art. 3b, 3o)

Set of the most important diagnostic species:

$\mathrm{E}_{1}$ : Actaea spicata, Aconitum variegatum, Digitalis grandiflora, Polypodium vulgare, Polystichum aculeatum, Geranium robertianum, * Galium schultesii, *Tithymalus amygdaloides, ${ }^{*}$ Calamagrostis arundinacea.

Relevé data: Fajmonová (1986), tab. 1, rels. 7, 14; Kanka (2008), tab. 17, rels. 2, 10 and tab. 19, rel. 3 and tab. 26, rel. 3; see fig. 2 .

Canopy of this community stands is formed by Picea abies with admixed Acer pseudoplatanus, recorded is also occurrence of Larix decidua, Abies alba and Sorbus aucuparia. Occasional dominance of Larix decidua could indicate a specific successional stage or, eventually, result of the former forest management. Understorey species are Daphne mezereum, Ribes petraeum, Lonicera nigra and Rosa pendulina.

The constant field layer components are for example the species Cirsium erisithales, Epilobium montanum, Fragaria vesca, Galium schultesii, Gentiana asclepiadea, Oxalis acetosella, Primula elatior, Rubus idaeus, Senecio nemorensis agg. (even with cover-abundance over 5\%) and Valeriana tripteris; Calamagrostis arundinacea, eventually Dryopteris filix-mas were here and there observed as distinct dominants. Cortusa matthioli and Mercurialis perennis are subdominant species in some stands.

Among the other considerably frequent species belong Aconitum variegatum, Adenostyles alliariae (with low cover), Astrantia major, Pyrethrum clusii, Ranunculus lanuginosus and other species. In contrast to other calcareous Western Carpathian supramontane Norway spruce plant communities, Vaccinium myrtillus was not recorded till the present.

Occurrence of species Campanula persicifolia, C. trachelium, Digitalis grandiflora within this natural supramontane Picea woodland indicate a nutrient-rich, especially 
lime-rich and considerably drier habitat. Admixture of soil skeleton is indicated by presence of species Actaea spicata, Asplenium viride, Cystopteris fragilis, Hylotelephium argutum, Mercurialis perennis, Polystichum aculeatum, P. lonchitis. Among the low frequent species are also Anthriscus nitidus, Aquilegia vulgaris, Calamagrostis varia, Carex digitata, Delphinium elatum, Lilium martagon, Salvia glutinosa.

The most frequently recorded ground layer species is Eurhynchium angustirete. Other moss species are infrequent, e.g. Dicranum scoparium, Hylocomium splendens, Mnium spinosum, Tortella tortuosa.

Syntaxonomical note. - The association Fragario vescaePiceetum ass. prov. integrates species-rich phytocoenoses which are lacking distinct species of the associations Seslerio caerulae-Piceetum and Cirsio erisithalis-Piceetum (e.g. Bellidiastrum michelii, Pimpinella major, Sesleria albicans) or species bound to one of these units (Carex sempervirens ssp. tatrorum, Cystopteris montana, Phyteuma orbiculare, Ranunculus breyninus and others). On the other side, they do not have tall-herb character of the stands of the association Adenostylo alliariae-Piceetum Samek et al. 1957, even if some of the typical species of the latter unit (for example Adenostyles alliariae) could be present; however, with low cover-abundance values only. However, only six relevés of Fragario vescae-Piceetum prov. are known up to the present.

Typically developed stands are represented by relevés of Kanka (Kanka, 2008; tab. 19, rel. 3 and tab. 26, rel. 3) from the Belianske Tatry Mts, with Digitalis grandiflora accompanied by species Actaea spicata, Campanula persicifolia, C. trachelium, Geranium robertianum, Polystichum aculeatum and Polypodium vulgare.

\section{Adenostylo alliariae-Piceetum abietis} Samek et al. 1957 nom. corr. et nom. cons. propos.

Nomenclatural type: Samek et al. (1957), tab. 14, rel. 54, lectotypus hoc loco.

Original name: Adenostyleto-Piceetum (Samek et al., 1957, p. 15), i.e. Adenostyleto alliariae-Piceetum excelsae Samek et al. 1957 nom. inept. (Art. 30a, 44)

Syntax. syn.: Adenostylo alliariae-Piceetum excelsae Březina et Hadač in Hadač et al. 1969 nom. illeg. (Art. 31), Cortuso-Piceetum (Šoltés 1976) Fajmonová 1978

Incl.: Piceetum excelsae altherbosum calcicolum Sillinger 1933 subtyp nivový p. p., Sorbo-Piceetum calcicolum Šmarda et al. 1971 facies altherbosum, Sorbo-Piceetum calcicolum Šmarda et al. 1971 facies oxalidetosum p. p. min., Adenostylo alliariae-Piceetum cortusetosum Šoltés 1976 p. p. min., Vaccinio myrtilli-Piceetum calamagrostietosum variae Šoltés 1976 (p. p. min.), Cortuso-Piceetum calamagrostietosum variae (Šoltés 1976) Fajmonová 1986

Corresponding nomina ficta (phantom names): Sorbo-Piceetum calcicolum Pawłowski 1956 apud Šmarda et al. 1971 p. p., Cortuso-Piceetum (Sillinger 1933) Šoltés 1976 apud Fajmonová $1978^{11}$ p. p. min.

Non: Adenostylo-Piceetum Hartmann 1953, Adenostylo alliariae-Piceetum Zukrigl 1973 nom. illeg. (Art. 31), Adenostylo alliariae-Piceetum Ellenberg et Klötzli 1974 nom. illeg. (Art. 31)

Set of the most important diagnostic species:

$\mathrm{E}_{1}$ : Doronicum austriacum, Leucanthemum rotundifolium, Milium effusum, Cicerbita alpina, *Stellaria nemorum, ${ }^{*}$ Chaerophyllum hirsutum,

$\mathrm{E}_{0}$ : Conocephalum conicum, Cirriphyllum piliferum.

Relevé data: Šoltés (1969) (msc.), tab. 8, rels. 36, 38; Šmarda et al. (1971), tab. 18, rel. 6; Šoltés (1976), tab. 3, rels. 2, 7 and tab. 4, rel. 35; Kubíček et al. (1992), tab. 1, rel. 4; Černušáková (1994), tab. 2, rel. 14; Miadok (1995), p. 59, rel. 2 and p. 60, rels. 2, 5, 6; Kubíček et al. (1996), p. 90, rel. 1; Kanka (2008), tab. 16, rels. 3, 6, 8, 9 and tab. 17, rels. 1, 3, 5-9, 14; Krajčí (2009) (msc.), tab. 7, rels. 63, 64; Kučera (2012a), p. 319, rel. 101; see fig. 2.

The dominant tree species of the woodland stands of this community is Picea abies, here and there is admixed Sorbus aucuparia (ssp. glabrata) which could temporarily dominate in early successional stages (after a windthrow) (cf. Šoltés, 1969; Šoltés, 1976). Larix decidua was documented only sporadically; however, this tree species was probably more frequent in the regions with its autochthonous occurrence (the Tatra Mountains, some regions of the Low Tatras) before the historical deforestation and other changes of tree species composition. Acer pseudoplatanus and in the lower elevations also Abies alba were originally components of the canopy layer of the stands.

In the understorey shrub species Ribes petraeum and Daphne mezereum very frequently grow, less often also Lonicera nigra, Ribes alpinum, Rosa pendulina, sporadically were recorded Salix silesiaca and S. caprea, and only in the lower limit of natural vertical distribution of this woodland community Fagus sylvatica.

Tall-herb character of the field layer is the physiognomically prominent feature of the stands of this community. Most frequently it is formed by dominant Adenostyles alliariae with other tall forbs (Cicerbita alpina, Doronicum austriacum, Leucanthemum rotundifolium, Senecio nemo-

11 According to J.-P. Theurillat (in e-mail), such phantom name should be cited as follows: "Cortuso-Piceetum (Sillinger 1933) Šoltés 1976 [recte: Cortuso-Piceetum (Šoltés 1976) Fajmonová 1978]”. 
rensis agg.) along with Luzula sylvatica ssp. sylvatica; occassionally Dryopteris dilatata is subdominant species.

Constant species of the lower forb layer are Oxalis acetosella (rarely as dominant), Homogyne alpina, Stellaria nemorum, Soldanella marmarossiensis agg., Ranunculus platanifolius etc. Characteristic is abundant presence of nutrient-demanding species, for example Valeriana tripteris, Myosotis sylvatica, Galeobdolon luteum agg., Polygonatum verticillatum, Epilobium montanum, Primula elatior, Phyteuma spicatum, Viola biflora, Fragaria vesca, Thalictrum aquilegiifolium and others (Kučera, 2012a).

On the contrary to the previous three associations, Athyrium distentifolium is here and there component of the stands (along with $A$. filix-femina). Presence of Vaccinium myrtillus in the stands is reduced. Cortusa matthioli is less frequent, however, here and there it grows with a higher cover (above 5\%).

The most frequent ground layer species are Dicranum scoparium and Mnium spinosum, relatively abundant are also Plagiothecium curvifolium, Conocephalum conicum, Cirriphyllum piliferum, Hylocomium splendens. In phytocoenoses with Sesleria tatrae, Ctenidium molluscum and Polytrichum formosum also belong among the more frequent species.

Variability. - Following four sub-communities could be distinguished according to the floristical and ecological differences:

(1) subassociation Adenostylo-Piceetum typicum (nomenclatural type: Samek et al. (1957), tab. 14, rel. 54, holotypus hoc loco, automatical holotype sensu Art. 5b; differential species: Alchemilla sp. div., Chrysosplenium alternifolium, Galeobdolon luteum agg., Mycelis muralis, Paris quadrifolia, Ranunculus platanifolius, Senecio subalpinus); original diagnosis: Samek et al. (1957), tab. 14, rels. 32, 54, 45, 25; Šmarda et al. (1971), tab. 18, rel. 6; Šoltés (1976), tab. 3, rels. 2, 7 and tab. 4, rel. 35; Kubíček et al. (1992), tab. 1, rel. 4; Kubíček et al. (1996), p. 90, rel. 1; Kanka (2008), tab. 16, rels. 8, 9 and tab. 17, rels. 3, 14; Krajčí (2009) (msc.), tab. 7, rels. 63, 64; Kučera (2012a), p. 319, rel. 101) represents the most frequent type of the community phytocoenoes.

(2) subassociation Adenostylo-Piceetum seslerietosum tatrae P. Kučera subass. nov. hoc loco (nomenclatural type: Kanka (2008), tab. 17, rel. 8, holotypus hoc loco; differential species: Asplenium viride, Cirsium erisithales, Clematis alpina, Huperzia selago, Moneses uniflora, Sesleria tatrae; original diagnosis: Kanka (2008), tab. 17, rels. 5-9) unites open canopy woodland from very steep slopes of the Belianske Tatry Mts (cf. Kanka, 2008, tab. 17).

(3) subassociation Adenostylo-Piceetum lunarietosum redivivae P. Kučera subass. nov. hoc loco (no- menclatural type: Černušáková (1994), tab. 2, rel. 14, holotypus hoc loco; differential species: Lunaria rediviva, Luzula luzuloides, Pleurozium schreberi, Rhodiola rosea; original diagnosis: Šoltés 1969 (msc.), tab. 8, rels. 36, 38 [the relevés are published below, with the consent of $\mathrm{Dr}$ R. Šoltés], Černušáková (1994), tab. 2, rel. 14) contains phytocoenoses developed on ca. debris habitats (Lunaria rediviva), with considerable presence of Sorbus aucupar$i a$ in the canopy (some relevés represent a successional stage), eventually with Pinus mugo.

Šoltés (1969), tab. 8, rel. 36: Belianske Tatry Mts, Rakúsky chrbát, steep slope to the Dolina Siedmich prameňov, $1440 \mathrm{~m}$ a.s.l., slope aspect: $\mathrm{N}$, slope inclination: $45^{\circ}$, debris background, plot size $20 \times 20 \mathrm{~m}^{2}$, cover $\mathrm{E}_{3}: 40 \%, \mathrm{E}_{2}: 30 \%, \mathrm{E}_{1}: 100 \%, \mathrm{E}_{0}: 60 \%$, stand of young rowan trees (25 yrs. old) , R. Šoltés, 12. 8. 1969:

$\mathrm{E}_{3}$ : Sorbus aucuparia ssp. glabrata f. glabrata 2, Salix silesiaca 1 ,

$\mathrm{E}_{2}$ : Pinus mugo 2, Picea abies 1, Salix silesiaca 1, Betula carpatica +

$\mathrm{E}_{1}$ : Lonicera nigra + , Picea abies + , Ribes uva-crispa,$+ \mathrm{Sa}$ lix silesiaca +, Sorbus aucuparia [ssp. glabrata f. glabrata +],

Avenella flexuosa 2, Oxalis acetosella 2, Chamerion angustifolium 2, Vaccinium myrtillus 2, Adenostyles alliariae 1, Calamagrostis varia 1, Luzula luzuloides 1, Soldanella marmarossiensis agg. 1 [ut S. montana ssp. hungarica], Vaccinium vitis-idaea 1, Athyrium filix-femina +, Campanula tatrae [ut C. rotundifolia] +, Doronicum austriacum + , Dryopteris carthusiana,+ D. flix-mas + , Fragaria vesca +, Galium schultesii +, Homogyne alpina +, Gentiana asclepiadea + , Hieracium murorum + , Hypericum maculatum +, Moneses uniflora +, Myosotis sylvatica +, Orthilia secunda + , Potentilla aurea + , Rubus idaeus + , Senecio ovatus + , Thymus pulegioides +, Cicerbita alpina $\mathrm{r}$, Hieracium lachenalii $\mathrm{r}$, Leucanthemum rotundifolium $\mathrm{r}$, Rhodiola rosea $\mathrm{r}$, Valeriana tripteris $\mathrm{r}$,

$\mathrm{E}_{0}$ : Pleurozium schreberi 3, Dicranum scoparium 2, $H y$ locomium splendens 1, Rhytidiadelphus triquetrus 1, Barbilophozia lycopodioides +, Blepharostoma trichophyllum +, Eurhynchium sp. +, Sanionia uncinata +, Plagiothecium curvifolium +, Polytrichum formosum +, Ptilidium pulcherrimum + .

Šoltés (1969), tab. 8, rel. 38: Belianske Tatry Mts, under Kozí chrbát, approximately $10 \mathrm{~m}$ to the right from the [former] hiking trail towards Skalné vráta, $1420 \mathrm{~m}$ a.s.l., slope aspect: NE, slope inclination: $35^{\circ}$, debris background, plot size $20 \times 20 \mathrm{~m}$, cover $\mathrm{E}_{3}: 75 \%, \mathrm{E}_{2}: 3 \%$, $\mathrm{E}_{1}: 50 \%, \mathrm{E}_{0}: 5 \%$, stand of old rowan trees $(80 \mathrm{yrs}$. old) with other tree species admixed, R. Šoltés 30. 9. 1969:

$\mathrm{E}_{3}$ : Sorbus aucuparia ssp. glabrata f. glabrata 3, Acer pseudoplatanus 1, Larix decidua 1, Picea abies 1,

$\mathrm{E}_{2}$ : Ribes petraeum 1 , 
$\mathrm{E}_{1}$ : Daphne mezereum + , Ribes petraeum,+ R. uva-crispa + , Lonicera nigra $\mathrm{r}$,

Oxalis acetosella 2, Asarum europaeum 1, Calamagrostis varia 1 , Cicerbita alpina 1 , Lunaria rediviva 1 , Luzula luzuloides 1, Myosotis sylvatica 1, Athyrium filix-femina +, Dryopteris filix-mas +, Epilobium montanum +, Fragaria vesca +, Aegopodium podagraria +, Galium schultesii +, Gentiana asclepiadea +, Hypericum maculatum + , Lamium maculatum +, Oreogeum montanum +, Poa nemoralis +, Polygonatum verticillatum + , Pulmonaria obscura,$+ R a-$ nunculus lanuginosus +, Rhodiola rosea +, Rubus idaeus +, Senecio ovatus +, Stellaria nemorum +, Thalictrum aquilegiifolium + , Urtica dioica +,

$\mathrm{E}_{0}$ : Pleurozium schreberi 1, Blepharostoma trichophyllum +, Dicranum montanum +, Plagiothecium curvifolium +, Tetraphis pellucida + .

(4) subassociation Adenostylo-Piceetum stellarietosum nemorum P. Kučera subass. nov. hoc loco (nomenclatural type: Kanka (2008), tab. 17, rel. 1, holotypus hoc loco; without differential species; original diagnosis: Šoltés (1976), tab. 3, rel. 7; Miadok (1995), p. 59, rel. 2 and p. 60, rels. 2, 5, 6; Kanka (2008), tab. 16, rels. 3, 6 and tab. 17 , rel. 1) differentiates by less numerous species composition and absence (or very rare occurrence) of diagnostic species of the previous three sub-communities as well as constant presence of Stellaria nemorum (here and there as subdominant species, with cover above 5 or even 25\%). According to records available to the present, occurrence of Petasites albus is bound to this sub-community, however, only with low frequency and insignificant cover-abundance. This subassociation probably represents a transitional unit towards the association Mnio spinosi-Piceetum.

Floristically similar, yet more species-poor phytocoenoses could be developed on non-carbonate habitats: within this subassociation is classified one releve from glacigenic deposits close to the stream in the glacial valley of Dumbier Mt. (the western Low Tatras, Miadok (1995), p. 60, rel. 2: presence of Geranium sylvaticum, Primula elatior or Aconitum firmum, Delphinium oxysepalum, Petasites albus).

Nomenclatural note. - (A) The association name was originally described as an alternative name (see Samek et al., 1957, p. 15): "Piceetum (excelsae) altherbosum. Adenostylo-Piceetum". According to the current nomenclatural regulations (see Theurillat et al., 2021), such names are accepted as validly published names; the next available later syntaxonomical synonym is Adenostylo alliariaePiceetum excelsae Březina et Hadač in Hadač et al. 1969 nom. illeg. (Art. 31).

(B) Adenostylo-Piceetum Samek et al. 1957 is a younger homonym to the name Adenostylo-Piceetum Hartmann
1953 (cf. Art. 31). However, Hartmann abandoned his own name and as early as in the year 1959 when he introduced the name Athyrio alpestris-Piceetum Hartmann 1959 (validly published later as Athyrio alpestris-Piceetum Hartmann ex Hartmann et Jahn 1967).

Though, it remains unnoticed that the name Adenostylo-Piceetum Hartmann 1953 has a different syntaxonomical content in comparison to Athyrio alpestris-Piceetum Hartmann ex Hartmann et Jahn 1967: while the latter name is considered to represent a natural supramontane Picea abies woodland, the former name is based on published relevés of the unit "Luzulo nemorosae-Piceetum (Schmid et Gaisberg 1936) Br.-Bl. et Sissingh in Br.-Bl. et al. 1939, Luzula sylvatica facies of Bartch \& Bartsch (1940)" (cf. Hartmann, 1953, p. XIII of the Anhang) and later classified as Luzulo luzuloidis-Abietetum luzuletosum sylvaticae Oberdorfer 1957. Following the original description, this unit represents the montane and originally mixed Fagus-Abies woodlands of the class Carpino-Fagetea of the Black Forest, due to historical land management commonly with anthropogenically changed tree species composition in favour of conifers (especially Picea abies) and as such secondary forest community of the class Carpino-Fagetea (cf. Kučera, 2012a).

Therefore contrary to the traditional evaluations (cf. Exner 2007; Willner 2007, p. 240; Chytrý et al. 2013b and other authors), Adenostylo-Piceetum Hartmann 1953 and Athyrio alpestris-Piceetum Hartmann ex Hartmann et Jahn 1967 are not syntaxonomical synonyms and there is no real need to propose the latter name for conservation above Adenostylo-Piceetum Hartmann 1953 (cf. Willner (2007) vs. Kučera $\&$ Kliment (2011)).

(C) Willner (2007, p. 238) proposed the name Adenostylo alliariae-Piceetum Zukrigl 1973 nom. illeg. (Art. 31) for a nomenclatural conservation against the older unused homonym Adenostylo-Piceetum Hartmann 1953 and other three association with the syntaxonomically synonymical names used for Norway spruce forests of the Alps. However, this proposal was based on insufficient literature survey and, subsequently, ignored other older homonyms published for a natural calcareous supramontane Norway spruce woodland of the Western Carpathians (see Samek et al., 1957; Hadač et al., 1969 - published in German language) which have chronological priority above Zukrigl's (1973) name (cf. Kučera \& Kliment, 2011; Kučera, 2012a).

Instead, the name Adenostylo alliariae-Piceetum abietis Samek et al. 1957 nom. corr. is here proposed for a conserved name (nomen conservandum) to have nomenclatural priority over the unused older homonym and non-synonymous Adenostylo-Piceetum Hartmann 1953 established for secondary mixed Carpino-Fagetea commu- 
nity (see above) as well as over younger homonym Adenostylo alliariae-Piceetum Zukrigl 1973 nom. illeg.

\section{Mnio spinosi-Piceetum abietis Hadač et al. 1969 nom. corr.}

Nomenclatural type: Hadač et al. (1969), p. 269, rel. 134, lectotypus (Kučera, 2010a, p. 834).

Original name: Mnio spinosi-Piceetum as. nova (Hadač et al. 1969, p. 266), i.e. Mnio spinosi-Piceetum excelsae Hadač et al. 1969 nom. inept. (Rec. 10C, Art. 44)

Syntax. syn.: Oxalido-Piceetum excelsae Březina et Hadač in Hadač et al. 1969, Polysticho lonchitidis-Piceetum W. Matuszkiewicz ex J. Matuszkiewicz 1977

Incl.: Piceetum excelsae normale Szafer et al. 1927 nom. illeg. p. p. min. (Art. 13a), Sorbo-Piceetum calcicolum Šmarda et al. 1971 facies oxalidetosum p. p. maj.

Pseud: Piceetum normale sensu Szafer et al. 1923 non Beger 1922 p. p. min. (cf. also Principle II)

Nomen fictum (phantom name): Sorbo-Piceetum calcicolum Pawłowski 1956 apud Šmarda et al. 1971

Non: Piceetum excelsae normale Sillinger 1933 nom. inval. (Art. 3d), Piceetum excelsae normale calcicolum Sillinger 1933 nom. illeg. (Art. 34a), Piceetum excelsae normale silicicolum Sillinger 1933 nom. illeg. (Art. 34a), Piceetum abietis oxalidetosum acetosellae silicicolum Krajina 1933 nom. inval. (Art. 3e), Oxalido-Piceetum auct. bohem. non Březina et Hadač in Hadač et al. 1969

Set of the most important diagnostic species: negative species differentiation.

Relevé data: Hadač et al. (1969), p. 269, rels. 131, 139; Šmarda et al. (1971), tab. 18, rels. 8, 14; Kobzáková 1987 (msc.), tab. 8, rel. 7; Černušáková (1994), tab. 1, rels. 4, 5 and tab. 2, rels. 1-3, 15; Kanka (2008), tab. 14, rels. 6, 7; see fig. 3 .

Picea abies stands with admixture of Sorbus aucuparia (ssp. glabrata), on places with the high canopy cover without the latter species. Acer pseudoplatanus was originally admixed in the tree layer, Larix decidua was eventually present in regions with its autochthonous distribution, in the lower altitudes Abies alba. Among the more frequent shrub species belong only Ribes petraeum.

This community considerably differs from the previous communities with its usually low-forb field layer character and with low number of present species (somewhere even less than 15-20 species) which commonly reach only low cover values (up to 5\%). The number of constant species is also considerably lower. Frequent relatively low total cover of the field layer corresponds to the mentioned fea- tures (not seldom up to $50 \%$ at most, here and there only 1-3\%, cf. Hadač et al. (1969), 269, 274).

The constant components of the stands are only Oxalis acetosella, Soldanella spp. (more frequently $S$. carpatica was noted), Senecio nemorensis agg., Vaccinium myrtillus, Mycelis muralis, Myosotis sylvatica and Prenanthes purpurea. In some stands among the more frequent species belong Adenostyles alliariae (usually with very low coverabundance), Valeriana tripteris, Homogyne alpina, Galeobdolon luteum agg., or Moneses uniflora, Corallorhiza trifida, Primula elatior or Phyteuma spicatum, Ranunculus platanifolius; on some habitats also Polystichum lonchitis, Lycopodium annotinum, Vaccinium vitis-idaea, Viola biflora (cf. Adamczyk, 1962).

The field layer does not have a dominant species or, alternatively, Oxalis acetosella could reach cover over $50 \%$ in some places; eventually Adenostyles alliariae or Athyrium spp. also grow abundantly.

The ground layer does not have a specific constant species according to the currently known records. In some stands the dominant moss species are Dicranum scoparium and Plagiomnium cuspidatum, constantly accompanied by Hylocomium splendens and Brachythecium velutinum (Hadač et al., 1969, p. 274-275). Occurrence of Sphagnum girgensohnii was sporadically noted.

Variability. - With respect to the low total number of recorded phytocoenological relevés (i.e. with addition of originally unclassified relevés of Hadač et al. (1969), see Methods above), the three following subcommunities are differentiated:

(1) subassociation Mnio spinosi-Piceetum typicum subass. nov. hoc loco (nomenclatural type: Hadač et al. (1969), p. 269, rel. 134, holotypus hoc loco, automatical holotype sensu Art. 5b; differential species: Corallorhiza trifida, Hieracium murorum, Moneses uniflora, Primula elatior, Solidago virgaurea; original diagnosis: Hadač et al. (1969), p. 269, rels. 131, 134, 139 and p. 274, rels. 30, 32, 34, 55; Šmarda et al. (1971), tab. 18, rels. 8, 14; Kanka (2008), tab. 14, rels. 6, 7) which splits into two variants: (a) Valeriana tripteris-variant (incl. OxalidoPiceetum Hadač et al. 1969 s. str.) (differential species: Athyrium filix-femina, Cicerbita alpina, Homogyne alpina, Luzula sylvatica, Polygonatum verticillatum, Valeriana tripteris; Brachythecium velutinum, Dicranum scoparium, Plagiomnium cuspidatum) with characteristic distinctive dominace of Oxalis acetosella, and (b) Mycelis muralisvariant (三 Mnio spinosi-Piceetum Hadač et al. 1969 sensu strictissimo) (without differential species) which includes woodland stands with usually very the low cover of the field layer (1-3\%). 
(2) subassociation Mnio spinosi-Piceetum phyteumatetosum spicati P. Kučera subass. nov. hoc loco (nomenclatural type: Černušáková (1994), tab. 2, rel. 15, holotypus hoc loco; differential species: Athyrium distentifolium, Gentiana asclepiadea, Phyteuma spicatum, Ranunculus platanifolius, Veratrum album ssp. lobelianum; original diagnosis: Černušáková (1994), tab. 1, rel. 4 and tab. 2, rels. 1-3, 15) contains physiognomically different more or less tall-forb stands. Adenostyles alliariae-variant (differential species: Adenostyles alliariae, Athyrium distentifolium, Luzula sylvatica, Myosotis sylvatica, Phyteuma spicatum, Ranunculus platanifolius) is characterized by dominance of Adenostyles alliariae (cover up to 25-50\%). In the Ribes petraeum-variant (differential species: cf. Athyrium filix-femina, Ribes petraeum) species Athyrium filix-femina dominates and differential species of the former variant are absent.

(3) subassociation Mnio spinosi-Piceetum melampyretosum sylvatici $\mathrm{P}$. Kučera subass. prov. (nomenclatural type: Černušáková (1994), tab. 1, rel. 5, pro holotypus; differential species: Galium schultesii, Melampyrum sylvaticum; original diagnosis: Kobzáková (1987) (msc.), tab. 8, rel. 7; Černušáková (1994), tab. 1, rel. 5;) differs from the previous two sub-communities by absence of their differential species (Athyrium distentifolium could be present). However, this unit includes only two known relevés, including one relevé of Kobzáková (1987) from the non-carbonate region of the Západné Tatry Mts. (with presence of species Astrantia major, Galeobdolon lutem agg., Galium schultesii, Rubus saxatilis, Aruncus vulgaris, Ranunculus lanuginosus). ${ }^{12}$

Nomenclature. - Březina and Hadač (in Hadač et al. (1969)) validly published two new names of the syntaxonomically very close communities (cf. Kučera, 2012a): (1) Mnio spinosi-Piceetum proposed as a new association and (2) Oxalido-Piceetum originally proposed as nomen novum for the name Piceetum abietis oxalidetosum acetosellae silicicolum Krajina 1933. However, the latter name was invalidly published (see Kučera in red.) therefore the correct author citation for the respective Oxalido-Piceetum community of the Belianske Tatry Mts (Hadač et al., 1969) is Oxalido-Piceetum excelsae Březina et Hadač in Hadač et al. 1969.

These two communities are merged to one association due to their floristical and ecological similarity (see Kučera, 2012a). In respect of potential nomenclatural and also syntaxonomical problems with the name $O x a-$ lido-Piceetum (Kučera, in red.), the respective association

12 In respect of the relevé species composition, the original misidentification of the locality might be possible. is labelled with the unequivocal name Mnio spinosi-Piceetum Hadač et al. 1969.

\section{Hieracio murorum-Piceetum abietis \\ P. Kučera ass. nov. hoc loco}

Nomenclatural type: Kučera (2012a), p. 288, rel. 5, lectotypus hoc loco.

Set of the most important diagnostic species:

$\mathrm{E}_{1}$ : Agrostis capillaris, Calamagrostis villosa, Luzula luzuloides, *Stellaria nemorum, Deschampsia cespitosa, ${ }^{*}$ Calamagrostis arundinacea,

$\mathrm{E}_{0}$ : Polytrichum formosum, Pellia endiviifolia.

Relevé data and original diagnosis (see Table 3): Kučera (2002) (msc.), tab. 5, rels. 4-6; Kučera (2012a), p. 288, rel. 5 and p. 289, rels. 9-10; see fig. 2.

Canopy of the currently known stands is formed only by Picea abies. However, native tree species were originally also Acer pseudoplatanus, Sorbus aucuparia, in the lower altitudes Abies alba, and eventually sporadic Fagus sylvatica with low growth.

Field layer of the hitherto known phytocoenoses is dominated by Calamagrostis villosa. Very abundant are also Stellaria nemorum and Vaccinium myrtillus. Higher covers are here and there reached by Athyrium filix-femina, Luzula sylvatica ssp. sylvatica or Senecio hercynicus. Frequent components of the stands are Adenostyles alliariae, Calamagrostis arundinacea, Dryopteris dilatata, D. expansa.

Presence of calcicoles and nutrient-demanding species is reduced, however, this association unequivocally belong to the group of calcicolous natural Norway spruce communities which is justified by presence patterns of the species D. filix-mas, Hieracium murorum, Myosotis sylvatica, Stellaria nemorum and occasional occurrence of species as Alchemilla sp. div., Geranium sylvaticum, Primula elatior and so on (see Kučera in prep., tab. 2).

A characteristic feature of the hitherto known stands is the presence of species Agrostis capillaris, Alchemilla sp. div., Anthoxanthum odoratum, Deschampsia cespitosa, $H y$ pericum maculatum, Phleum rhaeticum, Potentilla aurea etc., which indicate impact of the historical land management (high mountain grazing and deforestation).

In contrast to the previous calcicolous communities, the ground layer is regularly developed with higher cover. In the ground layer Polytrichum formosum usually dominates, sporadically also $P$. commune, both species could reach cover above $5 \%$. Frequently are growing species Dicranum scoparium and Plagiothecium curvifolium, less frequently calcicole Pellia endiviifolia. 
Table 3: Original diagnosis of the association Hieracio murorum-Piceetum abietis P. Kučera 2022 ass. nov.

Tabela 3: Originalni opis asociacije Hieracio murorum-Piceetum abietis P. Kučera 2022 ass. nov.

\begin{tabular}{|c|c|c|c|c|c|c|c|c|c|c|c|c|c|}
\hline Rel. No. & 1 & 2 & 3 & 4 & 5 & 6 & Rel. No. & 1 & 2 & 3 & 4 & 5 & 6 \\
\hline Tree and shrub species & & & & & & & Dryopteris filix-mas & + & $\mathrm{r}$ & + & 2 & . & . \\
\hline $\mathrm{E}_{3}$ & & & & & & & Myosotis sylvatica & + & . & . & + & . & + \\
\hline Picea abies & 4 & 5 & 4 & 4 & 3 & 4 & Athyrium distentifolium & 1 & . & . & . & + & + \\
\hline $\mathrm{E}_{1}$ & & & & & & & Soldanella carpatica & + & + & + & . & . & . \\
\hline Picea abies & + & + & + & . & + & + & Avenella flexuosa & + & + & . & + & . & . \\
\hline Sorbus aucuparia & r & $\mathrm{r}$ & + & + & . & + & Deschampsia cespitosa & + & . & . & 1 & . & + \\
\hline Acer pseudoplatanus & + & + & + & . & . & + & Rumex alpestris & + & . & . & $\mathrm{r}$ & . & + \\
\hline Fagus sylvatica & $\mathrm{r}$ & $\mathrm{r}$ & . & . & $\mathrm{r}$ & . & Alchemilla sp. div. & . & . & . & + & + & + \\
\hline Salix silesiaca & . & . & . & $\mathrm{r}$ & . & . & Ranunculus repens & 1 & . & . & + & . & . \\
\hline Field layer species & & & & & & & Anthoxanthum odoratum & + & + & . & . & . & . \\
\hline Calamagrostis villosa & 4 & 1 & 1 & 3 & 5 & 2 & Viola biflora & + & . & + & . & . & . \\
\hline Vaccinium myrtillus & 2 & 2 & 2 & 3 & 1 & 2 & Phegopteris connectilis & + & . & . & . & + & . \\
\hline Stellaria nemorum & 2 & 2 & 2 & 2 & 2 & 2 & Prenanthes purpurea & $\mathrm{r}$ & . & . & . & . & $\mathrm{r}$ \\
\hline Dryopteris carthusiana agg. & 2 & 1 & + & 2 & 2 & 2 & Poa annua & . & + & + & . & . & . \\
\hline Luzula sylvatica ssp. sylvatica & 2 & + & + & 1 & 1 & 1 & Veronica chamaedrys & . & . & . & $\mathrm{r}$ & . & + \\
\hline Oxalis acetosella & 1 & + & 1 & + & + & + & Campanula serrata & . & . & . & . & + & + \\
\hline Luzula luzuloides & 1 & + & + & 1 & 1 & 2 & Hypericum maculatum & . & . & . & . & + & + \\
\hline Homogyne alpina & + & + & + & 1 & 1 & 2 & Ground layer species & & & & & & \\
\hline Rubus idaeus & + & $\mathrm{r}$ & + & 1 & + & + & Polytrichum formosum & 1 & 2 & 2 & 2 & . & 2 \\
\hline Senecio nemorensis agg. & 2 & 1 & + & - & 1 & + & Plagiothecium curvifolium & 1 & + & + & . & . & + \\
\hline Athyrium filix-femina & 2 & 1 & + & . & 2 & + & Dicranum scoparium & + & + & 1 & . & . & 1 \\
\hline Adenostyles alliariae & 1 & $\mathrm{r}$ & + & . & 1 & 1 & Pellia endiviifolia & . & 1 & + & . & . & . \\
\hline Calamagrostis arundinacea & + & + & . & 1 & 1 & 2 & Pellia sp. & . & . & . & . & . & + \\
\hline Agrostis capillaris & + & + & . & + & + & + & Polytrichum commune & 2 & 2 & . & . & . & . \\
\hline
\end{tabular}

\section{Field and ground layer species present in one relevé only:}

Rel. 1: $\mathrm{E}_{1}$ : Epilobium montanum +, Galium odoratum +, Primula elatior +; $-\mathrm{E}_{0}$ : Brachythecium starkei + .

Rel. 2: $\mathrm{E}_{1}$ : Gnaphalium sp. r, Potentilla aurea $\mathrm{r} ;-\mathrm{E}_{0}$ : Atrichum undulatum 2.

Rel. 3: $\mathrm{E}_{1}$ : Chrysosplenium alternifolium + , Maianthemum bifolium + , Phleum rhaeticum + , Allium victorialis $\mathrm{r}$, Veratrum album ssp. lobelianum $\mathrm{r} ;-\mathrm{E}_{0}$ : Calypogeia azurea + , Dicranella heteromalla.+

Rel. 4: $\mathrm{E}_{1}$ : Tussilago farfara 1, Galeopsis speciosa + , Nardus stricta + , Urtica dioica + .

Rel. 5: $\mathrm{E}_{1}$ : Senecio subalpinus +.

Rel. 6: $\mathrm{E}_{1}$ : Festuca sp. 1, Chaerophyllum hirsutum +, Geranium sylvaticum +, Phleum pratense +, Polygonatum verticillatum +; $-\mathrm{E}_{0}$ : Plagiomnium rostratum + .

\section{Localities:}

Rel. 1: Kučera 2012a, p. 289, rel. 9 - Vel'ká Fatra Mts, Borišov Mt., right part of the Prvý Balov, near the ridge, 1407 m a.s.l., 19.8.2008, P. Kučera (PK173).

Rel. 2: Kučera 2012a, p. 289, rel. 10 - Vel'ká Fatra Mts, Borišov Mt., right part of the Prvý Balov, next to the forest line near a dell/avalanche track, 1427 m a.s.l., 26.7.2006, P. Kučera (PK113).

Rel. 3: Kučera 2012a, p. 288, rel. 5 - Vel'ká Fatra Mts, Borišov Mt., on the ridge between the Prvý Balov and Druhý Balov, 1447 m a.s.1., 23.7.2003, P. Kučera (PK63).

Rel. 4: Kučera 2002 (msc.), tab. 5, rel. 5 - Vel'ká Fatra Mts, Borišov Mt., right part of the Prvý Balov, ca. 1400 m a.s.l., 25.8.2001, P. Kučera (PK48); originally classified as "Athyrio alpestris-Piceetum Hartmann 1959".

Rel. 5: Kučera 2002 (msc.), tab. 5, rel. 6 - Vel'ká Fatra Mts, Borišov Mt., right part of the Prvý Balov, ca. 1405 m a.s.l., 25. 8.2001, P. Kučera (PK49); originally classified as “Athyrio alpestris-Piceetum Hartmann 1959".

Rel. 6: Kučera 2002 (msc.), tab. 5, rel. 4 - Vel'ká Fatra Mts, Borišov Mt., right part of the Prvý Balov, ca. 1450 m a.s.l., 25.8.2001, P. Kučera (PK45); originally classified as "Athyrio alpestris-Piceetum Hartmann 1959". 
To the present, phytocoenoses of the association Hieracio murorum-Piceetum were recorded only in the Vel'ká Fatra Mts., in the northern slopes of Borišov Mt., where they are protected within the Borišov National Nature Reserve. Originally the respective woodland stands were widely distributed on the whole main ridge of the Vel'ká Fatra Mts, and most probably also elsewhere within the Western Carpathians, e.g. the Malá Fatra Mts. (Stoh Mt.), the eastern Low Tatras (Vel'ký Bok Mt.).

In all the mentioned areas, the geological background is formed by less resistant calcareous rocks of the Mráznica formation, i.e. grey marly limestones, marlstones and marly shales (Polák et al., 1997) and, consequently, the relief of the slopes is smooth-faced, without rugged ridges, rocky ribs and rock cuts and only exceptionally with bouldery taluses. This terrain characteristic was used for extensive (pre-)historical deforestation, altitudinally from the ridges deeply to the horizon of the mixed montane Fagus woodland, therefore the original supramontane Norway spruce stands are usually not preserved. The recorded old stands of the Borišov Mt. are also influenced by historical (partly present) mountain grazing (see above).

In places where the natural habitat development would conclude with blocking the influence of the carbonate background, phytocoenoses of the alliance Piceion abietis Pawłowski ex Pawłowski et al. 1928 nom. corr. could develop and replace the Hieracio murorum-Piceetum woodland. Such stands did not preserve due to the mentioned large-scale deforestation.

\section{Higher syntaxonomical units of calcareous Norway spruce woodlands}

The above-presented Western Carpathian and other similar European syntaxa of Picea abies communities are traditionally classified within the order Athyrio-Piceetalia Hadač 1962 or, recently, under the order name "Athyrio filicis-feminae-Piceetalia Hadač ex Hadač et al. 1969" (Kučera, 2010a; Mucina et al., 2016 and other following authors). The latter name was proposed to be the first validly published counterpart of the supposedly invalid name Athyrio-Piceetalia Hadač 1962. However, the corresponding nomenclatural construction was incorrect because two of the totally three subordinated alliances were in fact validly published by Hadač (1962) (cf. Kučera, in red.).

Thorough revision of the original diagnoses of the alliances Oxalido-Piceion (Krajina 1933) Březina et Hadač in Hadač 1962 and Chrysanthemo-Piceion (Krajina 1933) Březina et Hadač in Hadač 1962, i.e. Oxalidion acetosellae Krajina 1933 and Chrysanthemion rotundifolii Krajina
1933, demonstrated that later application of the respective alliances is syntaxonomically incompatible with their original phytocoenotical delimitation. The factual utilization of these alliance names as pseudonyma, i.e. "Oxalido-Piceion auct. non (Krajina 1933) Březina et Hadač in Hadač 1962" and "Chrysanthemo-Piceion auct. non (Krajina 1933) Březina et Hadač in Hadač 1962" has begun in the vegetation surveys of Holub et al. (1967) and Hadač et al. (1969) and continues to the present (Kučera, in red.).

As the syntaxonomical delimitation of the order Athyrio-Piceetalia Hadač 1962 is defined by its original diagnosis, i.e. alliances Oxalido-Piceion (Krajina 1933) Březina et Hadač in Hadač 1962 and Chrysanthemo-Piceion (Krajina 1933) Březina et Hadač in Hadač 1962, the common application of this order name equally corresponds to the pseudonym Athyrio-Piceetalia auct. non Hadač 1962 (Kučera, in red.). The pseudonymical approach to the name was already expressed in Hadač's (1962, p. 53) own short ecological and floristical characteristics of the order and constantly continues to the present (cf. Mucina et al., 2016; Dubyna et al., 2019; Bergmeier, 2020). The here presented name Cortuso-Piceetalia is published to replace the pseudonym Athyrio-Piceetalia auct. non Hadač 1962.

\section{Cortuso matthioli-Piceetalia abietis P. Kučera ord. nov. hoc loco}

Nomenclatural type: alliance Cortuso matthioli-Piceion abietis P. Kučera 2022 (see below p. 141), holotypus hoc loco

Pseudonym: Athyrio-Piceetalia auct. non Hadač 1962

Original diagnosis: Cortuso matthioli-Piceion abietis P. Kučera 2022 [see below p. 141], Melico nutantis-Piceion (Kielland-Lund 1981) P. Kučera 2022 [see below p. 139], Calamagrostio variae-Pinion cembrae P. Kučera 2017 [Kučera, 2017, p. 414], Seslerio caeruleae-Pinion uncinatae Vigo 1974 [Vigo, 1974, p. 53]

Differential species (see Table 4): ${ }^{13}$

$\mathrm{E}_{2}$ : (Ribes petraeum);

$\mathrm{E}_{1}$ : Daphne mezereum, Ribes petraeum, Acer pseudoplatanus, (Lonicera nigra);

Valeriana tripteris, Primula elatior, Phyteuma spicatum, Cortusa matthioli, Polygonatum verticillatum, Hieracium murorum, Viola biflora, Myosotis sylvatica, Geranium sylvaticum, Calamagrostis varia, Asplenium viride, Astrantia major, Cirsium erisithales, Cicerbita alpina, Leucanthemum rotundifolium, Galeobdolon luteum agg., Heracleum sphondylium, Polystichum lonchitis, Crepispaludosa, Mycelis

13 Species with the fidelity value $(\varphi \times 100)$ lower that " 33 " are in brackets. 
Table 4: Differential table of the supramontane Norway spruce woodlands of the class Vacccinio-Piceetea Br.-Bl. in Br.-Bl. et al. 1939 in Slovakia with values of constancy $(\%)$ and fidelity $(\varphi \times 100)$ in the exponent.

Table 4: Diferencialna tabela supramontanskih smrekovih gozdov razreda Vacccinio-Piceetea Br.-Bl. in Br.-Bl. et al. 1939 na Slovaškem s prikazano stalnostjo $(\%)$ in nadpisano navezanostjo $(\varphi \times 100)$.

The relevé dataset is identical with the Tabble 2 (synoptic table of the order Cortuso matthioli-Piceetalia) and the Table 3 (synoptic table of the order Piceetalia abietis) compiled by Kučera (in prep.).

Species with fidelity value $(\varphi \times 100)$ lower than 25 are omitted.

Group 1 - Cortuso matthioli-Piceetalia abietis P. Kučera 2022 ord. nov.

Group 2 - Piceetalia abietis Pawłowski ex Pawłowski et al. 1928 nom. corr.

\begin{tabular}{|c|c|c|c|c|c|}
\hline Group No. & 1 & 2 & Group No. & 1 & 2 \\
\hline No. of relevés & 95 & 185 & Sesleria caerulea & $25^{38.0}$ &.$^{-}$ \\
\hline Tree and shrub species & & & Thalictrum aquilegiifolium & $22^{35.3}$ &.$^{-}$ \\
\hline $\mathbf{E}_{2}$ & & & Pyrethrum clusii & $22^{35.3}$ &.$^{-}$ \\
\hline Ribes petraeum & $16^{26.5}$ & $1^{-}$ & Senecio subalpinus & $22^{35.3}$ &.$^{-}$ \\
\hline $\mathbf{E}_{\mathbf{1}}$ & & & Maianthemum bifolium & $26^{34.6}$ & $2^{-}$ \\
\hline Daphne mezereum & $47^{55.7}$ &.$^{-}$ & Senecio nemorensis agg. & $72^{34.4}$ & $37^{-}$ \\
\hline Ribes petraeum & $31^{36.4}$ & $3^{-}$ & Chrysosplenium alternifolium & $20^{33.3}$ &. \\
\hline Acer pseudoplatanus & $20^{33.3}$ &.$^{-}$ & Cystopteris fragilis & $20^{33.3}$ &.$^{-}$ \\
\hline Lonicera nigra & $37^{31.3}$ & $10^{-}$ & Campanula rotundifolia agg. & $22^{31.7}$ & $2^{-}$ \\
\hline$\overline{\text { Field layer species }\left(\mathrm{E}_{1}\right)}$ & & & Cystopteris montana & $18^{31.3}$ &.$^{-}$ \\
\hline Valeriana tripteris & $74^{73.7}$ & $2^{-}$ & Pimpinella major & $18^{31.3}$ &.$^{-}$ \\
\hline Primula elatior & $56^{62.2}$ &.$^{-}$ & Adenostyles alliariae & $72^{31.3}$ & $41^{-}$ \\
\hline Phyteuma spicatum & $55^{60.6}$ & $1^{-}$ & Lilium martagon & $19^{31.0}$ & $1^{-}$ \\
\hline Cortusa matthioli & $52^{59.0}$ &.$^{-}$ & Alchemilla sp. div. & $19^{31.0}$ & $1^{-}$ \\
\hline Polygonatum verticillatum & $60^{57.5}$ & $6^{-}$ & Moneses uniflora & $20^{30.8}$ & $1^{-}$ \\
\hline Hieracium murorum & $61^{56.3}$ & $8^{-}$ & Ranunculus lanuginosus & $17^{30.3}$ &.$^{-}$ \\
\hline Viola biflora & $47^{55.7}$ &.$^{-}$ & Geum rivale & $17^{30.3}$ &.$^{-}$ \\
\hline Myosotis sylvatica & $47^{55.7}$ &.$^{-}$ & Aconitum variegatum & $17^{30.3}$ &.$^{-}$ \\
\hline Geranium sylvaticum & $43^{51.6}$ & $1^{-}$ & Dentaria enneaphyllos & $17^{30.3}$ &.$^{-}$ \\
\hline Calamagrostis varia & 4150.8 &.$^{-}$ & Rubus saxatilis & $18^{30.0}$ & $1^{-}$ \\
\hline Asplenium viride & $41^{50.8}$ &.$^{-}$ & Veratrum allbum ssp. lobelianum & $46^{29.9}$ & $18^{-}$ \\
\hline Astrantia major & $39^{49.2}$ &.$^{-}$ & Prenanthes purpurea & $57^{28.5}$ & $29^{-}$ \\
\hline Cirsium erisithales & $39^{49.2}$ &.$^{-}$ & Carex digitata & $15^{28.2}$ &.$^{-}$ \\
\hline Cicerbita alpina & $38^{48.3}$ &.$^{-}$ & Carduus glaucinus & $14^{27.1}$ &.$^{-}$ \\
\hline Leucanthemum rotundifolium & $39^{48.2}$ & $1^{-}$ & Phyteuma orbiculare & $14^{27.1}$ &.$^{-}$ \\
\hline Galeobdolon luteum agg. & $40^{47.3}$ & $2^{-}$ & Ranunculus breyninus & $13^{26.0}$ &.$^{-}$ \\
\hline Heracleum sphondylium & $36^{46.7}$ &.$^{-}$ & Crepis jacquinii & $13^{26.0}$ &.$^{-}$ \\
\hline Polystichum lonchitis & $36^{46.7}$ &.$^{-}$ & Sesleria tatrae & $13^{26.0}$ &.$^{-}$ \\
\hline Crepis paludosa & $35^{45.8}$ &.$^{-}$ & Carex sempervirens ssp. tatrorum & $13^{26.0}$ &.$^{-}$ \\
\hline Mycelis muralis & $36^{44.8}$ & $1^{-}$ & Calamagrostis villosa & $25^{-}$ & $75^{49.9}$ \\
\hline Fragaria vesca & $32^{43.3}$ &.$^{-}$ & Avenella flexuosa & $51^{-}$ & $89^{42.1}$ \\
\hline Clematis alpina & $31^{42.4}$ &.$^{-}$ & Dryopteris carthusiana agg. & $54^{-}$ & $90^{40.0}$ \\
\hline Galium schultesii & $32^{42.3}$ & $1^{-}$ & Vaccinium myrtillus & $77^{-}$ & $99^{33.8}$ \\
\hline Dryopteris filix-mas & $38^{42.0}$ & $4^{-}$ & Lycopodium annotinum & $3^{-}$ & $19^{25.7}$ \\
\hline Ranunculus platanifolius & $37^{41.1}$ & $4^{-}$ & Ground layer species $\left(\mathrm{E}_{\mathbf{0}}\right)$ & & \\
\hline Soldanella marmarossiensis agg. & $49^{40.7}$ & $12^{-}$ & Mnium spinosum & $38^{47.4}$ & $1^{-}$ \\
\hline Chaerophyllum hirsutum & $32^{40.3}$ & $2^{-}$ & Ctenidium molluscum & $23^{36.2}$ &.$^{-}$ \\
\hline Epilobium montanum & $32^{39.3}$ & $2^{-}$ & Tortella tortuosa & $24^{32.6}$ & $2^{-}$ \\
\hline Bellidiastrum michelii & $26^{38.9}$ &.$^{-}$ & Polytrichum formosum & $19^{-}$ & $71^{52.7}$ \\
\hline Mercurialis perennis & $26^{38.9}$ &.$^{-}$ & Calypogeia integristipula &.$^{-}$ & $23^{36.3}$ \\
\hline Soldanella carpatica & $28^{38.5}$ & $1^{-}$ & Dicranum scoparium & $53^{-}$ & $\mathbf{8 6}^{36.1}$ \\
\hline Cardaminopsis arenosa agg. & $25^{38.0}$ &.$^{-}$ & Sphagnum girgensohnii & $4^{-}$ & $22^{26.5}$ \\
\hline
\end{tabular}


muralis, Fragaria vesca, Clematis alpina, Galium schultesii, Dryopteris filix-mas, Ranunculus platanifolius, Soldanella marmarossiensis agg., Chaerophyllum hirsutum, Epilobium montanum, Bellidiastrum michelii, Mercurialis perennis, Soldanella carpatica, Cardaminopsis arenosa agg., Sesleria albicans, Thalictrum aquilegiifolium, Pyrethrum clusii, Senecio subalpinus, Maianthemum bifolium, Senecio nemorensis agg., Chrysosplenium alternifolium, Cystopteris fragilis, (Campanula rotundifolia agg., Cystopteris montana, Pimpinella major, Adenostyles alliariae, Lilium martagon, Alchemilla sp. div., Moneses uniflora, Ranunculus lanuginosus, Geum rivale, Aconitum variegatum, Dentaria enneaphyllos, Rubus saxatilis, Veratrum allbum ssp. lobelianum, Prenanthes purpurea, Carex digitata, Carduus glaucinus, Phyteuma orbiculare, Ranunculus breyninus, Crepis jacquinii, Sesleria tatrae, Carex sempervirens ssp. tatrorum);

$\mathrm{E}_{0}$ : Mnium spinosum, Ctenidium molluscum, (Tortella tortuosa).

Floristical delimitation. - The characteristic feature of communities of the order Cortuso matthioli-Piceetalia within the class Vaccinio-Piceetea is the presence of species which are - within the altitudinal vegetation zone of natural Picea abies woodland and natural (mixed) Pinus cembra woodland, i.e. in the supramontane vegetation zone - (almost exclusively) bounded to calcareous soils: Astrantia major, Chrysosplenium alternifolium, Crepis paludosa, Dentaria enneaphyllos, Fragaria vesca, Galium schultesii, Geranium sylvaticum, Heracleum sphondylium, Mercurialis perennis, Moneses uniflora, Mycelis muralis, Myosotis sylvatica, Phyteuma spicatum, Polystichum lonchitis, Primula elatior, Valeriana tripteris, Viola biflora and others (see above). Only sporadically and on special habitats some species of this group could occur in the communities of the order Piceetalia abietis Pawłowski ex Pawłowski et al. 1928 (see Kučera, 2019a, tab. 1).

Within the class Vaccinio-Piceetea, the fundamental differential element of the Cortuso matthioli-Piceetalia communities of the high mountain elevations is the presence of calciphytes, for example Asplenium viride, Calamagrostis varia, Carduus glaucinus, Cirsium erisithales, Cortusa matthioli, Cystopteris montana, Phyteuma orbiculare, Pimpinella major or Sesleria albicans. These species not seldom constitute a significant share of the species composition of phytocoenoses or of their total cover (cf. Hadač et al., 1969; Fajmonová, 1978; Fajmonová, 1986; Kučera, 2012a). Reduced presence of calciphytes is limited to marginal communities of the order (e.g. Hieracio murorum-Piceetum P. Kučera 2022).

The ground layer of the Cortuso matthioli-Piceetalia communities is distinguished by presence of mosses Ctenidium molluscum, Mnium spinosum and Tortella tortuosa.
In contrast to generally accepted assessments that the class Vaccinio-Piceetea represents species-poor and acid woodland vegetation (e.g. Seibert, 1992; Chytrý in Chytrý et al., 2013), the respective calcareous natural Norway spruce woodlands constitute the proof that the Vaccinio-Piceetea communities comprise species-rich and very species-rich phytocoenoses as well. The concept of Vaccinio-Piceetea diagnostic taxa should also be revised: instead of species growing within large diversity of forest and non-forest vegetation (such as Vaccinium myrtillus, V. vitis-idaea), species common for both Piceetalia abietis and Cortuso-Piceetalia abietis syntaxa should be emphasized, i.e. (Central) European oreal species as Adenostyles alliariae, Homogyne alpina or Luzula sylvatica (cf. Kučera, in prep.).

Ecological delimitation. - Development and occurrence of the Cortuso matthioli-Piceetalia communities is dependent on trophically very favourable properties of soils which are neutral to slightly acidic (Šoltés, 1976), with intensive nitrification (Hadač et al., 1969). Soil pH could fall under 6 in deeper non-skeletal soils (Hadač et al., 1969). The soils are rendzinas (rendzic leptosols), pararendzinas (subgroup of rendzic leptosols) to cambisols (calcaric cambisols) which could be decalcified and acidified, however, deeper in the soil horizon they are at least neutral or slightly acidic (cf. Śály, 1986). The respective habitats are therefore found in mountain regions formed by carbonate rocks. In areas with extreme relief litosols (lithic leptosols) could be present.

Nutrient supply could be blocked by thicker humus layer developed on small areas within some communities: calcifuges are bound to such places (Vaccinium myrtillus). Only sporadically, on exceptionally favourable habitats, phytocoenoses of the order Cortuso matthioli-Piceetalia could develop on weathered non-carbonate rocks, generally on habitats influenced by increased moisture and nutrient supply.

Syntaxonomical delimitation. - Communities of the order Cortuso matthioli-Piceetalia are here divided to four basic subunits which are given the rank of an alliance.

(A) The alliance Cortuso matthioli-Piceion abietis P. Kučera 2022 (see below) comprises calcicolous Picea abies woodland types of the supramontane vegetation zone mainly of the Central European mountain ranges, with overlaps from the western (western Alps) to the southeastern part of the continent (Southern Carpathians). ${ }^{14}$ The question of the syntaxonomical classification of Norway

\footnotetext{
14 In the western half of the Alps Pinus uncinata Ramond ex DC s. str. could be admixed in more extreme habitats (cf. Ellenberg \& Klötzli, 1972; Schmider \& Burnand, 1988).
} 
spruce communities of the montane altitudes of the Dinaric mountain ranges (cf. Wraber, 1964; Wraber, 1969; Horvat et al., 1974; Zupančič, 1980; Accetto, 1993; Vukelić et al., 2010; Juvan et al., 2013 etc.) needs to be resolved in more detail: the refugial and relict occurrence of Picea abies (growing within mixed woodland phytocoenoses with Abies, Acer, Fagus etc., i.e. more or less marginal Carpino-Fagetea communities) should be carefully differentiated from the relict extragradal montane Cortuso matthioli-Piceetalia communities.

(B) Calcicolous Picea abies woodlands are replaced by calcicolous Pinus cembra or mixed Pinus cembra woodlands (mostly with Larix decidua) in the high mountain altitudes of the Alps as well as of the highest mountain ranges of the Carpathians (e.g. the Tatra Mountains). They are separated into the alliance Calamagrostio variae-Pinion cembrae P. Kučera 2017 based on phytocoenoses from the Belianske Tatry Mts (Western Carpathians). The respective woodland stands were significantly negatively influenced by historical land management and only their fragments were preserved or they are completely missing in large continuous areas (cf. Kanka, 2008; Zięba et al., 2018; Zięba et al., 2019; Kučera \& Barančok, 2021). Similar process could be presupposed also for large areas of the Alps outside of regions where the continuous Arolla pine woodlands were preserved up to the $20^{\text {th }}$ century.

(C) Geographically vicariant unit of the Pyrenees is the alliance Seslerio caeruleae-Pinion uncinatae Vigo 1974 (cf. Rivas-Martínez et al., 2001) with supramontane (to altimontane) plant communities formed by Pinus uncinata Ramond ex DC s. str. (and Abies alba). Data on the occurrence of phytocoenoses of this alliance in the northern Apennines (Angelini et al., 2009; Biondi et al., 2014) are questionable from the syntaxonomical as well as ecological point of view: from this region only the occurrence of Pinus mugo s. str. is indicated (cf. Gentile, 1995; Adorni, 2016; Pignatti et al., 2017).

(D) Occurrence of phytocoenoses floristically and ecologically belonging to the order Cortuso matthioliPiceetalia is also indicated from the northern Europe (cf. Kielland-Lund, 1981; Dierßen \& Dierßen, 1996). They syntaxonomically belong to the alliance Melico nutantis-Piceion (Kielland-Lund 1981) P. Kučera stat. nov. hoc loco (basionym: suballiance MelicoPiceenion Kielland-Lund 1981; see Kielland-Lund, 1981, p. $150,176,196) .{ }^{15}$

15 As Rivas-Martínez et al. (2011, p. 457) typified their name LinnaeoPiceion abietis (Br.-Bl. et Sissingh in Br.-Bl. et al. 1939) Rivas-Mart. in Rivas-Mart. et al. 2011 nom. illeg. (Art. 31) with the association name Piceetum fennnoscandicum Br.-Bl. in Br.-Bl. et al. 1939, that alliance name became a syntaxonomical synonym to calcicolous Melico nutantis-Piceion
However, similarly as in the Western Carpathians (see above p. 111-116), impacts of historical land management on the tree species composition of the Scandinavian woodlands should be carefully considered as Picea abies forests of the lower altitudes often represent secondary, substitutionary stands replacing potential natural mixed broadleaved forests of Quercus, Fagus, Acer spp.

(E) On the contrary, into the order Cortuso matthioli-Piceetalia (= Athyrio-Piceetalia auct. non Hadač 1962) do not belong various syntaxonomical units even if they are traditionally classified under the order name "Athyrio-Piceetalia Hadač 1962”. For example, Sýkora (1971) differentiated a new alliance Athyrio alpestris-Piceion Sýkora 1971 within the order Athyrio-Piceetalia Hadač 1962. It is not clear whether he used the original or the pseudonymical concept of the order name (cf. Kučera, in red.); however, if the only subordinated association Athyrio alpestris-Piceetum Hartmann ex Sýkora 1971 nom. illeg. (Art. 31, later synonym of Athyrio alpestris-Piceetum Hartmann ex Hartmann et Jahn 1967) is considered as a syntaxon of natural Picea abies woodland then it floristically belongs to the alliance Piceion abietis Pawłowski ex Pawłowski et al. 1928 nom. corr. (syntax. syn. Athyrio alpestris-Piceion Sýkora 1971) (cf. Šomšák, 1983; Exner, 2007; Kučera, 2010a; Kučera, 2010c; Kučera, 2012a; Chytrý et al., 2013b).

(F) As indicated above (p. 111-116), many Picea abies forest stands of the Western Carpathians are secondary, anthropogenic forest communities with the spontaneous secondary succession of Fagus sylvatica, and they should be syntaxonomically classified within the class Carpino-Fagetea. Besides the respective Picea forests, there were described some Abies alba syntaxa of the higher rank which are traditionally considered as Vaccinio-Piceetea syntaxa. Below are given some examples of units (or their common interpretation) which syntaxonomically do not belong into the order Cortuso matthioli-Piceetalia ( $=$ Athyrio-Piceetalia auct. non Hadač 1962).

(F1) Hadač (Hadač, 1962; Hadač, 1965, see also Hadač et al., 1969) differentiated an independent alliance Abietion albae within the order "Athyrio-Piceetalia Hadač 1962". This new syntaxon was proposed for Abies alba- and mixed Abies alba-Picea abies communities distributed in the southeastern part of the Belianske Tatry Mts (northern Slovakia) and the surrounding regions. Hadač considered them for natural coniferous woodlands with naturally absent Fagus sylvatica due to continental

(Kielland-Lund 1981) P. Kučera 2022: cf. the frequencies of species Geranium sylvaticum, Fragaria vesca, Melica nutans, Paris quadrifolia, Convallaria majalis within the original diagnosis of the association Piceetum fennnoscandicum Br.-Bl. in Br--Bl. et al. 1939 (Braun-Blanquet et al., 1939, p. 53-54; cf. also Kielland-Lund, 1981, tab. 39). 
climatic conditions. His concept was accepted in later Slovak regional or national surveys up to the present (for more detailed list see Kučera. in red.).

However, the factor which actually hindered the presence of $F$. sylvatica in the respective territory was historical land management, not presupposed continental climate. The supposed natural beechless character of the Abietion albae Hadač 1965 plant communities is of anthropogenic origin and they have to be classified within the class Carpino-Fagetea (Kučera, 2007; Kučera, 2010b; Kučera, 2012a; Kučera, 2012b and other works).

(F2) In the recent phytocoenological survey of the Austrian woodlands, Exner (2007) differentiated a special group of "dry carbonate Picea abies(-Abies alba) woodlands", classified as the suballiance Calamagrostio variaeAbietenion (Horvat 1962) Exner et Willner in Willner et al. 2007 within the class Vaccinio-Piceetea. However, the values of Fagus sylvatica frequency in the individual Austrian communities of this suballiance and, especially, increase in frequency of Fagus in the understorey in contrast to the canopy of the respective communities (see also Acer pseudoplatanus) as well as considerable decrease of frequency of Larix decidua (cf. Willner et al., 2007, tab. 33) indicate that spontaneous secondary succession takes place in the respective habitats and their tree species composition was substantially anthropogenically influenced.

Similarly like in the case of Abietion albae Hadač 1965 (see above), most of the respective secondary communities with anthropogenically lowered presence of $F$. sylvatica syntaxonomically belong to the class Carpino-Fagetea. Further research is needed to determine which Austrian regions exactly constitute the territory where $F$. sylvatica should be ultimately missing only due to natural reasons (e.g. a special intramountain climate). Corresponding Abies alba communities of the alliance Calamagrostio-Abietion Horvat 1962 nom. invers. in the Dinarides (Horvat, 1962; Horvat et al., 1974) should also be revised for historical anthropogenic influences and potential secondary succession of Fagus.

(F4) Similarly to the previous group, Austrian communities of Abieti-Piceenion Br.-Bl. in Br.-Bl. et al. 1939 (see Exner, 2007) represent for the substantial part anthropogenically influenced mixed forests with secondary succession of Fagus (cf. synoptic table No. 33 in Willner et al. (2007)) therefore the respective phytocoenoses should be classified within the class Carpino-Fagetea.

The nomenclatural type of the alliance Abieti-Piceion (Br.-Bl. in Br.-Bl. et al. 1939) Soó 1963/ Abieti-Piceenion Br.-Bl. in Br.-Bl. et al. 1939 - the association Piceetum montanum Br.-Bl. in Br.-Bl. et al. 1939 (Willner, 2007, p. 237) - also represents mixed coniferous forest with anthropogenically induced absence of $F$. sylvatica. The original diagnosis of this association consists of six published relevés of Beger (1922) from the Schanfigg Valley in the eastern Switzerland and from seven unpublished relevés of J. Braun-Blanquet. Although Beger (1922, p. 70) published only such phytocoenological relevés in which $F$. sylvatica is admixed, there exists also ca. pure F. sylvatica stand in the vicinity of Tschiertschen (coordinates $46^{\circ} 49^{\prime} 33^{\prime \prime} \mathrm{N}, 9^{\circ} 35^{\prime} 55^{\prime \prime} \mathrm{E}$, cca up to $1120 \mathrm{~m}$ a.s.l., cf. Google Earth). Fertile Fagus trees in this region were recorded even at $1330 \mathrm{~m}$ a.s.l. by Beger (1922).

Beger's relevés of Norway spruce communities from the lower montane altitudes represent secondary forests which developed under the millenia of human influence (the region is considerably deforested till the present) and as such they should be classified similarly as the Abietion albae Hadač 1965 communities of the northern Slovakia (Hadač, 1965; Hadač et al., 1969) within the class Carpino-Fagetea (cf. Hadač \& Sofron, 1980). Subsequently, the alliance should be classified with the respect to its original diagnosis in the same way as already Oberdorfer (1957) proposed. ${ }^{16}$

It seems that the spatial delimitation of the so-called "Zwischenalpen zone", i.e. the territory where F. sylvatica should be naturally absent (on the contrary to "Randalpen zone") and Picea abies and Abies alba form forests in the montane altitudes (Kuoch, 1954; Ellenberg, 1996; cf. Lauber et al., 2018) (see also Mayer \& Hofmann, 1969; Mayer, 1974; Mayer, 1984; Sauberer \& Willner, 2007), have to be substantially revised with serious consideration of the impacts of historical land management: the above mentioned Schanfigg Valley is commonly classified as the part of the Zwischenalpen zone, according Ellenberg \& Klötzli (1972) even within the Zentralalpen zone; however, the preserved Fagus stand by Tschiertschen and other Fagus occurrences towards the valley" opening contradict to such geobotanical classification (cf. Hess et al., 1967, p. 63).

In the Western Carpathians, an analogous Fagus-free "intramountain zone" was traditionally recognized; however, supposed natural absence of Fagus is, in fact, result of pre-/historical land management of the respective landscape, i.e. the intermountain basins of the Liptovská kotlina and the Popradská kotlina as well as the adjacent slopes of the surrounding mountain ranges (see Krippel, 1963; Kučera, 2012b).

(F5) Mucina et al. (2016) listed the alliance name Abietion albae Issler 1931 as a synonym of the alliance

16 In respect of the characteristics of the altitudinal distribution of the association Piceetum montanum Br.-Bl. in Br.-Bl. et al. 1939 and its original table by Braun-Blanquet et al. (1939, p. 15), the following typification is proposed for nomenclatural reasons: Piceetum montanum $\mathrm{Br}$.-Bl. in $\mathrm{Br}$.Bl. et al. 1939 [Braun-Blanquet et al. 1939, p. 14] Nomenclatural type: Beger 1922, p. 49-50, rel. No. 3, lectotypus hoc loco. 
Abieti-Piceion (Br.-Bl. in Br.-Bl. et al. 1939) Soó 1964 (cf. Willner, 2007, p. 237) which was presented as "Mesophilous fir forests on brown forest soils ...". However, the original diagnosis of the alliance Abietion albae Issler 1931 (Issler, 1926, tab. VI; Issler, 1931) clearly shows that the author classified in the respective units mixed AbiesFagus communities, probably also considerably influenced by historical land management (see differences of Fagus abundance between canopy and understorey). They syntaxonomically belong to the class Carpino-Fagetea (cf. Boeuf et al., 2014).

Slightly different is the case of the alliance Piceo--Abietion Ellenberg et Klötzli 1974 nom. inval. (Art. 3b), however, already the original authors classified their unit within the class Carpino-Fagetea (ut "Querco-Fagetea", Ellenberg \& Klötzli (1972), see also above "F4"). It should be also noted that Czech and Moravian phytocoenologists classify Abies alba forests within the class Carpino-Fagetea (Moravec et al., 2000; Boublík et al., 2013).

\section{Cortuso matthioli-Piceion abietis P. Kučera all. nov. hoc loco}

Nomenclatural type: Cirsio erisithalis-Piceetum abietis Fajmonová et P. Kučera in P. Kučera 2022 (see above p. 127), holotypus hoc loco.

Syntax. syn.: Piceion excelsae Sillinger 1933 p. p. min.

Pseudonyms (see Kučera in red.): Chrysanthemo-Piceion auct. non (Krajina 1933) Březina et Hadač in Hadač 1962, Oxalido-Piceion auct. non (Krajina 1933) Březina et Hadač in Hadač 1962

Original diagnosis (see the p. 119-136): Seslerio caeruleae--Piceetum abietis Fajmonová 1978 nom. corr. et nom. cons. propos., Cirsio erisithalis-Piceetum abietis Fajmonová et P. Kučera in P. Kučera 2022, Fragario vescae-Piceetum abietis P. Kučera 2022 ass. prov., Adenostylo alliariae-Piceetum abietis Samek et al. 1957 nom. corr. et nom. cons. propos., Mnio spinosi-Piceetum abietis Hadač et al. 1969 nom. corr. (incl. Oxalido-Piceetum Březina et Hadač in Hadač et al. 1969), Hieracio murorum-Piceetum abietis P. Kučera 2022

Differential species (see Table 5): ${ }^{17}$

$\mathrm{E}_{1}$ : Myosotis sylvatica, Primula elatior, Fragaria vesca, Senecio nemorensis agg., Galeobdolon luteum agg., Astrantia major, Mercurialis perennis, Dryopteris filix-mas, Sesleria albicans, Heracleum sphondylium, Pyrethrum clusii, Chaerophyllum hirsutum, Epilobium montanum, Lilium martagon, Milium effusum, Paris quadrifolia, Rubus saxa-

17 Species with the fidelity value lower that " 33 " are in brackets. tilis, Pimpinella major, Cirsium erisithales, Ranunculus lanuginosus, Dentaria enneaphyllos, (Soldanella carpatica, Cicerbita alpina, Mycelis muralis, Crepis paludosa);

$\mathrm{E}_{0}$ : (Tortella tortuosa, Ctenidium molluscum).

Floristical delimitation and characteristics. - Phytocoenoses of the order Cortuso matthioli-Piceetalia in the Western Carpathians are divided into two altitudinally and phytochorologically different geobotanical groups with diverse postglacial and pre-/historical development. While the Arolla pine woodlands of the alliance Calamagrostio variae-Pinion cembrae survived only in the most highest mountain ranges and even there its stands were preserved only in small isolated islands (cf. Kanka, 2008; Zięba et al., 2018; vs. Kučera, 2019b; Kučera \& Barančokc, 2021) due to extensive deforestation for high mountain grazing, the Cortuso-Piceion woodlands participate on formation of the distinct altitudinal vegetation zone developed in all higher mountain ranges with adequate total elevations (however, large continuous areas were deforested too).

The most noticeable differential feature of the Cortuso matthioli-Piceion phytocoenoses is the natural absence of mature Pinus cembra trees. Other species bound to high mountain altitudes are also (mostly) missing in Cortuso matthioli-Piceion: Rhodiola rosea, Festuca versicolor, Hedysarum hedysaroides, Salix retusa etc. For the list of positive differential species see the Table 5. Lower constancies of Vaccinium vitis-idaea, Huperzia selago, Avenella flexuosa in Cortuso matthioli-Piceion stands are caused by generally less extreme habitats and thinner accumulations of raw humus as well as probably only very sporadic development of tangel humus; however, anthropogenic absence of Pinus cembra woodlands on ecologically medium habitats with deeper calcareous soils causes certain species bias in the presented floristical comparison of the Table 5.

The canopy dominant of the Cortuso matthioli-Piceion woodlands is Picea abies to which other species are admixed, most frequently Sorbus aucuparia (usually ssp. glabrata). Not seldom Acer pseudoplatanus grows in the stands, in the ecologically more extreme habitats Sorbus aria is present. Natural presence of Abies alba is presupposed in the lower elevations of the altitudinal distribution of the Cortuso matthioli-Piceion woodlands, with occurrence of Fagus sylvatica is counted only at lower altitudinal limit of Norway spruce altitudinal vegetation zone and only with specimens of low growth which do not have ecological impact on development and species diversity of plant communities. In the region of the Tatra Mountains and the Low Tatras autochthonous Larix decidua was originally perhaps more represented in the stands, especially in more extreme habitats (in the similar way prob- 
Table 5: Differential table of the alliances of the order Cortuso matthioli-Piceetalia P. Kučera 2022 ord. nov. in Slovakia with values of constancy $(\%)$ and fidelity $(\varphi \times 100)$ in the exponent.

Tabela 5: Diferencialna tabela zvez redu Cortuso matthioli-Piceetalia P. Kučera 2022 ord. nov. na Slovaškem s prikazano stalnostjo

$(\%)$ in nadpisano navezanostjo $(\varphi \times 100)$.

The relevé dataset is identical with the Table 2 (synoptic table of the order Cortuso matthioli-Piceetalia) compiled by Kučera (in prep.). Species with fidelity value $(\varphi \times 100)$ lower than 25 are omitted.

Group 1 - Cortuso matthioli-Piceion abietis P. Kučera 2022 ord. nov., Group 2 - Calamagrostio variae-Pinion cembrae P. Kučera 2017

\begin{tabular}{|c|c|c|c|c|c|}
\hline Group No. & 1 & 2 & Group No. & 1 & 2 \\
\hline No. of relevés & 80 & 15 & Soldanella carpatica & $32^{32.5}$ & $7^{-}$ \\
\hline Tree and shrub species & & & Cicerbita alpina & $42^{32.5}$ & $13^{-}$ \\
\hline $\mathbf{E}_{3}$ & & & Mycelis muralis & $40^{30.2}$ & $13^{-}$ \\
\hline Pinus cembra &.$^{-}$ & $100^{100.0}$ & Crepis paludosa & $39^{29.0}$ & $13^{-}$ \\
\hline Betula carpatica &.$^{-}$ & $40^{50.0}$ & Vaccinium vitis-idaea & $22^{-}$ & $93^{71.7}$ \\
\hline Sorbus aucuparia & $35^{-}$ & $67^{31.7}$ & Campanula rotundifolia agg. & $12^{-}$ & $73^{61.5}$ \\
\hline $\mathrm{E}_{2}$ & & & Cystopteris montana & $9^{-}$ & $67^{59.8}$ \\
\hline Picea abies & $20^{-}$ & $67^{47.1}$ & Cystopteris fragilis & $12^{-}$ & $\mathbf{6 0}^{49.4}$ \\
\hline Sorbus aucuparia & $29^{-}$ & $73^{44.6}$ & Huperzia selago & $10^{-}$ & $\mathbf{5 3} 3^{46.6}$ \\
\hline Pinus cembra & $\cdot-$ & $27^{39.2}$ & Sesleria tatrae & $6^{-}$ & $\mathbf{4} 7^{45.8}$ \\
\hline Betula carpatica & $1^{-}$ & $20^{30.4}$ & Avenella flexuosa & $44^{-}$ & $87^{45.1}$ \\
\hline Pinus mugo & $14^{-}$ & $40^{29.6}$ & Bistorta major & $2^{-}$ & $33^{40.2}$ \\
\hline Lonicera nigra & $4^{-}$ & $20^{25.1}$ & Rhodiola rosea & $2^{-}$ & $33^{40.2}$ \\
\hline $\mathrm{E}_{1}$ & & & Gymnocarpium dryopteris & $16^{-}$ & $\mathbf{5 3}^{38.9}$ \\
\hline Pinus cembra & $1^{-}$ & $47^{53.2}$ & Festuca versicolor &.$^{-}$ & $20^{33.3}$ \\
\hline Sorbus aucuparia & $64^{-}$ & $100^{47.1}$ & Hedysarum hedysaroides &.$^{-}$ & $20^{33.3}$ \\
\hline Betula carpatica &.$^{-}$ & $27^{39.2}$ & Dryopteris carthusiana agg. & $49^{-}$ & $\mathbf{8 0}^{32.6}$ \\
\hline Picea abies & $32^{-}$ & $\mathbf{6 0}^{27.6}$ & Calamagrostis varia & $36^{-}$ & $67^{30.4}$ \\
\hline Field layer species $\left(\mathrm{E}_{1}\right)$ & & & Pyrola rotundifolia & $1^{-}$ & $20^{30.4}$ \\
\hline Myosotis sylvatica & $56^{62.6}$ &.$^{-}$ & Myosotis alpestris & $1^{-}$ & $20^{30.4}$ \\
\hline Primula elatior & $64^{51.8}$ & $13^{-}$ & Luzula luzuloides & $24^{-}$ & $\mathbf{5 3}^{30.4}$ \\
\hline Fragaria vesca & $38^{48.0}$ &.$^{-}$ & Salix retusa &.$^{-}$ & $13^{26.7}$ \\
\hline Senecio nemorensis agg. & $79^{45.8}$ & $33^{-}$ & Salix reticulata &.$^{-}$ & $13^{26.7}$ \\
\hline Galeobdolon luteum agg. & $46^{44.9}$ & $7^{-}$ & Bistorta vivipara &.$^{-}$ & $13^{26.7}$ \\
\hline Astrantia major & $45^{43.8}$ & $7^{-}$ & Astragalus norvegicus &.$^{-}$ & $13^{26.7}$ \\
\hline Mercurialis perennis & $31^{43.0}$ &.$^{-}$ & Ranunculus alpestris &.$^{-}$ & $13^{26.7}$ \\
\hline Dryopteris filix-mas & $44^{42.7}$ & $7^{-}$ & Saxifraga wablenbergii &.$^{-}$ & $13^{26.7}$ \\
\hline Sesleria caerulea & $30^{42.0}$ &.$^{-}$ & Helianthemum grandiflorum &.$^{-}$ & $13^{26.7}$ \\
\hline Heracleum sphondylium & $41^{40.5}$ & $7^{-}$ & Androsace chamaejasme &.$^{-}$ & $13^{26.7}$ \\
\hline Pyrethrum clusii & $26^{38.9}$ &.$^{-}$ & Gentiana punctata &.$^{-}$ & $13^{26.7}$ \\
\hline Chaerophyllum hirsutum & $36^{36.0}$ & $7^{-}$ & Bartsia alpina &.$^{-}$ & $13^{26.7}$ \\
\hline Epilobium montanum & $36^{36.0}$ & $7^{-}$ & Dryas octopetala &.$^{-}$ & $13^{26.7}$ \\
\hline Lilium martagon & $22^{35.6}$ &.$^{-}$ & Saxifraga paniculata &.$^{-}$ & $13^{26.7}$ \\
\hline Milium effusum & $21^{34.5}$ &.$^{-}$ & Moehringia muscosa &.$^{-}$ & $13^{26.7}$ \\
\hline Paris quadrifolia & $21^{34.5}$ & $\cdot^{-}$ & Ground layer species $\left(\mathrm{E}_{\mathbf{0}}\right)$ & & \\
\hline Rubus saxatilis & $21^{34.5}$ &.$^{-}$ & Tortella tortuosa & $29^{41.0}$ &.$^{-}$ \\
\hline Pimpinella major & $21^{34.5}$ &.$^{-}$ & Ctenidium molluscum & $28^{39.9}$ &.$^{-}$ \\
\hline Cirsium erisithales & $44^{33.7}$ & $13^{-}$ & Plagiothecium undulatum &.$^{-}$ & $13^{26.7}$ \\
\hline Ranunculus lanuginosus & $20^{33.3}$ &.$^{-}$ & Mylia taylorii &.$^{-}$ & $13^{26.7}$ \\
\hline Dentaria enneaphyllos & $20^{33.3}$ &.$^{-}$ & Ptilidium ciliare &.$^{-}$ & $13^{26.7}$ \\
\hline
\end{tabular}


ably also Betula carpatica). The current Larix abundance is increased (or on the contrary decreased) by silvicultural interventions and by historical land management.

Regular components of the understorey are in particular species Daphne mezereum, Lonicera nigra, Ribes petraeum and $R$. alpinum. Pinus mugo naturally occurs on more rocky habitats.

Characteristic feature of the prevalent part of communities of the alliance Cortuso matthioli-Piceion is the high species diversity (sporadically over 80 species in one relevé). Important part of the field layer of phytocoenoses is presence of calciphytes, for example Cirsium erisithales, Asplenium viride, Bellidiastrum michelii, Cardaminopsis arenosa agg., Rubus saxatilis, Pimpinella major, in some cases they are dominant components of stands Calamagrostis varia, Cortusa matthioli, Sesleria albicans.

Distinctive diagnostic feature of the alliance Cortuso matthioli-Piceion against the alliance Piceion abietis Pawłowski ex Pawłowski et al. 1928 (order Piceetalia abietis Pawłowski ex Pawłowski et al. 1928) is also the presence of very numerous group of species which almost exclusively grows only in the former alliance when considering the communities of natural mountain Norway spruce woodlands. This group consists of species which require nutrient-rich habitats, for example Valeriana tripteris, Primula elatior, Polygonatum verticillatum, Phyteuma spicatum, Myosotis sylvatica, Geranium sylvaticum, Heracleum sphondylium, Fragaria vesca, Mercurialis perennis, Lilium martagon, Thalictrum aquilegiifolium, Paris quadrifolia, Dentaria enneaphyllos and many more.

Species common in the so-called acid Norway spruce woodlands, and usually common in lower lying upper montane mixed Fagus woodlands as well, e.g. Oxalis acetosella, Luzula sylvatica ssp. sylvatica, Homogyne alpina, Prenathes purpurea, Dryopteris dilatata, D. expansa, are also abundant in the phytocoenoses of the alliance Cortuso-Piceion. Adenostyles alliariae is a field layer dominant of subunits of the various floristically and ecologically distinct communities. Outspoken calcifuges (Vaccinium myrtillus, Avenella flexuosa, Calamagrostis villosa, V. vitisidaea a pod.) usually reach only low cover values or they are completely missing.

For the ground layer of the Cortuso matthioli-Piceion woodlands presence of species Mnium spinosum, Tortella tortuosa, Ctenidium molluscum, Plagiochila asplenioides, Rhizomnium punctatum is characteristic. Common woodland species Dicranum scoparium, Hylocomium splendens, Plagiothecium curvifolium, Rhytidiadelphus triquetrus, Polytrichum formosum and others are usually present as well.

Nomenclatural note. - The alliance names "Chrysanthemo-Piceion Březina et Hadač in Hadač 1962" as well as
"Oxalido-Piceion Březina et Hadač in Hadač 1962" were used in the previously published regional and international surveys for the respective syntaxon of calcicolous natural Norway spruce woodlands. As Kučera (in red.) extensively explained, the original diagnoses of these syntaxa considerably differ from their traditional use which corresponds to their application as pseudonyma Chrysanthemo-Piceion auct. non (Krajina 1933) Březina et Hadač in Hadač 1962 and Oxalido-Piceion auct. non (Krajina 1933) Březina et Hadač in Hadač 1962.

Therefore new name Cortuso matthioli-Piceion abietis was proposed to replace the mentioned pseudonyma. The proposal to establish such new alliance represent a return to the previous conception of Kučera (2007).

\section{Conclusions}

The second version and at the same time third part of the syntaxonomical revision of the communities traditionally classified within the class Vaccinio-Piceetea Br.-Bl. in Br.-Bl. et al. 1939 from the territory of the Slovak Western Carpathians is presented. It is dedicated to Norway spruce woodlands which are classified within the order Cortuso matthioli-Piceetalia (= Athyrio-Piceetalia auct. non Hadač 1962). The revision is based on careful data selection made in effort to separate the natural supramontane Norway spruce woodlands from the secondary Picea abies phytocoenoses in which evident secondary succession of Fagus sylvatica is in progress and which as such belong to the class Carpino-Fagetea Jakucs ex Passarge 1968. The variety of the Western Carpathian Norway spruce vegetation types found on carbonate rocks in habitat series from the most extreme habitats with shallow soils to more deep soils decalcified in the upper soils horizon is classified within six associations arranged in the following scheme:

Cortuso matthioli-Piceetalia abietis P. Kučera 2022 ord. nov.

Cortuso matthioli-Piceion abietis P. Kučera 2022 all. nov.

1. Seslerio caeruleae-Piceetum abietis Fajmonová 1978 nom. corr. et nom. cons. propos.

2. Cirsio erisithalis-Piceetum abietis Fajmonová et $\mathrm{P}$. Kučera in P. Kučera 2022

3. Fragario vescae-Piceetum abietis P. Kučera 2022 ass. nov. prov.

4. Adenostylo alliariae-Piceetum abietis Samek et al. 1957 nom. corr. et nom. cons. propos.

5. Mnio spinosi-Piceetum abietis Hadač et al. 1969 nom. corr.

6. Hieracio murorum-Piceetum abietis P. Kučera 2022 ass. nov. 


\section{Acknowledgments}

My sincere thanks belong to R. Bouef for valuable help with literature, especially old Fagus- and Abies-syntaxa names as well as to librarians of the Slovak National Library in Martin and to librarians I. Pekárová and I. Gažiová (Institute of Botany SAS, Bratislava). I would like to also thank to J.-P. Theurillat for the various nomenclatural advices provided in the recent years and to Ch. Schaffer of the Revierforstamt Tschiertschen-Praden for confirmation of the existence of Fagus woodland near Tschiertschen (Switzerland). Finally, I would like to thank to the anynomous reviewers for their correction to the manuscript and especially for their inspiring comments.

Peter Kučera (D) https://orcid.org/0000-0002-8508-616X

\section{Funding}

This study was funded by the Slovak grant agency VEGA, project No. 2/0119/19.

\section{References}

Accetto, M. (1993). Mraziščna smrečja (Asplenio-Piceetum R. Kuoch 1954 var. geogr. Omphalodes verna var. geogr. nova) v koliševkah Kočevske. Gozdarski vestnik, 51(10), 426-445.

Adamczyk, B. (1962). Studia głeboznawco-fitosocjologiczne w Dolinie Małej Łaki w Tatrach. Acta Agraria et Silvestria, Seria leśna II: 45-116.

Adorni, M. (2016). La vegetazione legnosa in Emilia. Censimento e analisi delle fitocenosi arboree e arbustive. Istituto per i Beni Artistici Culturali e Naturali della Regione Emilia-Romagna. http://online. ibc.regione.emilia-romagna.it///libri/pdf/La_vegetazione_legnosa_in_ Emilia.pdf

Angellini, P., Bianco, P., Cardillo, A., Francescato, C., \& Oriolo, G. (2009). Gli habitat in Carta della Natura. Schede descrittive degli habitat per la cartografia alla scala 1:50.000. Istituto Superiore per la Protezione e la Ricerca Ambientale. http://www.isprambiente.gov.it/it/ pubblicazioni/manuali-e-linee-guida/gli-habitat-in-carta-della-naturaschede-descrittive-degli-habitat-per-la-cartografia-alla-scala-1-50.000

Bartsch, J., \& Bartsch, M. (1940). Vegetationskunde des Schwarzwaldes. Pflanzensoziologie, 4, 1-234.

Beger, H. K. E. (1922). Assoziationsstudien in der Waldstufe des Schanfiggs. Mitteilungen aus dem Botanischen Museum der Universität Zürich, 46: 1-148.

Bergmeier, E. (2020). Die Vegetation Deutschlands - eine vergleichende Übersicht der Klassen, Ordnungen und Verbände auf Grundlage der EuroVegChecklist. Tuexenia, 40: 19-32. https://doi. org/10.14471/2020.40.024

Biondi, E., Blasi, C., Allegrezza, M., Anzellotti, I., Azzella, M. M., Carli, E., Casavecchia, S., Copiz, R., Del Vico, E., Facioni, L., Galdenzi, D., Gasparri, R., Lasen, C., Pesaresi, S., Poldini, L., Sburlino, G., Taffetani, F., Vagge, I., Zitti, S., \& Zivkovic, L. (2014). Plant communities of Italy: The Vegetation Prodrome. Plant Biosystems, 148(4), 728-814. https://doi.org/10.1080/11263504.2014.948527
Boeuf, R., Simler, N., Holveck, P., Hum, Ph., Cartier, D., \& Ritz, F. (2014). Les végétations forestières d'Alsace. Vol. I. (Textes): Réferentiel des types forestiers du type générique au type élémentaire - Relations entre les stations forestières, les communautés forestières, les habitats et les espèces végétales patrimoniales. Office National de Forêts, Ministère de l'Alimentation, de l'Agriculture et de la Pêche.

Boublík, K., Douda, J., Hédl, R., \& Chytrý, M. (2013). Mezofilní a vlhké opadavé listnaté lesy (Carpino-Fagetea). In M. Chytrý (ed.), J. Douda, J. Roleček, J. Sádlo, K. Boublík, R. Hédl, M. Vítková, D. Zelený, J. Navrátilová, Z. Neuhäuslová, P. Petřík, J. Kolbek, Z. Lososová, K. Šumberová, R. Hrivnák, D. Michalcová, K. Žáková, J. Danihelka, L. Tichý, V. Zouhar, O. Hájek, \& M. Kočí, Vegetace České republiky. 4. Lesní a krovinová vegetace (pp. 193-295). Nakladatelství Academia.

Braun-Branquet, J., Sissingh, G., \& Vlieger, J. (1939). Prodromus der Pflanzengesellschaften: Prodrome des Groupements végétaux. Fasz. 6. Klasse der Vaccinio-Piceetea (Nadelholz- und Vaccinienheiden-Verbände der eurosibirisch-nordamerikanischen Region). Comité International du Prodrome Phytosociologique.

Braun-Blanquet, J., Pallmann, H., \& Bach, R. (1954).

Pflanzensoziologische und bodenkundliche Untersuchungen im Schweizerischen Nationalpark und seinen Nachbarngebieten. II. Vegetation und Böden der Wald- und Zwergstrauchgesellschaften (Vaccinio-Piceetalia). Ergebnisse der wissenschaftlichen Untersuchungen des schweizerischen Nationalparks (Neue Folge), IV, 1-200.

Chifu, T. (2014). Vaccinio-Piceetea Br.-Bl. in Br--Bl. et al. 1939. In T. Chifu (Ed.), \& I. Irimia, Diversitatea fitosociologică a vegetației României. III. Vegetația pădurilor şi tufişurilor (pp. 462-510). Institulul European.

Chytrý, M., Tichý, L., Holt, J., \& Botta-Dukát, Z. (2002a). Determination of diagnostic species with statistical fidelity measures. Journal of Vegetation Science, 13(1), 79-90. https://doi. org/10.1111/j.1654-1103.2002.tb02025.x

Chytrý, M., Exner, A., Hrivnák, R., Ujházy, K., Valachovič, M., \& Willner, W. (2002b). Context-dependence of diagnostic species: A case study of the central european spruce forests. Folia geobotanica, 37(4), 403-417. https://doi.org/10.1007/BF02803255

Chytrý, M. (ed.), Douda, J., Roleček, J., Sádlo, J., Boublík, K., Hédl, R., Vítková, M., Zelený, D., Navrátilová, J., Neuhäuslová, Z., Petř́ík, P., Kolbek, J., Lososová, Z., Šumberová, K., Hrivnák, R., Michalcová, D., Žáková, K., Danihelka, J., Tichý, L., Zouhar, V., Hájek, O., \& Kočí, M. (2013a). Vegetace České republiky. 4. Lesni a křovinová vegetace. Nakladatelství Academia.

Chytrý, M., Zelený, D., Navrátilová, J., \& Sádlo, J. (2013b). Boreokontinentální jehličnaté lesy (Vaccinio-Piceetea). In M. Chytrý (Ed.), J. Douda, J. Roleček, J. Sádlo, K. Boublík, R. Hédl, M. Vítková, D. Zelený, J. Navrátilová, Z. Neuhäuslová, P. Petř́k, J. Kolbek, Z. Lososová, K. Šumberová, R. Hrivnák, D. Michalcová, K. Žáková, J. Danihelka, L. Tichý, V. Zouhar, O. Hájek, \& M. Kočí, Vegetace České republiky. 4. Lesni a krovinová vegetace (pp. 380-432). Nakladatelství Academia.

Coldea, G. (2015). Classe Vaccinio-Piceetea Br.-Bl. in Br.-Bl. et al. 1939. In G. Coldea (Ed.), A. Indreica, \& A. Oprea, Les associations végétales de Roumanie. Tome 3. Les associations forestiéres et arbustives (pp. 198-238). Presa Universitară Clujeană.

Černušáková, D. (1994). The climax spruce stands from Osobitá. Biologia (Bratislava), 49(1), 31-40.

Dierßen, K., \& Dierßen, B. (1996). Vegetation Nordeuropas. Eugen Ulmer. 
Domin, K. (1923). A Phytogeographical Outline of the Zonal Division in the Western Carpathians, besides some general remarks on the main forest trees. Spisy vydávané Prírodovédeckou fakultou Karlovy university, 1, 1-44.

Dubyna, D. V., Dziuba, T. P., Iemelianova, S. M., Bagrikova, N. O., Borysova, O.V., Borsukevych, L. M., Vynokurov, D. S., Gapon, S. V., Gapon, Yu. V., Davydov, D. A., Dvoretskyi, T. V., Didukh, Ya. P., Zhmud, O. I., Kozyr, M. S., Konishchuk, V. V., Kuzemok, A. A., Pashkevich, N. A., Ryff, L. E., Solomakha, V. A., Felbaba-Klushyna, L. M., Fitsailo, T. V., Chorna, G. A., Chorney, I. I., Shelyag-Sosonko, Yu. R., \& Iakushenko, D. M. (2019). Prodromus roslynnosti Ukrajiny. Naukova Dumka.

Eggler, J. (1952). Pflanzendecke des Schöckels. Landesmuseum Joanneum.

Ellenberg, H. (1963). Vegetation Mitteleuropas mit den Alpen in kausaler, dynamischer und historischer Sicht. Eugen Ulmer.

Ellenberg, H. (1996). Vegetation Mitteleuropas mit den Alpen in ökologischer, dynamischer und historischer Sicht (5th ed.). Eugen Ulmer.

Ellenberg, H., \& Klötzli, F. (1972). Waldgesellschaften und Waldstandorte der Schweiz. Mitteilungen der Schweizerischen Anstallt für das forstliche Versuchswesen, 48(4), 587-930.

Exner, A., Willner, W., \& Grabherr, G. (2002). Picea abies and Abies alba forests of the Austrian Alps: Numerical classification and ordination. Folia geobotanica, 37(4), 365-382. https://doi. org/10.1007/BF02803254

Exner, A. (2007). Piceetalia Pawł. 1928. Eurosibirische Fichten- und Fichten-Tannenwälder. In W. Willner, G. Grabherr (Eds), A. Drescher, Ch. Eichberger, A. Exner, R. F. Wilfried, S. Grabner, P. Heiselmayer, P. Karner, F. Starlinger, N. Sauberer, \& G. M. Steiner, Die Wälder und Gebüsche Österreichs: Ein Bestimmungswerk mit Tabellen. 1 Textband (pp. 184-208). Elsevier.

Fajmonová, E. (1978). K syntaxonómii spoločenstiev radu AthyrioPiceetalia Hadač 62 v Západných Karpatoch. Biológia (Bratislava), 33(7), 551-563.

Fajmonová, E. (1983). Cenotické optimum druhov a druhová diferenciácia fytocenóz asociácie Cortuso-Fagetum a Cortuso-Piceetum. Biológia (Bratislava), 38(5), 461-467.

Fajmonová, E. (1986). K variabilite asociácie Cortuso-Piceetum na Slovensku. Preslia, 58(1), 43-54.

Faško, P., Lapin, M., \& Pecho, J. (2008). 20-Year extraordinary climatic period in Slovakia. Meteorologický ćasopis, 11(3): 99-105. http://www.shmu.sk/sk/?page=31

Fischer, M. A. (ed.), Oswald, K., Adler, W., Gottschlich, G., Englmaier, P., Danner, J., Schuhwerk, F., Hörandl, E., Grims, F., Walter, J., Starmühler, W., Saukel, J., Mrkvicka, A. Ch., Speta, F., Wilhalm, Th., Dietrich, G., Greimler, J., Polatschek, A., Willner, W., Teppner, H., Kästner, A., Gregor, Th., Kirschner, J., Zidorn, Ch. H. W., Schmidt, P. A., Schneeweiß, G. M., Zernig, K., Essl, F., Karrer, G., Hohla, M. Stöhr, O., et al. (2008). Exkursionsflora von Österreich, Liechtenstein und Südtirol: Bestimmungsbuch für alle in Republik Österreich, im Fürstentum Liechtenstein und in der Autonomen Provinz Bozen / Südtirol (Italien) wildwachsenden sowie die wichtigsten kultivierten Gefäßpflanzen (Farnpflanzen und Samenpflanzen) mit Angaben über ihre Ökologie und Verbreitung. Land Oberösterreich, Oberösterreichische Landesmuseen.

Fleischer, P., \& Chmiel, J. (2010). Lesy. In A. Koutná, \& B. Chovancová (Eds.), Tatry - Príroda (pp. 279-298). Nakladatelství Miloš Uhlír - Baset.
Foggi, B., Nardi, E., \& Rossi, G. (2001). Nomenclatural notes and typification in Sesleria Scop. (Poaceae). Taxon, 50(4): 1101-1106. https://doi.org/10.2307/1224726

Futák, J. (1972). Činitele pôsobiace na rozšírenie rastlín. In J. Bako, J. Berta, O. Ferianc, Z. Feriancová-Masárová, O. Fusán, J. Futák, S. Hejný, A. Jurko, L. Korbel, M. Kurpelová, M. Lukniš (scientific red.), L. Mičian, J. Michalko, Š. Petrovič, Z. Schmidt, E. Šimo, F. Vilček, \& M. Zatko, Slovensko. Príroda (pp. 408-412). Obzor.

Gentile, S. (1995). Vegetazione a Pinus uncinata Mill. var. rostrata Ant. nella catena montuosa dello spartiacque ligure-emiliano. Fitosociologia, 29, 95-101.

Grebenščikov, O., Michalko, J., Hlaváček, A., Zahradníková, K., \& Brillová, D. (1956). Geobotanický a floristický náčrt Kubínskej Hole. Biologické práce, 2(5), 1-92.

Hadač, E. (1962). Übersicht der höheren Vegetationseinheiten des Tatragebirges. Vegetatio, 9(1-2), 46-54.

Hadač, E. (1965). Poznámky k syntaxonomii karpatských jedlin. Biológia (Bratislava), 20(8), 592-599.

Hadač, E., \& Sofron, J. (1980). Notes on Syntaxonomy of Cultural Forest Communities. Folia geobotanica et phytotaxonomica, 15(3), 245-258. https://doi.org/10.1007/BF02851808

Hadač, E., Březina, P., Ježek, V., Kubička, J., Hadačová, V., Vondráček, M. et al. (1969). Die Pflanzengesellschaften des Tales „Dolina Siedmich prameňov" in der Belaer Tatra. Vydavatel'stvo Slovenskej akadémie vied.

Haeupler, H. (1970). Vorschläge zur Abgrenzung der Höhenstufen der Vegetation im Rahmen der Mitteleuropakartierung. Göttinger Floristische Rundbriefe, 4, 3-15.

Hančinský, L. (1972). Lesné typy Slovenska. Príroda.

Hančinský, L. (1977). Príspevok k rekonštrukcii pôvodného rozšírenia lesných spoločenstiev a ich drevinového zloženia na území Tatranského národného parku na podkladoch lesníckej typológie, histórie a onomastiky. Zborník prác o Tatranskom národnom parku, 19, 97-126.

Hartmann, F. K. (1953). Waldgesellschaften der deutschen Mittelgebirge und des Hügellandes: Nach ihren wichtigsten soziologischen und standörtlichen Merkmalen in einer vorläufigen Übersicht zusammengestellt. Umschaudienst des Forschungsausschusses "Landschaftspflege und Landschaftsgestaltung" der Akademie für Raumforschung und Landesplanung, 4-6, 139-182.

Hartmann, F. K. (1959). Q. Naturnahe Waldgesellschaften Deutschlands in regionaler und standortökologischer Anordnung (mit Ausnahme des Alpengebietes). In R. Müller (Ed.), H. Beyer, A. Bonnemann, J. Braeuer, K. Christopeit, H. Eberts, K. Evers, R. Geiger, H. Gläser, F. K. Hartmann, D. von Hegel, H.-H., Heitmüller, H. H. Hilf, F. Klose, E. Kussmann, W. V. Laer, E. Mammen, H. Mayer-Wegelin, W. Meyer, F. Nüsslein, J. Oelkers, K. von Raben, M. Reinhold, E. Rohmeder, E. Röhrig, H. Schmidt, F. Schwerdtfeger, J. Speer, G. Speidel, A. Freiherr von Vietinghoff-Riesch, K. F. Wentzel, \& W. Wittich, Grundlagen der Forstwirtschaft in Übersicht, Zahl, Tabelle, Regel, Vorschrift, Gesetz (pp. 765-790). M. \& H. Schaper.

Hartmann, F. K., \& Jahn, G. (1967). Ökologie der Wälder und Landschaften. Band 1. Waldgesellschaften des mitteleuropäischen Gebirgsraumes nördlich der Alpen: Tabellen, Grundlagen und Erläuterungen. Gustav Fischer.

Hennekens S. M. (c1998-2020). Turboveg for Windows. International single user version. Ver. 2.149a. [Computer software]. S. M. 
Hennekens, (C) 1998-2020. Comprehensive database management system designed for the storage, selection, and export of vegetation data (relevés). http://www.synbiosys.alterra.nl/turboveg/

Hennekens, S. M., \& Schaminée, J. H. J. (2001). Turboveg, a comprehensive database management system for vegetation data. Journal of Vegetation Science, 12(4), 589-591. https://doi. org/10.2307/3237010

Heß, E., Landolt, E., \& Hirzel, R. (1967). Flora der Schweiz und angrenzender Gebiete. Band I: Pteridophyta bis Caryophyllaceae. Birkäuser Verlag.

Historická ortofotomapa Slovenska (s. d.). Centrum excelentnosti pre podporu rozhodovania v lese a krajine, TU Zvolen, Zvolen. Historická ortofotomapa (C) GEODIS SLOVAKIA, s.r.o., Historické LMS (C) Topografický ústav Banská Bystrica. Retrieved February 22, 2021, from http://mapy.tuzvo.sk/HOFM/.

Holub, J., \& Jirásek, V. (1967). Zur Vereinheitlichung der Terminologie in der Phytogeographie. Folia geobotanica et phytotaxonomica, 2(1), 69-113. https://doi.org/10.1007/BF02851755

Holub, J., Hejný, S., Moravec, J., \& Neuhäusl, R. (1967). Übersicht der höheren Vegetationseinheiten der Tschechoslowakei. Rozpravy Československé akademie véd, Řada matematických a prírodnich véd, 77(3), $1-76$

Horvat, I. (1962). Vegetacija planina Zapadne Hrvatske. Sa 4 karte biljnih zajednica sekcije Sušak. JAZU.

Horvat, I., Glavač, V., \& Ellenberg, H. (1974). Vegetation Südosteuropas. VEB Gustav Fischer.

Issler, E. (1926). Les associations végétales des Vosges méridionales et de la Plaine Rhénane avoisinante. Première Partie: Les forêts (fin). Bulletin de la Société d'histoire naturelle de Colmar, Nouvelle série, 19, $1-109$.

Issler, E. (1931). Les associations silvatiques haut-rhinoises: Classification sociologique des Forêts du département du Haut-Rhin à l'exclusion du Sundgau et du Jura alsacien (avec une carte). Bulletin de la Société Botanique de France, 73(6), 62-141. https://doi.org/10.10 80/00378941.1926.10832847

Issler, E. (1942). Vegetationskunde der Vogesen. Pflanzensoziologie, 5, $1-192$.

Jahn, G. (1977). Die Fichtenwaldgesellschaften in Europa. In H. Schmidt-Vogt, G. Jahn, \& D. Vogellehner, Die Fichte. Band I: Taxonomie Verbreitung Morphologie Ökologie Waldgesellschaften (pp. 468-560). Paul Parey.

Jahn, G. (1985). Chorological phenomena in spruce and beech communities. Vegetatio, 59(1-3), 21-37.

Jalas, J., \& Suominen, J. (Eds.) (1973). Atlas Florae Europaeae. Distribution of Vascular Plants in Europe. 2. Gymnospermae (Pinaceae to Ephedraceae). The Committee for Mapping the Flora of Europe \& Societas Biologica Fennica Vanamo.

Jankovská, V. (1984). Late Glacial Finds of Pinus cembra L. in the Lubovnianská kotlina Basin. Folia geobotanica et phytotaxonomica, 19(3), 323-325. https://doi.org/10.1007/BF02853098

Jankovská, V. (1991). Vývoj vegetačního krytu podtatranských kotlin od konce doby ledové po současnost. Zborník prác o Tatranskom národnom parku, 31, 73-84.
Jankovská, V., Chromy, P., \& Nižnianská, M. (2002). Šafárka - first palaeobotanical data of the character of Last Glacial vegetation and landscape in the West Carpathians (Slovakia). Acta Palaeobotanica, 42(1), 39-50.

Jankovská, V., Baroň, I., Nývlt, D., Krejčí, O., \& Krejčí, V. (2018). Last Glacial to Holocene vegetation succession recorded in polyphase slope-failure deposits on the Maleník Ridge, Outer Western Carpathians. Quaternary International, 470 (Part A), 38-52. https:// doi.org/10.1016/j.quaint.2017.10.048

Jarolímek, I., Šibík, J., Hegedüšová, K., Janišová, M., Kliment, J., Kučera, P., Májeková, J., Michálková, D., Sadloňová, J., Šibíková, I., Škodová, I., Uhlíŕová, J., Ujházy, K., Ujházyová, M., Valachovič, M., \& Zaliberová, M. (2008a). A list of vegetation units of Slovakia. In I. Jarolímek, \& J. Šibík (Eds.), Diagnostic, constant and dominant species of the higher vegetation units of Slovakia (pp. 295-329). VEDA, vydavatel'stvo Slovenskej akadémie vied.

Jarolímek, I., Šibík, J., Tichý, L., \& Kliment, J. (2008b). Diagnostic, constant and dominant species of the higher veegtation units of Slovakia. In I. Jarolímek, J. Šibík (Eds.), K. Hegedüšová, M. Janišová, J. Kliment, P. Kučera, J. Májeková, D. Michálková, J. Sadloňová, I. Šibíková, I. Škodová, J. Uhlî́ová, K. Ujházy, M. Ujházyová, M. Valachovič, \& M. Zaliberová, Diagnostic, constant and dominant species of the higher vegetation units of Slovakia (pp. 9-294). VEDA, vydavatel'stvo Slovenskej akadémie vied.

Jäger, E. J. (Ed.), Arndt, St., Bräutigam, S., Buttler, K. P., Fischer, M. A., Fröhner, S. E., Gerstberger, P., Gutte, P., Hand, R., Hanelt, P., Henker, H., Jage, H., Jeßen, St., Kirschner, J., Krisch, H., Kutzelnigg, H., Müller, F., Pistrick, K., Polatschek, A., Pusch, J., Rostanski, K., Schmidt, P. A., Scholz, H., Stolle, J., Uhlemann, I., Vitek, E., Weber, H. E., Welk, E., Wiegler, G., Wisskirchen, R., Mahn, E.-G., Zech, H., Mörchen, G., Fukarek, L., Stein, B., \& Kästner, A. (2017). Rothmaler - Exkursionsflora von Deutschland. Gefäßptlanzen: Grundband. Springer Spektrum. https://doi.org/10.1007/978-3-662-49708-1

Jeník, J., \& Lokvenc, T. (1962). Die alpine Waldgrenze im Krkonoše Gebirge. Rozpravy Československé akademie véd, Rada matematických a prírodnich vèd, 72(1), 1-68.

Jirásek, J. (2002). Tř́da: Vaccinio-Piceetea Br.-Bl. in Braun-Blanquet, Sissingh et Vlieger 1939. In M. Husová, J. Jirásek, \& J. Moravec (Eds.), Přehled vegetace České republiky. Svazek 3. Jehličnaté lesy (pp. 18-86). Academia.

Juvan, N., Košir, P., Marinšek, A., Paušič, A., \& Čarni, A. (2013). Differentiation of the Piceetalia and Athyrio-Piceetalia forests in Slovenia. Tuexenia, 33, 25-48.

Kanka, R. (2008). Lesy Belianskych Tatier. VEDA, vydavatel'stvo Slovenskej akadémie vied.

Kaplan, Z. (Ed.-in-chief), Danihelka, J., Chrtek, J., jun., Kirschner, J., Kubát, K., Štech, M., Štěpánek, J. (Eds.), Batoušek, P., Bureš, P., Businský, R., Čáp, J., Dančák, M., Ducháček, M., Duchoslav, M., Dvořák, V., Ekrt, L., Filippov, P., Grulich, V., Hrčka, D., Hroneš, M., Hrouda, L., Hroudová, Z., Jehlík, V., Kabátová, K., Király, G., Kirschnerová, L., Kobrlová, L., Kočí, K., Koutecký, P., Krahulec, F., Kúr, P., Lepší, M., Mandák, B., Ponert, J., Prančl, J., Pyšek, P., Řepka, R., Sádlo, J., Suda, J., Šída, O., Šmarda, P., Šprynař, P., Štěpánková, J., Trávníček, B., Trávníček, P., Uher, J., Vašut, R. J., Větvička, V., Zázvorka, J., Zelený, V., Skoumalová, A., \& Smrčinová, E. (2019). Klič ke květeně České republiky (2nd ed.). Academia.

Kielland-Lund, J. (1981). Die Waldgesellschaften SO-Norwegens. Phytocoenologia, 9(1/2), 53-250. 
Klika, J. (1926). Poznámky ke geobotanickému výzkumu Velké Fatry. Sborník Prírodovédecké společnosti v M. Ostravě, 3(1924-25), 38-85.

Klika, J. (1929). Lesní typy v rámci našeho lesního geobotanického prozkumu a jejich vztah k lesnictví. Sborník Československé akademie zemédèlské, 4, 229-284.

Klika, J. (1936). Das Klimax-Gebiet der Buchenwälder in den Westkarpathen. Beihefte zum Botanischen Centralblatt, Abteilung B., Systematik, Pflanzengeographie, 55, 373-418.

Klika, J. (1944). B. Společenstva jevnosnubných rostlin. In J. Klika, \& E. Hadač (Eds.), Rostlinná společenstva střední Evropy. Príroda, 36(8), 257-259 \& 36(9), 281-295.

Kobzáková, D. (1987). Fytocenologicko-ekologické pomery klimaxových smrečín pravých svahov Tichej doliny v Západných Tatrách. [Unpublished Master’s thesis]. Prírodovedecká fakulta Univerzity Komenského.

Kočický, D., \& Ivanič, B. (2011). Geomorfologické členenie Slovenska [online]. Štátny geologický ústav Dionýza Štúra. Retrieved February 22, 2021, from http://apl.geology.sk/temapy/

Körner, C. (2012). Alpine Treelines: Functional Ecology of the Global High Elevation Tree Limits. Springer. https://doi.org/10.1007/978-30348-0396-0

Krajčí, J. (2008). Smrečiny severovýchodnej časti Nízkych Tatier okolie Vel'kého Boku. Phytopedon, 7(1-2), 94-103.

Krajčí, J. (2009). Lesné spoločenstvá okolia Velkého Boku. [Unpublished doctoral dissertation]. Ústav krajinnej ekológie SAV.

Krajčí, J., \& Barančok, P. (2009). Lesné spoločenstvá okolia Velkého Boku. In P. Turis, \& L. Vidlička (Eds), Priroda Nizkych Tatier, 2 (pp. 77-85). Správa národného parku Nízke Tatry.

Krajina, V. (1933). Die Pflanzengesellschaften des Mlynica-Tales in den Vysoké Tatry (Hohe Tatra): Mit besonderer Berücksichtigung der ökologischen Verhältnisse. II. Teil. Beihefte zum Botanischen Centralblatt, Abt. II, 51(1), 1-224.

Krippel, E. (1963). Postglaciálny vývoj lesov Tatranského národného parku. Biologické práce, 9(5), 1-44.

Kubát, K. (Ed.-in-chief and ed.), Hrouda, L., Chrtek, J., jun., Kaplan, Z., Kirschner, J., Štěpánek, J. (Eds.), Bělohlávková, R., Bureš, P., Businský, R., Čáp, J., Danihelka, J., Dostálek, J., jun., Drábková, L., Filippov, P., Grulich, V., Havlíček, P., Hrčka, D., Hrouda, L., Hroudová, V., Hroudová, Z., Chrtek, J., sen., Jehlík, V., Kirschnerová, L., Kobližek, J., Kočí, K., Kovanda, M., Krahulec, F., Kř́sa, B., Kúr, P., Mandák, B., Marhold, K., Plocek, A., Procházka, F., Pyšek, P., Řepka, R., Slavík, B., Slavíková, Z., Suda, J., Sída, O., Šmarda, P., Štech, M., Stěpánková, J., Trávníček, B., Větvička, V., Zázvorka, J., Zelený, V., \& SkoumalováHadačová, A. (2002). Klič ke kvètené České republiky. Academia.

Kubinská, A. (Ed.) \& Janovicová, K. (1998). Machorasty. In K. Marhold, \& F. Hindák (Eds.), Zoznam nižšich a vy̌šich rastlin Slovenska (pp. 297-331). VEDA, vydavatel'stvo Slovenskej akadémie vied.

Kubíček, F., \& Jurko, A. (1975). Waldgesellschaften des östlichen Orava-Gebietes. Biologické práce, 21(3). 83-128.

Kubíček, F., Šomšák, L., Šimonovič, V., \& Szabo, J. (1992). Produkčno-ekologická a fytocenologická charakteristika bylinnej vrstvy lesných ekosystémov troch tatranských dolín (Furkotská, Siedmich prameňov, Zadné Medodoly). Zborník prác o Tatranskom národnom parku, 32, 273-305.
Kubíček, F., Šimonovič, V., Minarčic, P., \& Šomšák, L. (1996). Produkčná analýza bylinnej vrstvy niektorých menej zastúpených vápencových smrečín a jedlín Tatranského národného parku. Štúdie o Tatranskom národnom parku, 1(34), 89-108.

Kučera, P. (2002). Lesné spoločenstvá Belianskej doliny vo Vel'kej Fatre. [Unpublished Master's thesis]. Prírodovedecka fakulta Univerzity Komenského.

Kučera, P. (2007). Západokarpatské smrečiny: Komentovaný literárny prehlad. [Spruce woodlands of the Western Carpathians: A commented literature survey]. [Unpublished doctoral dissertation]. Botanický ústav Intitute of botany SAS.

Kučera, P. (2010a). Nomenclatural types of Picea abies syntaxa reported from Slovakia. Biologia (Bratislava), 65(5), 832-836. https://doi. org/10.2478/s11756-010-0103-x

Kučera, P. (2010b). Remarks to Abietion albae and its syntaxa. Acta botanica Universitatis Comenianae, 45, 3-12.

Kučera, P. (2010c). Smrečiny Vel'kej Fatry a pril'ahlých pohorí. [Unpublished doctoral dissertation]. Botanický ústav Intitute of botany SAS.

Kučera, P. (2011a). Use of descriptive statistics in phytosociology. Acta Botanica Universitatis Comenianae, 46, 63-74.

Kučera, P. (2011b). O pôvodnosti smrečín Pol'any. Bulletin Slovenskej botanickej spoločnosti, 33(2), 199-219.

Kučera, P. (2012a). Vegetačný stupeñ smrečín v Západných Karpatoch - rozširenie a spoločenstvá: Spis so zvlástnym zretelom na pohorie Vel'ká Fatra. Botanická záhrada UK v Bratislave.

Kučera, P. (2012b). Remarks on the intramontane continentality of the Western Carpathians defined by the absence of Fagus sylvatica. Thaiszia - Journal of Botany, 22(1), 65-82.

Kučera, P. (2012c). Zhodnotenie údajov o rozšírení stupňa smrečín v Spišskej Magure a poznámky k výskytu jedlín. Naturae tutela, 16(1), $11-26$.

Kučera, P. (2013a). Horské bukové lesy v Západných Karpatoch. [2.2] Veterné hole, skupina Hornej lúky. Natura Carpatica, 56, 17-34.

Kučera, P. (2013b). Two notes to syntaxa names stemmed from Polish geobotanical studies. Hacquetia, 12(1), 133-140. https://doi. org/10.2478/HACQ-2013-0004

Kučera, P. (2014a). Horské bukové lesy v Západných Karpatoch. 2.1 Veterné hole, masív Vel'kej lúky. Natura Carpatica, 55, 39-68.

Kučera, P. (2014b). Jedlové a jedl'ovo-smrekové lesy na geobotanickej mape Slovenska. Bulletin Slovenskej botanickej spoločnosti, 36(1), 65-78.

Kučera, P. (2015a). Errata k článkom o horských bukových lesoch v Západných Karpatoch. Natura Carpatica, 56, 109-114.

Kučera, P. (2015b). On the Occurrence of Natural Norway Spruce Woodland in the Pieniny Mts (Western Carpathians). Acta Silvatica \& Lignaria Hungarica, 11(2), 123-138. https://doi.org/10.1515/aslh2015-0010

Kučera, P. (2017). Two groups of Pinus cembra forest communities in the Tatras. Acta Botanica Hungarica, 59, 389-425. https://doi. org/10.1556/034.59.2017.3-4.7 
Kučera, P. (2019a). Syntaxonomical classification of wet woodlands with Picea abies in Slovakia. Ukrainian Botanical Journal, 76(4), 316-343. https://doi.org/10.15407/ukrbotj76.04.316

Kučera, P. (2019b). Pinus cembra communities in the Tatras comments to the study of Zięba et al. Tuexenia, 39. 161-180. https:// doi.org/10.14471/2019.39.013

Kučera, P. (in prep.). Prehl'ad spoločenstiev triedy Vaccinio-Piceetea Br.Bl. in Br.-Bl. et al. 1939 na Slovensku. Msc., in prep.

Kučera, P. (in red.). On the pseudonymous syntaxonomical application of the order Athyrio-Piceetalia Hadač 1962. In red.

Kučera, P., \& Barančok, P. (2021). Contribution to knowledge on the variability of Arolla pine woodlands of the northeastern Tatra Mountains. Contribuții Botanice, LVI, 29-43. https://doi. org/10.24193/Contrib.Bot.56.3

Kučera, P., \& Kliment, J. (2011). On the nomenclature and syntaxonomy of the phytosociological survey "Die Wälder und Gebüsche Österreichs": examples of the class Piceetea excelsae Klika 1948. Thaiszia - Journal of Botany, 21(2), 85-92.

Kučera, P., Bernátová, D., \& Obuch, J. (2009). Demänovská dolina bezbuková? Naturae tutela, 13(1), 31-42.

Kulczyński, S. (1928). Zespoły roślin w Pieninach. - Die Pflanzenassoziationen der Pieninen. Bulletin International de l'Académie Polonaise des Sciences et des Lettres, Classe des Sciences Mathématiques et Naturelles, Série B., Sciences Natureles, 1928(Suppl. II., 1927), 57-203.

Kuoch, R. (1954). Wälder der Schweizer Alpen im Verbreitungsgebiet der Weißtanne. Mitteilungen der Schweizerischen Anstallt für das forstliche Versuchswesen, 30, 133-260.

Lakatosová, A. (1971). Fytocenologické pomery asociácie Adenostylo-Piceetum (Sill. 33) comb. nova v centrálnokarpatskej oblasti. In L. Dzubinová, E. Fajmonová, A. Lakatosová, P. Pitoniak, J. Šimeková, R. Šoltés, \& E. Véghová, Syntaxonomická charakteristika niektorých lesných spoločenstiev s pribliadnutím k výskytu drubu Sorbus aucuparia $L$. na Slovensku (pp. 230-231). [s. n.].

Lapin, M., Štastný, P., \& Chmelík, M. (2005). Detection of climate change in the Slovak mountains. Hrvatski meteorološki časopis, 40(40), 101-104. https://hrcak.srce.hr/hmc

Lauber, K., Wagner, G., \& Gygax, A. (2018). Flora Helvetica. Haupt Verlag.

Löve, D. (1970). Subarctic and Subalpine: Where and What? Arctic and Alpine Research, 2(1), 63-73. https://doi.org/10.2307/1550141

Marhold, K. (Ed.), Goliašová, K., Hegedüšová, Z., Hodálová, I., Jurkovičová, V., Kmetová, E., Letz, R., Michalková, E., Mráz, P., Peniašteková, M., Šípošová, H., Tavoda, O. (1998). Papradorasty a semenné rastliny. In K. Marhold, \& F. Hindák (Eds.), Zoznam nižšich a vyššich rastlin Slovenska (pp. 333-687). VEDA, vydavatel'stvo Slovenskej akadémie vied.

Matuszkiewicz, J. (1977). Przegląd fitosocjologiczny zbiorowisk leśnych Polski. Cz. 4. Bory świerkowe i jodłowe. Phytocoenosis, 6(3), 151-226.

Matuszkiewicz, W. (1981). Przewodnik do oznaczania zbiorowisk roślinnych Polski. Państwowe Wydawnictwo Naukowe.

Matuszkiewicz, W. (1984). Die Karte der potenziellen natürlichen Vegetation von Polen. Braun-Blanquetia, 1, 1-100.
Matuszkiewicz, W. (2014). Przewodnik do oznaczania zbiorowisk roślinnych Polski. Wydawnictwo Naukowe PWN.

Mayer, H. (1974). Ökologie der Wälder und Landschaften. Band 3. Wälder des Ostalpenraumes: Standort, Aufbau und waldbauliche Bedeutung der wichtigsten Waldgesellschaften in den Ostalpen samt Vorland. Gustav Fischer.

Mayer, H. (1984). Wälder Europas. Gustav Fischer.

Medwecka-Kornaś, A. (1972). Zespoły leśne i zaroślowe. In W. Szafer, K. Zarzycki (Eds.), A. Medwecka-Kornaś, J. Kornaś, S. Pawłowska, B. Pawłowski, A. Środoń, \& A. Kozłowska, Szata roślinna Polski. Tom I (pp. 383-441). Państwowe Wydawnictwo Naukowe.

Mered’a, P., Majerová, M, Somlyay, L., Pekárik, L., \& Hodálová, I. (2019). Genome size variation in the Western Carpathian Sesleria (Poaceae) species. Plant Systematics and Evolution, 305(10), 845-864. https://doi.org/10.1007/s00606-019-01622-1

Meusel, H., Jäger, E., \& Weinert, E. (1965). Vergleichende chorologie der zentraleuropäischen Flora. Text. VEB Gustav Fischer Verlag.

Miadok, D. (1995). Vegetácia ŠPR Ďumbier. Univerzita Komenského v Bratislave.

Michalko, J., \& Berta J. (1972). Lesné spoločenstvá. In J. Bako, J. Berta, O. Ferianc, Z. Feriancová-Masárová, O. Fusán, J. Futák, S. Hejný, A. Jurko, L. Korbel, M. Kurpelová, M. Lukniš, (Scientific red.), L. Mičian, J. Michalko, Š. Petrovič, Z. Schmidt, E. Šimo, F. Vilček, \& M. Zatko, Slovensko. Príroda (pp. 486-531). Obzor.

Michalko, J., Berta, J., Magic, D., \& Maglocký, Š. (1980). Potenciálna prirodzená vegetácia. In: Atlas Slovenskej socialistickej republiky. Slovenská akadémia vied. Slovenský úrad geodézie a kartografie.

Michalko, J. (Ed.), Berta, J., \& Magic, D. (1986). Geobotanická mapa ČSSR. Slovenská socialistická republika. Textová čast' a mapy. Veda.

Mindáš, J. (1999). Vertical climatic ranges of forest trees in Western Carpathian region. Acta instituti forestalis Zvolen, 9, 29-41.

Mirek, Z., \& Piękoś-Mirkowa, H. (1992). Plant cover of the Western Carpathians. Veröffentlichungen des Geobotanischen Institutes der ETH, Stiftung Rübel, 107, 116-150.

Moravec, J., Husová, M., \& Neuhäuslová, Z. (2000). Fagetalia sylvaticae Pawłowski in Pawłowski, Sokołowski et Wallisch 1928. In J. Moravec (Ed.), M. Husová, M. Chytrý, \& Z. Neuhäuslová, Přebled vegetace České republiky. Svazek 2, Hygrofilni, mezofilni a xerofilni opadavé lesy (pp. 14-201). Academia.

Societas pedologica Slovaca (2014). Morfogenetický klasifikačný systém pôd Slovenska: bazálna referenčná taxonómia (2nd ed.). NPPC Výskumný ústav pôdoznalectva a ochrany pôdy.

Mucina, L., Maglocký, Š. (Eds.), Balátová-Tuláčková, E., Banásová, V., Berta, J., Dúbravcová, Z., Fajmonová, E., Hadač, E., Hejný, S., Jarolímek, I., Kontriš, J., Krahulec, F., Krippelová, T., Michalko, J., Michalko, M., Neuhäusl, R., Otahelová, H., Paclová, L., Petrík, A., Rybníček, K, Ščepka, A., Šomšák, L., Špániková, A, Uhlírová, J., \& Zaliberová, M. (1985). A list of vegetation units of Slovakia. Documents phytosociologiques N. S., 9, 175-220.

Mucina, L., Bültmann, H., Dierßen, K., Theurillat, J., Raus, T., Čarni, A., Šumberová, K., Willner, W., Dengler, J., García, R. G., Chytrý, M., Hájek, M., Di Pietro, R., Iakushenko, D., Pallas, J., Daniëls, F. J., Bergmeier, E., Santos Guerra, A., Ermakov, N., Valachovič, M., Schaminée, J. H., Lysenko, T., Didukh, Y. P., Pignatti, S., Rodwell, J. 
S., Capelo, J., Weber, H. E., Solomeshch, A., Dimopoulos, P., Aguiar, C., Hennekens, S. M., \& Tichý, L. (2016). Vegetation of Europe: hierarchical floristic classification system of vascular plant, bryophyte, lichen, and algal communities. Applied Vegetation Science, 19(Suppl. 1), 3-264. https://doi.org/10.1111/avsc.12257

Neuhäusl, R. (1994). Vegetační mapování. In J. Moravec, D. Blažková, S. Hejný, M. Husová, J. Jeník, J. Kolbek, F. Krahulec, V. Krečmer, Z. Kropáć, R. Neuhäusl, Z. Neuhäuslová-Novotná, K. Rybníček, E. Rybničková, V. Samek, \& J. Štěpán, Fytocenologie (Nauka o vegetaci) (pp. 306-322). Academia.

Neuhäuslová, Z. Blažková, D., Grulich, V., Husová, M., Chytrý, M. Jeník, J., Jirásek, J., Kolbek, J., Kropáč, Z., Ložek, V., Moravec, J., Prach, K., Rybníček, K., Rybníčková, E., \& Sádlo, J. (2001). Mapa potenciální prirozené vegetace České republiky. Academia.

Neuhäuslová-Novotná, Z. (1994). Rozširíení rostlinných společenstev a jejich funkce v krajině. In J. Moravec, D. Blažková, S. Hejný, M. Husová, J. Jeník, J. Kolbek, F. Krahulec, V. Krečmer, Z. Kropáč, R. Neuhäusl, Z. Neuhäuslová-Novotná, K. Rybníček, E. Rybníčková, V. Samek, \& J. Štěpán, Fytocenologie (Nauka o vegetaci) (pp. 277-305). Academia.

Nižnanská, M. (1983). Beitrag zur Rekonstruktion der Waldbestände im östlichen Teil des Gebirges Slovenské Rudohorie mit Hilfe der Holzkohlenanalyse. Acta Facultatis rerum naturalium Universitatis Comenianae, Botanica, 30, 65-87.

Oberdorfer, E. (1957). Süddeutsche Pflanzengesellschaften. Pflanzensoziologie, 10, 1-564.

Pawłowski, B. (1928). Guide des excursions en Pologne. I. Partie. Guide de l'excursion botanique dans les Monts Tatras (Environs du lac "Morskie Oko" et du massif des „Czerwone Wierchy): Accompagné de le caractéristique géobotanique générale des Tatras. Orbis.

Pawłowski, B. (1956). Flora Tatr: Rośliny naczyniowe. Tom I. Państwowe wydawnictwo naukowe.

Pignatti, S. (1998). La foresta boreale di conifere. In S. Pignatti, A.Bartoli, C. Blasi, G. Bolognini, F. Clauser, M. Codogno, M. De Lillis, G. Dowgiallo, A. Lapresa, P. L. Nimis, S. Piersanti, G. Pignatti, \& P. Rombolà, I boschi d'Italia: Sinecologia e biodiversità (pp. 125169). UTET.

Pignatti, S., \& Pignatti, E. (2014). Plant Life of the Dolomites: Vegetation Structure and Ecology. Springer. https://doi. org/10.1007/978-3-642-31043-0

Pignatti, S., Guarino, R., \& La Rosa, M. (Eds.) (2017). Flora d'Italia. 1. Edagricole.

Pišút, I. (ed.), Guttová, A., Lackovičová, A., \& Lisická, E. (1998). Lichenizované huby (lišajníky). In K. Marhold, \& F. Hindák (Eds.), Zoznam nižšich a vyšsich rastlin Slovenska (pp. 229-295). VEDA, vydavatel'stvo Slovenskej akadémie vied.

Plesník, P. (1956). Horná hranica lesa v Krivánskej Malej Fatre. Lesnicky časopis Slovenskej akadémie vied, 2(2), 97-123.

Plesník, P. (1961). Všeobecná charakteristika Slovenska. In M. Lukniš, \& P. Plesník, Nižiny, kotliny a pohoria Slovenska (pp. 7-38). Osveta.

Plesník, P. (1966). Horná hranica lesa na Velkom Choči. Geografický časopis, 18(1), 56-76.

Plesník, P. (1971). Horná hranica lesa vo Vysokých a Belanských Tatrách. Vydavatel'stvo Slovenskej akadémie vied.
Plesník, P. (1975). Horná hranica lesa v Lúčanskej Malej Fatre. Zborník Pedagogickej fakulty Univerzity Komenského v Bratislave so sídlom $v$ Trnave, Prírodné vedy, Geografia, 4, 103-130.

Plesník, P. (1978). The Upper Timberline in the Vel'ká (Great) Fatra Mountain. Acta Facultatis rerum naturalium Universitatis Comenianae, Geographica, 16, 7-56.

Plesník, P. (1995). Fytogeografické (vegetačné) členenie Slovenska. Geografický časopis, 47(3), 149-181.

Plesník, P. (2004). Vseobecná biogeografia. Univerzita Komenského v Bratislave.

Podani, J. (2001a). SYNTAX 2000 [Computer software]. J. Podani, Budapest. Multivariate Data Analysis Package. (C) J. Podani. http:// podani.web.elte.hu/SYN2000.html

Podani, J. (2001b). SYN-TAX 2000: Computer Program for Data Analysis in Ecology and Systematics. User's Manual. Scientia Publishing.

Pokorný, P., Jankovská, V., \& Horáček, I. (2015). České Hercynikum versus Západní Karpaty: Klíčové biogeografické rozhraní Evropy v posledním glaciálu. Zprávy České botanické společnosti, 50(2), 165-180.

Polák, M., Bujnovský, A., Kohút, M. (Eds.), Filo, I., Pristaš, J., Havrila, M., Vozár, J., Mello, J., Rakús, M., Buček, S., \& Lexa, J. (1997). Geologická mapa Velkej Fatry. Ministerstvo životného prostredia Slovenskej republiky.

Pott, R. (1992). Die Pflanzengesellschaften Deutschlands. Eugen Ulmer.

QGIS.org (2021). QGIS Geographic Information System. QGIS Association. http://www.qgis.org

Randuška, D. (1986). Typologická klasifikácia lesov v SSR. In D. Randuška, J. Vorel, \& K. Plíva Fytocenológia a lesnicka typológia (pp. 143-220). Príroda.

Rastlinné spoločenstvá Slovenska (1995-). VEDA, vydavatel'stvo Slovenskej akadémie vied, Bratislava.

Renaux, B., Timbal, J., Gauberville, Ch., Thébaud, G., Bardat, J., Lalanne, A., Royer, J.-M., \& Seytre, L. (2019). Contribution au Prodrome des végétations de France: les Carpino betuli-Fagetea sylvaticae Jakucs 1967. Documents Phytosociologiques, 11, 1-424.

Rivas-Martínez, S. (1968). Estudio fitosociológico de los bosques y matorrales pirenaicos del piso subalpino. Publicaciones del Instituto de biologia aplicada, 44, 5-44.

Rivas-Martínez, S., Fernández-González, F., Loidi, J., Lousã, M., \& Penas, A. (2001). Syntaxonomical checklist of vascular plant communities of Spain and Portugal to association level. Itinera Geobotanica, 14, 5-341.

Rivas-Martínez, S., Asensi, A., Díez-Garretas, B., Molero, J., Valle, F., Cano, E., Costa, M., Villar, L., Díaz, T. E., Prieto, J. A. F., Llorens, L., del Arco, M., Fernández, F., Sánchez-Mata, D., Penas, Á., Herrero, L., del Río, S., Masalles, R., Ladero, M., Amor, Á., Izco, J., Amigo, J., Loidi, J., Navarro, G., Cantó, P., Alcaraz, F., Báscones, J. C., \& Soriano, P. (2011). Mapa de series, geoseries y geopermaseries de vegetación de España [Memoria del mapa de vegetación potencial de España, 2011]. Parte II. Itinera Geobotanica, 18(1), 1-424.

Rothmaler, W. (1950). Allgemeine Taxonomie und Chorologie der Pflanzen: Grundzüge der speziellen Botanik. Wilhelm Gronau. 
Rothmaler, W. (1955). Allgemeine Taxonomie und Chorologie der Pflanzen: Grundzüge der speziellen Botanik(2nd ed.). Wilhelm Gronau.

Samek, V., Jančařík, V., Kriesl, A., \& Materna, J. (1957). Lesní společenstva severního úbočí Vysokých Tater (Č́st I. Javorová dolina). Lesnicky časopis, 3(1), 3-38.

Sauberer, N., \& Willner, W. (2007). Kurze Einführung in die Naturund Landschaftsgeschichte Österreichs. In W. Willner, G. Grabherr (Eds.), A. Drescher, Ch. Eichberger, A. Exner, R. F. Wilfried, S. Grabner, P. Heiselmayer, P. Karner, F. Starlinger, N. Sauberer, \& G. M. Steiner, Die Wälder und Gebüsche Österreichs: Ein Bestimmungswerk mit Tabellen. 1 Textband (pp. 19-25). Elsevier.

Schmider, P., \& Burnand, J. (1988). Waldgesellschaften im Fürstentum Liechtenstein. Kommentar zur vegetationskundlichen Kartierung der Wälder. Regierung des Fürstentums Liechtenstein.

Seibert, P. (1992). Klasse: Vaccinio-Piceetea Br.-Bl. in Br.-Bl. et al. 39. In Th. Müller, E. Oberdorfer, \& P. Seibert, Süddeutsche Pflanzengesellschaften. Teil IV. Wälder und Gebüsche. A. Textband (pp. 53-80). Gustav Fischer.

Sillinger, P. (1933). Monografická studie o vegetaci Nizkých Tater. Sbor pro výzkum Slovenska a Podkarpatské Rusi.

Svoboda, P. (1939). Lesy Liptovských Tater: Studie o dřevinách a lesních společenstvech se zvláštním zřetelem k vlivům antropozoickým. Opera Botanica Čechica, 1, 1-164.

Stanová, V., Valachovič, M. (eds), Šeffer, J., Lasák, R., Galvánek, D., Dražil., T. Jarolímek, I., Zaliberová, M., Otahel'ová, H., Maglocký, Š., Kliment, J., Dúbravcová, Z., Uhliarová, E., Hrivnák, R., Ujházy, K., Petrík, A., Uhlírová, J., Bernátová, D., \& Dítě, D. (2002). Katalóg biotopov Slovenska. DAPHNE - Inštitút aplikovanej ekológie.

Sýkora, T. (1971). Lesní rostlinná společenstva Jizerských hor. Knižnice Jizerských hor, 11, 1-60.

Szafer, W., Pawłowski, B., \& Kulczyński, S. (1923). Die Pflanzenassoziationen des Tatra-Gebirges 1. Teil: Die Pflanzenassoziationen des Chochołowska-Tales. Bulletin International de l'Académie Polonaise des Sciences et des Lettres, Classe des Sciences Mathématiques et Naturelles, Série B, Sciences Natureles, 1923 (Suppl. 1923), 1-65.

Szafer, W., Kulczyński, S., Pawłowski, B., Stecki, K., \& Sokołowski, M. (1927). Pflanzenassoziationen des Tatra-Gebirges: III, IV und V Teil, (Tafeln 1-12). Bulletin International de l'Académie Polonaise des Sciences et des Lettres, Classe des Sciences Mathématiques et Naturelles, Série B, Sciences Naturelles 1927 (Suppl. 2, 1926), 1-144.

Šály, R. (1986). Pôdne pomery. In K.Vestenický, I. Vološčuk, I. (Supervisors), Z. Ambros, D. Bernátová, J. Bohuš, A. Bujnovský, A. Čaputa, J. Darola, Š. Fekete, M. Gajdoš, J. Galvánek, J. Kadlečík, J. Kliment, I. Kristek, R. Lacko, J. Lazebníček, V. Ložek, R. Midriak, P. Mitter, V. Peciar, B. Piskún, I. Pišút, M. Polák, V. Stockmann, J. Svatoň, R. Šály, K. Škovirová, \& Z. Žuffová, Velká Fatra: Chránená krajinná oblast (pp. 56-68). Príroda.

Šibík, J. (2012). Slovak Vegetation Database. Biodiversity \& Ecology, 4, 429. https://doi.org/10.7809/b-e.00216.

Šilc, U., \& Čarni, A. (2012). Conspectus of vegetation syntaxa in Slovenia. Hacquetia, 11(1), 113-164.

Šimurdová, B. (2001). Sekundárne smrekové lesy v povodí Hnilca. Bulletin Slovenskej botanickej spoločnosti, 23, 141-147.

Školek, J. (1995a). Rastlinné spoločenstvá v ŠPR Demänovská dolina v Nízkych Tatrách. Naturae tutela, 3, 77-100.
Školek, J. (1995b). The association Carici albae-Piceetum, a new forest community from the Western Carpathians. Oecologia Montana, 4, 41-48.

Školek, J. (2003). Vegetácia Národnej prírodnej rezervácie Ďumbier v Nízkych Tatrách. Naturae tutela, 7, 17-29.

Šmarda, J. et al. (1971). K ekologii rostlinných společenstev Doliny Sedmi pramenů v Belanských Tatrách. Práce a štúdie Československej ochrany prírody, Séria III, 4, 1-208.

Šltés, R. (1969). Sorbus aucuparia L. vo Vysokých Tatrách. [Unpublished Master's thesis]. Prírodovedecká fakulta Univerzity Komenského.

Šoltés, R. (1976). Phytozönotische Analyse des Verbandes Vaccinio-Piceion Br.-Bl. 1938 in den Westkarpaten. Acta Facultatis rerum naturalium Universitatis Comenianae, Botanica, 24, 139-167.

Šomšák, L. (1998). Flóra a fauna v rastlinných spoločenstvách strednej Európy: (Aplikovaná biocenológia). AP.

Thébaud, G., Roux, C., Bernard, Ch.-É., \& Delcoigne, A. (2014). Guide d'identification des végétations du nord du Massif central: Associations végétales et habitat naturels. Presses universitaires Blaise Pascal.

Theurillat, J.-P., Willner, W., Fernández-González, F., Bültmann, H., Čarni, A., Gigante, D., Mucina, L., \& Weber, H. (2021). International Code of Phytosociological Nomenclature. $4^{\text {th }}$ edition. Applied Vegetation Science, 24, Article12491. https://doi.org/10.1111/ avsc. 12491

Tichý, L. (c1998-2020). JUICE. Ver. 7.1.25. [Computer software]. L. Tichý, Brno. Program for analysis and classification of phytosociological tables and other quantitative ecological data sets. http://www.sci.muni.cz/botany/juice/

Tichý, L. (2002). JUICE, software for vegetation classification. Journal of Vegetation Science, 13(3), 451-453. https://doi. org/10.1111/j.1654-1103.2002.tb02069.x

Tichý, L., \& Chytrý, M. (2006). Statistical determination of diagnostic species for site groups of unequal size. Journal of Vegetation Science, 17(6), 809-818. https://doi.org/10.1111/j.1654-1103.2006.tb02504.x

Tison, J.-M., \& de Foucault, B. (Eds.) (2014). Flora Gallica: Flore de France. Biotope.

Tüxen, R. (1955). Das System der nordwestdeutschen Pflanzengesellschaften. Mitteilungen der Floristisch-soziologischen Arbeitsgemeinschaft N. F., 5, 155-176.

Tüxen, R. (1956). Die heutige potentielle natürliche Vegetation als Gegenstand der Vegetationskartierung. Angewandte Pflanzensoziologie, $13,3-42$.

Uhlí̌ová, J., \& Bernátová, D. (1986). Nové lokality bradáčika srdcovitého (Listera cordata (L.) R. Br.) vo Vel'kej Fatre a Slovenskom raji. Biológia (Bratislava), 41(5), 495-497.

Ujházyová, M., Ujházy, K., Máliš, F., Slezák, M., \& Hrivnák, R. (2021) Syntaxonomical revision of the order Fagetalia sylvaticae Pawłowski ex Pawłowski et al. 1928 in Slovakia. Biologia (Bratislava), 76(7), 1929-1968. https://doi.org/10.2478/s11756-020-00661-1

Unar, J., Unarová, M., \& Šmarda, J. (1984). Vegetační poměry Tomanovy doliny a Žlebu spod Diery v Západních Tatrách: Část 1. Fytocenologické tabulky. Folia Facultatis scientiarum naturalium Universitatis Purkynianae Brunensis, Biologia 35(10), 1-104. 
Valachovič, M., Štubňová, E., Senko, D., Kochjarová, J., \& Coldea, Gh. (2019). Ecology and species distribution pattern of Soldanella sect. Soldanella (Primulaceae) within vegetation types in the Carpathians and the adjacent mountains. Biologia (Bratislava), 74(7), 733-750. https://doi.org/10.2478/s11756-019-00200-7

Valachovič, M. et al. (msc.) Rastlinné spoločenstvá Slovenska. 6. Lesná a krovinová vegetácia. VEDA, vydavatel'stvo Slovenskej akadémie vied. In prep.

Viceníková, A., \& Polák, P. (Eds.) (2003). Európsky významné biotopy na Slovensku. Štátna ochrana prírody SR, DAPHNE - Inštitút aplikovanej ekológie.

Vigo, J. (1974). A propos des forêts de conifêres calcicoles des Pyrénées orientales. Documents phytosociologiques, 7-8, 51-54.

Vigo, J. (1979). Les forêts de conifêres des Pyrénées catalanes: Essai de revision phytocenologique. Documents phytosociologiques N. S., 4 , 929-941.

Voško, M., Kukla, J., Klubica, D., \& Bublinec, E. (1990). Analýza ekologických faktorov a štruktúry lesných ekosystémov monitorovacích plôch. Zborník prác o Tatranskom národnom parku, 30: 227-275.

Vukelić, J., Alegro, A., \& Šegota, V. (2010). Altimontanska-subalpska smrekova šuma s obrubljenim gladcem (Laserpitio krapfi-Piceetum abietis ass. nova) na sjevernom Velebitu (Hrvatska). Sumarski list, 134(5-6), 211-226.

Willner, W. (2007). Nomenklatorischer Anhang. In W. Willner, G. Grabherr (Eds.), A. Drescher, Ch. Eichberger, A. Exner, R. F. Wilfried, S. Grabner, P. Heiselmayer, P. Karner, F. Starlinger, N. Sauberer, \& G. M. Steiner, Die Wälder und Gebüsche Österreichs: Ein Bestimmungswerk mit Tabellen. 1 Textband (pp. 219-245). Elsevier.

Willner, W., Grabherr, G. (eds), Drescher, A., Eichberger, Ch., Exner, A., Wilfried, R. F., Grabner, S., Heiselmayer, P., Karner, P., Starlinger, F., Sauberer, N., \& Steiner, G. M. (2007). Die Wälder und Gebüsche Österreichs: Ein Bestimmungswerk mit Tabellen. 2 Tabellenband. Elsevier.

Willner, W., Theurillat, J.-P., Pallas, J., \& Mucina, L. (2015). On the nomenclature of some high-rank syntaxa of European forest vegetation. Phytocoenologia, 45(1-2), 175-181. https://doi. org/10.1127/phyto/2015/0036

Wraber, M. (1964). Eine neue Fichtenwaldgesellschaft am Übergang der Ostalpen in das dinarische Gebirge. Acta botanica Croatica, Vol. extraord., 125-132.
Wraber, M. (1969). Subalpinski smrekov gozd na Kočevskem in njegova horološko-ekološka problematika. Varstvo narave, 6, 91-104.

Wraber, M. 1(970). Die obere Wald- und Baumgrenze in den slowenischen Hochgebirgen in ökologischer Betrachtung. Mitteilungen der Ostalpin-dinarischen Gesellschaft für Vegetationskunde, 11, 235-248.

Zahradníková-Rošetzká, K. (1957). Príspevok ku kvetene Demänovskej doliny. Biologické práce, 3(4), 1-60.

Zięba, A., Różański, W., \& Szwagrzyk, J. (2018). Syntaxononomy of relic Swiss stone pine (Pinus cembra) forests in the Tatra Mountains. Tuexenia, 38, 155-176. https://doi.org/10.14471/2018.38.004

Zięba, A., Różański, W., Bukowski, M., Ciesielska, B., \& Szwagrzyk, J. (2019). Distribution and habitat conditions of Pinus cembra forests in the Tatra Mountains. Dendrobiology, 81, 86-96. https://doi. org/10.12657/denbio.081.010

Zlatník, A. (1957). Využití generálních typologických map k tvoření územních celků a jejich význam pro lesnickou praxi. Sbornik Vysoké školy zemédèlské a lesnické v Brnè, 1957(2): 75-89.

Zlatník, A. (1959). Přehled slovenských lesů podle skupin lesních typů. Spisy Védecké laboratore biogeocenologie a typologie lesa Lesnické fakulty Vysoké školy zemédělské v Brně, 1959(3), 1-92.

Zlatník, A. (1970). Ekologicko-synekologický, cenologický a fytogeografický výskum na trvalých výskumných plochách. Zborník prác o Tatranskom národnom parku, 12, 79-152.

Zlatník, A. (1975). Ekologie krajiny a geobiocenologie jako védecký podklad ochranu prírody a krajiny. TIS - Svaz pro ochranu prírody a krajiny, Vysoká škola zemědělská v Brně.

Zlatník, A. (1976). Přehled skupin typů geobiocénů původně lesních a křovinných v ČSSR. (Předběžné sdělení). Zprávy Geografického ústavu CSAV, 12(3-4), 55-64.

Zlatník, A. (1978). Lesnická fytocenologie. Státní zemědělské nakladatelství.

Zukrigl, K. (1973). Montane und subalpine waldgesellschaften am Alpenostrand unter mitteleuropäischem, pannonischen und illyrischen Einfluß. Mitteilungen der forstlichen Bundes-versuchsanstalt Wien, 101, $1-387$.

Zupančič, M. (1980). Smrekovi gozdovi v mrazišč h Dinarskega Gorstva Slovenije. Slovenska akademija znanosti in umetnosti. 